University of Louisville

ThinkIR: The University of Louisville's Institutional Repository

$8-2018$

\title{
Transgender and racial identities negotiation processes of transgender Asians, Asian Americans, and Pacific Islanders : a transcendental phenomenological examination.
}

Jayden Thai

University of Louisville

Follow this and additional works at: https://ir.library.louisville.edu/etd

Part of the Counseling Psychology Commons

\section{Recommended Citation}

Thai, Jayden, "Transgender and racial identities negotiation processes of transgender Asians, Asian Americans, and Pacific Islanders : a transcendental phenomenological examination." (2018). Electronic Theses and Dissertations. Paper 3048.

https://doi.org/10.18297/etd/3048

This Doctoral Dissertation is brought to you for free and open access by ThinkIR: The University of Louisville's Institutional Repository. It has been accepted for inclusion in Electronic Theses and Dissertations by an authorized administrator of ThinkIR: The University of Louisville's Institutional Repository. This title appears here courtesy of the author, who has retained all other copyrights. For more information, please contact thinkir@louisville.edu. 


\title{
TRANSGENDER AND RACIAL IDENTITIES NEGOTIATION PROCESSES OF TRANSGENDER ASIANS, ASIAN AMERICANS, AND PACIFIC ISLANDERS: A TRANSCENDENTAL PHENOMENOLOGICAL EXAMINATION
}

\author{
By \\ Jayden Thai \\ B.A., University of Louisville, 2010 \\ M.Ed., University of Louisville, 2012 \\ A Dissertation \\ Submitted to the Faculty of the \\ College of Education and Human Development \\ in Partial Fulfillment of the Requirements \\ for the Degree of \\ Doctor of Philosophy \\ in Counseling and Personnel Services
Department of Counseling and Human Development University of Louisville
Louisville, Kentucky

August, 2018 
Copyright 2018 by Jayden Thai

All rights reserved 



\title{
TRANSGENDER AND RACIAL IDENTITIES NEGOTIATION PROCESSES OF TRANSGENDER ASIANS, ASIAN AMERICANS, AND PACIFIC ISLANDERS: A TRANSCENDENTAL PHENOMENOLOGICAL EXAMINATION
}

\author{
By \\ Jayden Thai \\ B.A., University of Louisville, 2010 \\ M.Ed., University of Louisville, 2012 \\ A Dissertation Approved on
}

June 28, 2018

by the following Dissertation Committee:

Laurie "Lali" D. McCubbin, Ph.D.: Dissertation Chair

Stephanie L. Budge, Ph.D.

Ahmad R. Washington, Ph.D.

Patrick Pössel, Dr. rer. soc. 


\section{DEDICATION}

This dissertation is dedicated to my incredible parents, brother, family, and ancestors

for your innumerable and unimaginable sacrifices

that gave me the opportunity to dream

and the strength to seek it out. 


\section{ACKNOWLEDGEMENTS}

Dr. Stephanie Budge, thank you for your guidance and mentorship all these years. If it wasn't for you allowing me to be a part of your research lab, I would not be here today. To you, I owe my career. You have guided, nurtured, and supported me throughout my development, not only as a researcher and a psychologist, but also as my authentic self.

Dr. Lali McCubbin, from the bottom of my heart, thank you for taking me under your wings and into your mentorship, supportively pushing me out of my comfort zone, and believing in me when I couldn't believe in myself. You helped me find confidence in not only as a student and a psychologist, you helped me find confidence in myself.

My dissertation committee and auditors, thank you for your patience and energy that allowed me to complete this beast of a dissertation.

Jody Zhong and Ja'lessa Hardwick, thank you for helping me transcribe my interviews. This would've been a much longer and more arduous endeavor without your help.

Mom, Dad, and Steven, you allowed me to follow my dreams and supported me at every step. You have sacrificed so much along the way and those sacrifices have not gone unnoticed. Your love, strength, and encouragements allowed me to flourish. Thank you. I love you.

My chosen family, Dr. Kinton Rossman, Clare Gervasi, and the entirety of the TSTAR Lab. You have been my community and my chosen family. You helped lift me up when life was at its hardest. You gave me a seat at the table (with a bottle of Ale 8) and loved me for who I am. I am forever grateful for your love and friendships.

Luna and Stella, my loves/companions, thank you for your love and emotional support.

Erin Frawley, thank you for your love, support, patience, and tenderness this past year. You have shown me what it means to be truly vulnerable and to be seen in all that I am.

Coffee, you were with me every step of the way.

My participants, thank you for entrusting me with your narratives.

Trans API people everywhere, thank you for being my community. Thank you for being my home. Let us continue to resist, exist, and thrive. 


\begin{abstract}
TRANSGENDER AND RACIAL IDENTITIES NEGOTIATION PROCESSES OF TRANSGENDER ASIANS, ASIAN AMERICANS, AND PACIFIC ISLANDERS: A TRANSCENDENTAL PHENOMENOLOGICAL EXAMINATION Jayden Thai
\end{abstract} June 28, 2018

While the field of transgender (trans) research is vastly growing and expanding, little is known about the process in which trans Asian and Pacific Islanders (API) individuals experience, navigate, and negotiate their trans and API cultural identities. The aims of this study were to: 1) examine the navigation and negotiation process for trans API individuals related to their cultural identities and values; 2) enhance, enrich, and diversify existing trans and API literature by generating foundational and complex understanding of trans API identity processes; and 3) challenge hegemonic narratives regarding trans API individuals' lived experiences. Moustakas' (1994) transcendental phenomenological qualitative methodology was employed to achieve these objectives. Semi-structured interviews were conducted via Skype with a sample of ten trans API individuals, with each interview transcribed, coded, and analyzed according to the methodology. The data yielded eight themes related to the navigation and negotiation process for this population: Navigating Belongingness (\& Lack Thereof), American Cultural Values, API Cultural Values, Cultural Influences on Gender Identity, Hesitancy, 
Coming Out, Personal Values, and Integration. Findings revealed participants' experiences of navigating spaces and negotiating cultural identities/values was far from monolithic and unidimensional. Instead, they spanned across wide spectrums of emotions and understandings of their lived experiences. Their identity processes of navigation and negotiation challenges the clinical implications and Western conceptualization of self-determination. For these participants, self-determination emerged in a way that considered their cultural values and the meaning they made from their experiences. 


\section{TABLE OF CONTENTS}

\section{PAGE}

DEDICATION

ACKNOWLEDGEMENTS

iii

ABSTRACT

LIST OF TABLES

iv

$\mathrm{V}$

ix

CHAPTER 1: INTRODUCTION 1

CHAPTER 2: LITERATURE REVIEW

Identity Navigation and Negotiation 7

API Culture and Community 11

Experiences of Lesbian, Gay, Bisexual, and Queer API Individuals 26

Experiences of the Trans Community in the U.S. 35

Experiences of Trans API People $\quad 47$

Literature Methodology Overview and Limitations $\quad 52$

$\begin{array}{ll}\text { Summary } & 56\end{array}$

$\begin{array}{ll}\text { Present Study } & 57\end{array}$

CHAPTER 3: METHODOLOGY 59

$\begin{array}{lr}\text { Sampling } & 59\end{array}$

$\begin{array}{ll}\text { Participants } & 60\end{array}$

Research Protocol 63

$\begin{array}{lr}\text { Procedure } & 63\end{array}$ 
$\begin{array}{ll}\text { Analytic Method } & 64\end{array}$

$\begin{array}{lr}\text { Researcher and Bracketing } & 69\end{array}$

CHAPTER 4: RESULTS 72

Theme: Navigating Belongingness (\& Lack Thereof) 72

Theme: American Cultural Values $\quad 89$

$\begin{array}{ll}\text { Theme: API Cultural Values } & 91\end{array}$

Theme: Cultural Influences on Gender Identity 96

$\begin{array}{lr}\text { Theme: Hesitancy } & 104\end{array}$

$\begin{array}{lr}\text { Theme: Coming Out } & 110\end{array}$

$\begin{array}{ll}\text { Theme: Personal Values } & 114\end{array}$

$\begin{array}{lr}\text { Theme: Integration } & 119\end{array}$

$\begin{array}{ll}\text { CHAPTER 5: DISCUSSION } & 134\end{array}$

Composite Textural Description: The "What" 135

Composite Structural Description: The "How" 143

$\begin{array}{ll}\text { Textural-Structural Synthesis: The Essence } & 145\end{array}$

$\begin{array}{lr}\text { Clinical Implications } & 150\end{array}$

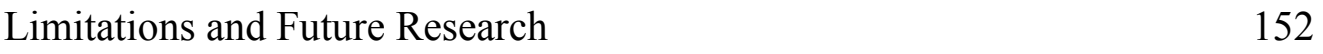

$\begin{array}{ll}\text { REFERENCES } & 156\end{array}$

$\begin{array}{ll}\text { APPENDICES } & 185\end{array}$

$\begin{array}{lr}\text { Appendix A } & 185\end{array}$

$\begin{array}{lr}\text { Appendix B } & 188\end{array}$

$\begin{array}{ll}\text { CURRICULUM VITA } & 189\end{array}$ 


\section{LIST OF TABLES}

TABLE

PAGE

1. Interpersonal and Systemic Anti-Trans Microaggressions

2. Participant Demographic Information 61

3. Theme: Navigating Belongingness (\& Lack Thereof) 73

4. Theme: American Cultural Values $\quad 90$

5. Theme: API Cultural Values 92

6. Theme: Cultural Influences on Gender Identity 96

$\begin{array}{ll}\text { 7. Theme: Hesitancy } & 104\end{array}$

8. Theme: Coming Out 111

9. Theme: Personal Values 115

$\begin{array}{ll}\text { 10. Theme: Integration } & 119\end{array}$ 


\section{CHAPTER I}

\section{INTRODUCTION}

The Asian, Asian American, and Pacific Islander (API) population is one of the fastest growing groups of people in the United States of America and, as of 2010, the API population makes up almost $6 \%$ of the total U.S. population (Hoeffel, Rostogi, Kim, \& Hasan, 2012). The API population comprises of a multitude of diverse ethnicities, though they are often aggregated under one large API umbrella. API ethnicities are typically grouped by regions, for example: East Asia (e.g., China, Japan, Korea, Taiwan), Southeast Asia (e.g., Cambodia, Indonesia, Laos, Malaysia, Myanmar, Singapore, Thailand, Vietnam), South Asia (e.g., India, Nepal, Pakistan), and Pacific Islands (e.g., Hawaii, Samoa, Tonga, Guam). For the purpose of simplicity, API ethnicities will be aggregated under one API definition, though differences in API ethnicities may be described in various bodies of literature that examines only one or a few specific API ethnicities.

Differences in generations (first generation, second generation, etc.) influence the acculturation/enculturation process for individuals (e.g., Koh, Shao, \& Wang, 2009). Immigration patterns contribute to the generational differences that are often observed among API communities. For example, Vietnamese, Hmong, and Laotian communities may only have first (immigrants and refugees of the new country) and second (children of immigrants and refugees born in the new country) generation families due to this population immigrating to the U.S. in more recent history. However, Chinese and 
Japanese people first immigrated to the U.S. in the late 1800s during the country's railroad development; thus, it is not uncommon for these ethnic communities to have fourth and/or fifth generation families (Hune, 2002). Additionally, Pacific Islanders' history with the U.S. may involve colonialization and/or involuntarily annexation of military occupied zones, which inherently affects the relationship Pacific Islanders have with the U.S. and how these relationships change over time and generations. Acculturation — the process of adapting to a new, dominant culture—affects API individuals' experiences and how they relate to their environments, particularly to the individualistic U.S. society (Kim \& Abreu, 2001; LaFromboise, Cole, \& Gerton, 1993). For instance, despite variations in generations and acculturation between API community members, collectivistic API cultural values are often still maintained among API communities due to cultural values acculturating at a different, and often much slower, rate than cultural behaviors (Bacallao \& Smokowski, 2009; Kim \& Omizo, 2006; Marín \& Gamba, 2003; Ryder, Alden, Paulhus, 2000; Szapocznik, Scopetta, Kurtines, \& Aranalde, 1978). The conflict between individualism and collectivism within an individual has the potential to create interpersonal and intrapsychic difficulties (Shin, 1994). This may be apparent in the lived experiences of API individuals who live in both individualistic and collectivistic cultural spaces, such as trans (people who identify with the gender[s] different from their sex assigned at birth, including those who do not identify on the binary gender spectrum) API individuals. Trans API individuals may have to negotiate between their trans identity (influenced by U.S. individualistic cultural values) and their API identity (influenced by API collectivistic cultural values). In addition to balancing cultural values and spaces, trans API individuals also encounter 
double discrimination from their communities such as racism from their predominantly White trans and queer communities and transphobia from their API families and communities. Additionally, these trans and queer communities are informed by the White gaze.

Cisgender (people who identity with the same gender as the one they were assigned at birth) lesbian, gay, bisexual, and queer (LGBQ) API individuals experience similar struggles of navigating and negotiating between cultures, as well as experiencing racism and homophobia/queerphobia from both communities (e.g., Choudhury et al., 2005; Dang \& Hu, 2005; Nadal \& Corpus, 2013). Because LGBQ identities are invisible identities, LGBQ API individuals often have to examine the advantages and disadvantages of coming out to their families as LGBQ (e.g., Mao McCormick, \& de Ven, 2002), or determine if they should live a double life where they are out as LGBQ to their friends but stay closeted with their families (e.g., Chung, Oswald, \& Wiley, 2006). However, trans API individuals have an additional minority identity that goes against traditional API gender norms and expectations. Unlike their LGBQ API peers who are able to keep their LGBQ identities hidden, trans API individuals are not able to hide their trans identities from their API families and communities because of the visible, and often physical nature, of trans identities. Thus, living a double life may not be possible for this population.

There is some literature that focuses on the experiences of trans API individuals, but most of this work is based on populations of trans API people growing up and living outside of the U.S. (e.g., Bakla in the Philippines, Hijra in India, Fa'afaine in Samoa, Kathoey and Phuying Praphet Song in Thailand, etc.) and in various countries in Asia 
and the Pacific Islands (e.g., Kalra, 2012; Vanderlann, Petterson, Mallard, \& Vasey, 2015; Winter, 2006; Winter, Rogando-Sasot, \& King, 2007). While these studies represent important contributions for the overall trans field of research, particularly the trans API literature, they may be limited in drawing attention to the complex, and potentially conflicting, experiences of trans API individuals living in the U.S. and experiencing the intersectionality of their identities with U.S. culture.

Though the literature on trans API individuals in the U.S. is an emerging area of research, there remains some methodological and conceptual shortcomings. For instance, much of the literature related to trans API individuals either conflate trans API individuals' experiences with the experiences of White trans people, trans people of color, or LGBQ API individuals, or the sample size of trans API individuals are minuscule and non-representative of the community (e.g., Dietert \& Denice, 2013; Poteat, German, \& Kerrigan, 2013; Singh, 2013; Singh \& McKleroy, 2011; White \& Ettner, 2014). The majority of the research methodologies (e.g., sampling) do not allow for the examination of trans API populations. Filling in the gap in the trans API literature requires comprehensive understanding of their experiences. Particularly, this includes their experiences with negotiating their multiple cultural identities into one sense of self and navigating cultural environments. In the context of this study, identity negotiation describes the dynamic intrapsychic process of integrating and intertwining interculturalspecific knowledge, expectations, and values to formulate a personal identity of the core self. Navigation is defined as the process of interpersonally communicating with others and interacting with intergroup social surroundings while holding their personal identities and sociocultural group memberships. 
The negotiation and navigation process of balancing cultural values and identities in multiple contexts may take various forms. For example, trans API individuals may have to negotiate their identities and values to decide whether to hide their identities and live in the closet, or to live their authentic lives and risk discrimination from their families and communities in navigating their cultures. The psychological impact of such a process could be better understood with having a foundational understanding of their internal processes or struggles with their cultural values. With limited research about this process and phenomenon, specifically related to American trans API populations, such knowledge must be built from the ground up before further theoretical underpinnings and quantitative examinations can begin to be undertaken.

Phenomenological qualitative research is a fitting methodology to begin building such a foundation of knowledge (Creswell, 2013; Moustakas, 1994). Conklin (2007) posits phenomenology as a creator of knowledge, stating "[i]t is, inherently, a means of creating knowledge that is particular-knowledge that offers a portal of insight into the individual and idiosyncratic" (p. 276). In particular, Moustakas' (1994) transcendental phenomenology or psychological phenomenology approach is chosen because it focuses the examination on the "what" and "how" of individuals' common lived experiences. Specifically, this approach will look at the "what" phenomenon of cultural identity negotiation, and the "how" as it is experienced by trans API individuals. Additionally, from a constructivist epistemological stance (the belief that there is no objective truth, but rather, subjectively constructed interpretations of lived experiences), transcendental phenomenologists deliberately strive for phenomenological reduction. Phenomenological reduction calls for the phenomenon to be reduced to purely the subjective experiences of 
the targeted individuals. While subjective, these experiences have concrete implications on individuals and hold its own meanings unique to the individual. Observing these objective personal experiences sheds light on the lived realities of this population. The researcher's own personal experiences, biases, and assumptions of the phenomenon are also required to be removed as much as possible through the process of bracketing (or the concept of epoché), to ensure that the experiences of the phenomenon are the participants' alone.

This dissertation aims to contribute to the overall trans API literature bringing into awareness the phenomenological process of racial/ethnic, gender, and cultural identity negotiation among trans API individuals and how they navigate themselves in various contexts. It attempts to resist and disrupt the racially homogenous trans literature by diversifying its narratives. Additionally, it hopes to illuminate the socio-historicalpolitical considerations influencing the role of race/ethnicity, gender, and culture on trans API individuals' lived experiences. It further strives to honor the narratives and lived experiences of this population by acknowledging the potential existence of inherent social structures (including but not limited to dominant, oppressive, and/or supportive structures) impacting and influencing their realities. 


\section{CHAPTER II}

\section{LITERATURE REVIEW}

This literature review will begin with a brief description and operationalization of identity negotiation and navigation for the purpose of this study. Next, the literature review will describe API cultural considerations, such as API cultural and familial values, family structures, and acculturation and generational differences among API individuals. Next, to better understand the experiences of trans API individuals, literature on lesbian, gay, bisexual, and queer (LGBQ) API individuals will be described; an emphasis will be placed on the experiences of discrimination and acceptance from the LGBQ community as well as from their family and how these experiences have affected this population's mental health. This is followed by a detailed look at the Western conceptualization of gender and trans identity and different experiences of the general trans community in the United States. Together, these will allow readers to have a fuller understanding of the influences and intersectionality of culture, communities, and identities and how these affect the experiences of trans API individuals. Finally, this chapter will conclude by reviewing the limitations of existing literature and research of trans API individuals.

\section{Identity Negotiation and Navigation}

It would be difficult to discuss trans API identities and their identity negotiation process without defining identity. The terms, negotiation and navigation, were built from Ungar's (2005) original works on resilience and applied to identity processes. According 
to identity theory and identity negotiation theory, identity is defined twofold: (1) social identity, the unique ways in which an individual conceptualizes and sees oneself based on the set of culturally-constructed meanings, values, and expectations that are attributed to a role in a sociocultural system in which an individual occupies, and (2) personal identity, the unique attributes of an individual as compared to the attributes of others (Stets \& Serpe, 2013; Stryker \& Burke, 2000; Ting-Toomey, 2005). Through the process of incorporating learned sociocultural influences and personal lived experiences, an identity (or identities) is (are) formed (Burke \& Tully 1977; Stets \& Burke, 2000). Stets and Burke (2000) succinctly describes individuals and their multifaceted identities as the composition "of the self-view that emerge from the reflexive activity of selfcategorization or identification in terms of membership in particular groups or roles" ( $\mathrm{p}$. 225). Identity is further influenced by interpersonal interactions with those within and outside of their group memberships.

Identity negotiation theory posits that individuals encounter processes of communication (verbal and/or nonverbal) with others in such a way that attempts to validating their group memberships (Ting-Toomey, 2005). Ting-Toomey (2005) posits that individuals seek mutual affirmation of their identity in their interpersonal interactions and communications. It is through these interpersonal interactions that individuals attempt to negotiate with each other on two conditions: the meanings of the situation to provide context for their exchanges, and their identities in such a way that tries to maintain their authentic selves (Stets \& Burke, 2000). To achieve competent negotiation of identities is to mutually feel affirmed, valued, respected, and understood, though this process is not always successful (Ting-Toomey, 2005). This process can be observed in 
Hotta and Ting-Toomey's (2013) study. Hotta and Ting-Toomey qualitatively examined the narratives of twenty international students and their adjustment and relationships with their host families. An emerging theme indicated participants felt the need to adjust their identity and shift their communication styles with their host country. Participants recognized the need to change and expressed feeling the need to engage in behaviors that they typically would not do in their home countries as a way to adjust their identity to better fit their host environment. One participant described the need to adjust her reserved personality to become an "Americanized talkative self" to better fit in with the learning style of the American classroom. The researchers also found that many of the participants indicated that they struggled with feeling validated and often felt rejection from their host family due to being perceived as too different. Desiring acceptance and belongingness, some students attempted to navigate these contexts by participating in behaviors that would demonstrate them as "worthy guests." Despite attempting to navigate their environment, some participants continued to struggle with their host country and maintaining authenticity. This process of identity negotiation and contextual navigation stresses interpersonal and environmental interactions and their effects on identity management. Using this conceptual understanding of contextual navigation could be applied to trans API individuals' navigation of various contexts and how this influences their identity management. For the purpose of this study, this process describes the term navigation, such that individuals are navigating their environment based on their identities.

In addition to the interpersonal processes of identity is the intrapsychic (or internal) processes that influence identity development and management. The term 
negotiation, in this study, is operationalized as the intrapsychic (rather than interpersonal) process of integrating, evaluating, and understanding their identity according to their intercultural knowledge, values, and experiences. Personal values, a fundamental and central facet of an individual's conceptualization of the self, have cultural significances and are developed from sociocultural interactions and contexts (Hitlin, 2003; Schwartz, 1992, 1994). Hitlin (2003) posits that values are interrelated to identity and are key indicators of one's identity. To demonstrate his assertion, he studied the link between values and an identity of being a volunteer. He measured the values and volunteer identity of 314 undergraduate students over the course of their first year of college. Results showed that benevolence and universalism were positive predictors of a volunteer identity. In fact, having greater concern for their self-transcendence values was related to perceiving their volunteer identity with higher importance and identifying as a volunteer with greater strength. Conversely, indication of higher self-enhancement values related to attaining power and/or achievement was less likely to predict a strong volunteer identity. While Hitlin's study only examined the effects of values on a volunteer identity, one could presume a similar effect with other cultural values and their influence on an individual's cultural identity (i.e., trans and API identities) such that the cultural values are key factors and central to an individual's core sense of self.

Identity is developed through the navigation of interpersonal contexts and the negotiation of intrapsychic processes influenced by cultural values and knowledge. Throughout the lifespan, individuals will encounter more interpersonal situations and gain additional cultural experiences. The perpetual changing of the interrelated process of navigation and negotiation causes individuals to continually reevaluate and reconfigure 
their identities over time. Thus, understanding the identity processes of trans API individuals requires a comprehensive understanding of the interpersonal contexts and cultural values and experiences in which their trans and API identities are anchored.

\section{API Culture and Community}

API cultural values. Trans API individuals are not only influenced by their trans identities and the cultural aspects of the trans community but also by their API identities and the traditional API cultural values in which they are raised. To contextualize the experiences of trans API individuals (i.e., how they internally negotiate their trans and API identities, how they externally navigate cultural environments), a review of API cultural values and family/community structures that occupy their API identities is necessary. These traditional API values have been frequently documented in the literature (e.g., Kim, Atkinson, \& Umemoto, 2001; Kim, Yang, Atkinson, Wolfe, \& Hong, 2001). Though, there are fewer studies on API cultural values regarding lesbian, gay, bisexual, queer, and trans API populations. The emphasis on the family and community, as well as an individual's role amongst these structures, have been found to be salient themes of traditional API cultural values.

While Western societies place significant cultural value on individuality, independence, self-determination, autonomy, and uniqueness, Eastern societies, such as API societies, place cultural value on collectiveness, conformity, and harmony of the group and the maintenance of the interpersonal relationships within the group (Triandis, 2001). Recruiting samples from Western and Eastern locations (Illinois, Greece, Hawaii, Hong Kong, China), Triandis, McCusker, and Hui (1990) found collectivists distance themselves from those outside of their groups while closely including themselves with 
those within their groups. Collectivistic identification emphasized integrity within their group rather than hedonism, which was emphasized among individualistic identification (Triandis et al., 1990). Moreover, collectivists defined themselves more in terms of their family and their ethnic group (Triandis et al., 1990). Among many API cultures cultural collectivism stresses the cultural value of community such that:

" $[r]$ ather than an 'I' identity, Asians are taught to embrace a 'we' identity. The individual does not stand alone, but is seen as the product of all the generations of his or her family and is therefore held in relation to them" (Lee \& Mock, 2005, p. 271).

Using a sample of undergraduate students including East Asians, Singelis, Triandis, Bhawuk, and Gelfand (1995) reported that while individualism and collectivism are separate constructs, horizontal and vertical individualism and collectivism are also separate and distinct constructs. According to Singelis and his colleagues (1995), a vertical orientation emphasizes inequality and a horizontal orientation stresses equality. Vertical collectivism is operationalized as perceiving oneself as part of the collective group, though also acknowledging and accepting the existence of hierarchal, or vertical, inequalities among the members of the collective group; on the other hand, horizontal collectivism is defined as perceiving oneself as part of the collective group as well as viewing each member of the group as equals (Singelis et al., 1995). Similarly, vertical individualism is defined as perceiving oneself as autonomous and independent, while accepting inequality exists among individuals; horizontal individualism is operationalized as perceiving oneself as autonomous and independent, but emphasizes equality among individuals. In regards to API participants, Singelis et al. (1995) found a strong positive correlation between API participants and vertical collectivism, indicating that API individuals are not only perceiving themselves as part of their ingroup, but also 
acknowledging unequal power statuses within their ingroup. They also found a negative correlation between vertical collectivism and European Americans, such that European Americans perceives themselves as less a part of their ingroup and less accepting of the hierarchal inequalities within the collective group.

Differences between individualistic and collectivistic values can be observed in their displays of social conformity and avoidance of deviance, such as conflict communication. A social norm for individuals in individualistic cultures is to confront conflicts actively and assertively to determine resolutions; individuals influenced by individualistic cultural values perceive these "conflictual" social interactions as part of the natural flow of life and strive to stand out as a unique individual (Ohbuchi, Fukushima, \& Tedeschi, 1999). Conversely, the social norm for individuals in collectivistic cultures is to work together collaboratively and passively, even use avoidance, to resolve conflicts (Ohbuchi et al., 1999; Ohbuchi \& Takahashi, 1994; Trubisky, Ting-Toomey, \& Lin, 1991). Conformity of social norms is one of the cultural values found in many API cultures (Kim, Atkinson, \& Yang, 1999). Deviance can be understood in relation to norms. Engaging in behaviors that deviates from social norms could result in a loss of face (e.g., loss of integrity), respect, and social statuses (Ho, 1976; Kim et al., 2001). Thus, it is not uncommon for individuals influenced by collectivistic cultural values to avoid losing face for themselves and their families by blending into their social surroundings and abiding by social norms (Morrow, 1987). Kim and Markus (1999) conducted social psychology experiments with Chinese and European Americans for the purpose of examining how individualistic and collectivistic values influence a person's preference for deviance/uniqueness or harmony/conformity. 
They found that Chinese Americans had a preference towards conformity with another individual and avoidance of deviance and uniqueness, even with abstract figures and color pens. In two separate experiments, it was found that when given a choice, Chinese American participants had a preference towards abstract figures and pens that were similar to the others in the group while European American participants had a preference towards figures and pens that were different from the others in the group. For Chinese Americans, conformity and avoidance of deviance/uniqueness appeared to be in accordance to their cultural backgrounds and values, such that one conforms to social/cultural norm and avoids deviating from it. There are clear social expectations and understandings of what behaviors are "normal" and "appropriate;" each individual has a set of clearly defined expectations and roles in the family and community in accordance to their gender, age, and social status (Lee \& Mock, 2005).

Social and cultural expectations inherently influence normative gender roles and allow little deviance from them, such that traditional gender roles are often clearly defined, expected, and enforced for men and women and for each family member (Ho, Rasheed, \& Rasheed, 2004; Sue \& Sue, 1993). These gender roles are also conflated with sex, such that gender is understood to be a biological category. Traditional gender roles in API cultures emphasize a binary ideology of nature and the complementariness of these natural occurrences, which upholds the harmony of humanity. For example, some API cultures believe in the concept of "Yin and Yang," which says that everything in nature has two opposite sides that complement each other; this concept often represents contrasting, yet complementary and unifying ideas such as light and dark, day and night, warmth and cold, wrong and right, and in the context of humans, women and 
men (Chung \& Katayama, 1998). Because these traditional social ideologies of gender strive for the natural and harmonious continuation of the family, and ultimately their community, there are specific heteronormative roles for men and women to fulfill.

As per traditional gender role assignments in East and Southeast Asian cultures, men are expected to grow up to be fathers and continue the family name and lineage; thus, men in these cultures are more respected and regarded as more important and valuable than women (Shon \& Ja, 1982). Men are expected to be the heads of their households (Ho et al., 2004; Lee \& Mock, 2005; Sue \& Sue, 1993). As adults with families of their own, men are regarded as the unchallenged authoritarian figures of their family, with the responsibility of ensuring the family rules and values are stringently enforced to maintain social organization (Lee \& Mock, 2005). They make the decisions for the family and in all family matters to lead the family to success. Traditionally, not only must the oldest son bear the burden of the responsibility of providing for and protecting his entire family, he is also responsible of taking care of his elderly parents and being role models for his younger siblings throughout the duration of his adulthood (Lee \& Mock, 2005; Park \& Chesla, 2007; Sue \& Sue, 1993). As the leader of the family any successes or failures of a specific family member are attributed to the father's abilities to direct and discipline his family. Fathers further assume the responsibility of their community's success because the community's success is predicated on the family's success, their adherence to social norms, and their ability to maintain social order. While men have more outspoken roles in the family and community, women's roles have been traditionally rooted within the home environment (Lee \& Mock, 2005; Sue \& Sue, 1993).

Contrasting to traditional gender roles assigned to men, women are expected to 
grow up and marry a husband, be mothers, and quietly take care of their family and household (Ho et al., 2004; Lee \& Mock, 2005; Sue \& Sue, 1993). The caretaker role consists of managing and maintaining the household, being the homemaker, and providing nurturing physical and emotional support for their family members (Ho et al., 2004; Lee \& Mock, 2005; Shon \& Ja, 1982; Sue \& Sue, 1993). In a sample of Indian mothers in India, Tuli and Chaudhary (2010) found a consistent theme among their participants was the central role of the mother in the mother-child relationship (e.g., Indian mothers determine the level of agency and independence of their children based on different domains). Additionally, consistent with previous API literature on genderspecific roles, Tuli and Chaudhary (2010) found that Indian fathers' roles within the family environment are described as intense and important, but limited in shared parenting and household duties. Because of their nurturing role, mothers tend to have a stronger emotional relationship with the children than do fathers, thus children often go to their mothers first in search for emotional support and have their mothers advocate on their behalf to their fathers. These cultural heteronormative, patriarchal ideologies construct these traditional gender roles as purposeful, necessary, and natural, such that they provide structure for families to thrive with the belief that collectively, thriving families contribute to a thriving community and society.

Deviation from these assigned roles means the digression from nature's harmony, rejection of societal norms, risk of the advancement of the family lineage, the potential betrayal of the community, and the creation of intrapsychic and social conflict, confusion, and disorganization. Among a sample of Indian families, Dasgupta's (1998) study found that not only are the expectations of traditional gender roles pervasive in the community, 
but that the women in the sample felt confined by these expectations. Moreover, Dasgupta (1998) found the women, men, and children of the families had highly similar attitudes towards the role of women in their society. On the contrary, participants of Chinese decent in Tang and Dion's (1999) study indicated that the men held more traditional gender role expectations than the women of the sample, but their expectations were similar to those of their traditional parents. This is similar to the findings of Rosenthal, Ranieri, and Klimidis (1996), which found Vietnamese young adult women who immigrated to Australia held less traditional Vietnamese family values than their male counterparts. Furthermore, these studies revealed an association between perceiving one's parents as traditional and feelings of alienation from their community and society.

Adherence of API cultural values have been measured using the Asian Values Scale (AVS; Kim et al., 1999). The AVS is a well-known instrument that has been cited in the literature well over five hundred times and been used in studies to look at a wide range of API experiences, such as intergenerational familial conflict (e.g., Park, Kim, Chiang, \& Ju, 2010; Tsai-Chae \& Nagata, 2008), acculturation (e.g., Shim \& Schwartz, 2008), cultural-specific emotion regulation (e.g., Butler, Lee, \& Gross, 2007; Iwamoto, Liao, \& Liu, 2010), and help-seeking attitudes (e.g., Kim \& Omizo, 2003; Zhang \& Dixon, 2003). Kim and colleagues (1999) conducted an in-depth review of the literature by API acculturation and cultural value scholars to generate the ten dimensions of API cultural values. Exploratory factor analysis confirmed six factors of API cultural values: conformity to norms, family recognition through achievement, emotional self-control, collectivism, humility, and filial piety. Generational differences and 
acculturation/assimilation also affects the salience of the cultural values; however, this will be discussed in further detail later. However, post-colonial gender roles of API cultures, while are not all the same, endorse strict binary male-female gender expectations, which erases, minimizes, or pathologizes non-binary gender identities which previously existed in various API cultures; this will also be described in detail later.

Family Values. Traditionally, the family is the central institution of API culture and the centerpiece of the community. The community structure is often viewed as being constructed of family units rather than of individual members. As described by Park and Chesla (2007), “[B]oth community and society are a mere extension of the family" (p. 305). Any breakdown of the family structure could lead to the breakdown of the community. Thus, the cultural standards and expectations of acceptable behaviors and values are dictated and enforced by the family in order to maintain harmony of the family unit and social organization of the whole community (Park \& Chesla, 2007).

The collectivistic social structure of the family influences the individual lives within these cultures, such that the decisions, behaviors, and values of individuals and their conceptualizations of themselves are intrinsically interwoven within the family and community structure. For example, traditional familial values require that the needs of the family supersede the needs or desires of its individual members (Choudhury et al., 2009). Younger individuals are expected to show absolute, unquestioning reverence, respect, and obedience for their elders, regardless of their own personal beliefs (known as filial piety; Kim et al., 2001; Kim \& Wong, 2002; Lee \& Mock, 2005). Filial piety also holds the expectation that adult children will make personal sacrifices to take care of their 
parents, especially as their parents age and become physically incapable of taking care of themselves (Kim et al., 2001; Kim \& Wong, 2002).

Attempts have been made to operationalize the Confucius philosophy and teaching of filial piety, though it is cannot be easily operationalized quantitatively (Liu, Ng, Weatherall, \& Loong, 2000). Jones, Lee, and Zhang (2011) identified three factors related to filial piety (responsibility, respect, and care) across five different cultures (Asian, African, European, Latino, and Native American), while Lum et al. (2015) identified two factors (pragmatic obligations and compassionate reverence). Similarly, literature on family obligations have quantitatively emerged as a concept related to filial piety. Fuligni, Tseng, and Lam (1999) examined family obligations by measuring the extent of current familial assistance, one's respect for family, and the expectation of future familial support. They found Asian American adolescents to endorse stronger values and expectations regarding family obligations than their White counterparts. Furthermore, Kim and Wong's (2002) review of the literature on anthropological fieldwork using qualitative approaches and direct observations of indigenous API communities revealed themes of filial piety and family structure. Thus, it is unsurprising that API individuals often adjust their individual needs to align with the needs of their family and adhere to cultural and familial standards to maintain family harmony (Sue \& Sue, 2008). As such, API cultural values emphasize a strong devotion and loyalty to the family unit.

Loyalty to the family unit implies the protection of the family integrity or "saving face." From a sociological perspective, face can be defined as an individual's symbolic, projected sense of identity, integrity, dignity, worth, and respect they exude through 
social interactions to maintain their self-presentation and social statuses relative to their social systems (Ho, 1976; Ting-Toomey, 1994). As described by Goffman (1955), face is "the positive social value a person effectively claims for himself...during a particular contact. Face is an image of self delineated in terms of approved social attributes" (p.

213). Ho (1976) provided an advanced definition of the complexity of face:

Face is the respectability and/or deference which a person can claim for himself from others, by virtue of the relative position he occupies in his social network and the degree to which he is judged to have functioned adequately in that position as well as acceptably in his general conduct; the face extended to a person by others is a function of the degree of congruence between judgments of his total condition in life, including his actions as well as those of people closely associated with him, and the social expectations that others have placed upon him. In terms of two interacting parties, face is the reciprocated compliance, respect, and/or deference that each party expects form, and extends to, the other party (p. $883)$.

While face is not a concept exclusively found in API cultures the literature, scholars have consistently determined it to be an important concern in API cultures (Ho, 1976; Kim \& Nam, 1998; Mak \& Chen, 2006). The loss of face is due to an individual's unacceptable or unsatisfactory behavior, performance, or conduct. In addition to the loss of the aforementioned identity, integrity, dignity, worth, and respect, losing face refers to the loss of social status within the community and social networks. Protection and maintenance of one's face is of utmost importance "because of the demoralizing repercussions which otherwise follow" (Ho, 1976, p. 871). A loss of face not only affects the individual's social standing it also affects their family's reputation and social standing. Bringing shame onto the family and family members through one's own unacceptable behaviors defies the virtue of filial piety. Thus, it is expected that individuals may make personal sacrifices to maintain their family's face for the greater 
good of their family and community. For example, in order to save face for themselves and their families, API individuals may refrain from seeking or underutilize mental health services due to the taboo nature of mental illnesses in API cultures; rather, the more socially acceptable alternative is turning to families for support or deal with their struggles on their own (Kim \& Omizo, 2003; Leong \& Lau, 2001). Furthermore, an API individual's self-worth has been described as being tied to their family's face (Sue \& Sue, 1993).

To preserve social harmony, traditional API cultural values emphasize community and family as central social structures in one's identity. It is unsurprising that individuals may experience an instinctual imperative to adhere to culturally normative values to maintain close social connectedness and belongingness with their community and family (Asakawa \& Csikszentmihalyi, 2000; Markus \& Kitayama, 1991). Adherence to these cultural and familial ideologies may vary depending on the API individual's acculturation/assimilation to the majority culture, as well as their generational status (Lee $\&$ Mock, 2005; Sue \& Sue, 2003).

Acculturation and Generational Factors. Redfield, Linton, and Herskovits (1936) defined acculturation as a process that "comprehends those phenomena which result when groups of individuals having different cultures come into continuous firsthand contact with subsequent changes in the original culture patterns of either or both groups" (p. 149). Kim and Abreu's (2001) more recent definition of acculturation describes this as the process of an individual adapting their culture of origin to their new, dominant culture; conversely, the process of enculturation is the process and extent to which the individual retains their culture of origin. However, it was Berry (1980) who 
developed a model to describe the process of acculturation and enculturation; he posited four experiences of acculturation: separation, marginalization, assimilation, and integration.

According to Berry's $(1980 ; 1997)$ acculturation model, separation is the process in which an individual strives to hold on to their culture of origin, while separating themselves from the new, dominant culture. Assimilation is the process where the individual strives to fully integrate into the norms of the dominant culture and separate themselves from their culture of origin. Individuals engaged in the marginalization process have little interest in participating in the dominant culture and little interest in maintaining their culture of origin, but does so to keep from completely losing their culture. Lastly, individuals navigating the integration process are able to maintain a degree of connection with their culture of origin, while also interacting and participating in the dominant culture. Individuals attempting to integrate cultural identities may experience varying levels of bicultural stress due to having to navigate and negotiate two different cultures - the pressures of cultural maintenance and the pressures of adapting to the dominant culture (LaFromboise et al., 1993; Romero, Carvajal, Valle, \& Orduña, 2007; Yee, DeBaryshe, Yuen, Kim, \& McCubbin, 2007). Similarly, the process of trying to acculturate into a new, dominant culture can also be stressful (Meyer, Dhindsa, \& Zane, 2011).

While acculturation research continues to disagree on the operationalization of measuring acculturation (Hwang \& Ting, 2008), measurement themes including language competency, social interpersonal relationships, cultural participation, experiences of discrimination, the importance of maintaining and honoring one's traditional cultural 
values, and having a sense of belonging have been included in studies attempting to operationalize acculturation (Salant \& Lauderdale, 2003). Acculturative stress has been measured as the stress and difficulties experienced due to having difficulties with language, instances of discrimination and prejudice, conflicts between opposing cultural values, and feelings of loneliness (Salant \& Lauderdale, 2003; Shin, 1994). The acculturative stress of navigating the dominant culture has been shown to be associated with mental well-being. For example, Romero and colleagues (2007) found that bicultural stress had a significant, positive relationship with depressive symptoms among API and Latino/a participants; when sociodemographic variables (e.g., age, gender, generational factors, socioeconomic status, etc.) were controlled, only the positive relationship between bicultural stress and depressive symptoms among API participants remained compared to European Americans. Among a sample of API participants Hwang and Ting (2003) found that successful navigation within the dominant culture is associated with psychological well-being, regardless of the degree of identification with their culture of origin. Their finding supports previous literature, such that integration (e.g., navigation) of both the culture of origin and the dominant culture is associated with better psychological well-being (e.g., Ryder et al., 2000). Furthermore, not only does acculturation and bicultural stress impact the psychological health of API individuals, acculturative stress has also been found to affect API elders such that the stress from the perceived cultural gap between themselves and their children was associated with high levels of depression (Lee \& Mjelde-Mossey, 2004; Mui \& Kang, 2006).

Acculturation, enculturation, and experiences of acculturative stress vary due to generational differences, also influenced by historical and sociopolitical contexts. For 
example, due to the Vietnam War in the 1960s and 1970s, Southeast Asian Americans are more likely to be first and second generation individuals while other API nationalities are more likely to have older generations (e.g., Chinese Americans can be fourth or fifth generations who can date their American histories back to the first transcontinental railroad or they can be first generation immigrants; Hune, 2002). First and 1.5 generation API individuals (i.e., immigrant and refugee adults and children) may experience acculturative stress due to attempting to uphold the cultural values of their culture of origin (Koh et al., 2009), while second generation API individuals (individuals born to first generation individuals) and above may experience acculturative stress where they struggle to determine which culture to participate in more and how to balance their different (sometimes conflicting) cultural backgrounds.

To add to the complexity of generational differences, Kim et al. (1999) posited that there are two independent constructs to acculturation-behavior and values. Based on the six dimensions of API cultural values of the AVS, they found that generational status only explained a small portion of the variance in adherence to acculturative values but explained $30.4 \%$ of the variance in adherence to acculturative behavior. Ishikawa (2013) found that there were no generational differences in acculturated values between second, third, and fourth generation Japanese Americans, but there were generational differences in acculturated behaviors. Acculturation scholars have posited that acculturation pertaining to values is a much slower process than behavioral acculturation (Szapocznik et al., 1978). This may be due to the survival nature of behaviorally navigating through the dominant culture, such that one blends into the dominant culture rather than stand out and risk being targeted as othered. Successful behavioral navigation 
of the dominant culture (behavioral acculturation) is related to positive psychological well-being. For example, behavioral acculturation has been found to be positively related to mental health benefits, such as general self-efficacy and cognitive functioning, among API college students (Kim \& Omizo, 2006). Even though later generations display more acculturated behaviors, their values remain similar to those of the earlier generations. In other words, individuals hold on to the values of their culture of origin across generations. This has been reported by Kwak and Berry (2001), where it was found that API adolescent immigrants shared the same cultural values regarding family obligations as their parents and that family decisions continue to be made within the family's hierarchical structure. This may cause internal conflict for the individual in the form of bicultural stress, as well as external conflict from family members because of the differences in values and behavioral acculturation processes.

These acculturation gaps among family members can cause familial conflict (Pyke, 2005; Sue \& Sue, 2003; Xia, Do, \& Xie, 2013). The Asian American Family Conflicts Scale (FCS; Lee, Choe, Kim, \& Ngo, 2000) has been used to measure these intergenerational family conflicts. While some studies suggest generational differences can cause external conflict among family members, Kwak and Berry (2001) found family relationships remained cohesive and cooperative despite general differences. The process of acculturation and enculturation, and the consideration of generational factors related to cultural values are relevant in the discussion of API individuals' experiences in the United States, especially of trans API individuals. Fortunately, the literature on acculturation and cultural values among API individuals have been thoroughly and empirically investigated and detailed. The expansive field of study allows for more 
comprehensive understanding of the overall process among API populations. However, these studies continue to lack narratives of trans API experiences.

\section{Experiences of Lesbian, Gay, Bisexual, and Queer API Individuals}

In order to fully understand and appreciate the experiences of trans API

individuals, it is necessary to understand the experiences of lesbian, gay, bisexual, and queer (LGBQ) API people due to their inherent transgressions of heteronormative gender norms. Generally, LGBQ people of color encounter a variety of discrimination from multiple life domains because of their multiple identities. In addition to their general life stressors, LGBQ people of color often experience instances of racism and homophobia. Racism and homophobia manifest themselves among the LGBQ community as well as the racial and ethnic communities with which they identify. Therefore, LGBQ people of color experience racism among the general LGBQ community in addition to the experiences of homophobia from their family and communities of color. Racism and homophobia places LGBQ people of color in positions where they have to navigate multiple stressors of discrimination and prejudice from the communities in which their identities are anchored. In some instances, individuals feel the pressure that they must choose one identity over another - between being a part of their racial/ethnic community or the LGBQ community (Harper, Jernewall, \& Zea, 2004). Among a sample of Latino gay men, Zea, Reisen, and Diaz (2003) found that participants openly identify as gay when they are in the context of gay spaces (e.g., gay bars), but not when they are with their families or in Latino community spaces. Similarly, Tremble, Schneider, and Appathurai (1989) revealed LGBQ youths of color would often avoid participating in familial and cultural activities in fear of bringing shame to their families. 
Experiences of racism. In the field of psychology, empirical investigations into the experiences of racism among LGBQ people of color have been primarily studied through the lens of their multiple intersecting minority identities (e.g., Bowleg, Huang, Brooks, Black, \& Burkholder, 2003; Harper et al., 2004; Hayes, Chun-Kennedy, Edens, \& Locke, 2011). For example, Bowleg and colleagues (2003) conducted a qualitative study of Black lesbian women $(N=19)$ and found their participants encountered "triple jeopardy" (e.g., experiences of racism, sexism, and heterosexism). Their participants reported the common theme of racism as a significant stressor; experiences of sexism and heterosexism were further contextualized in relations to race and racism (e.g., decision to not come out as lesbian due to already being a Black woman in a predominantly White workspace). However, the authors did not specify the source of the racism (e.g., from White people, non-Black people of color, institutional, etc.) that was encountered by their participants. The experiences of racism within the general LGBQ community towards their members of color have been examined and documented, though infrequently. Documentation of the experiences among LGBQ people of color in the literature have historically focused on general anti-LGBQ discrimination and/or their experiences of racism from straight people, rather than the racism that manifests within its own LGBQ community. This may be due to the fact LGBQ literature consists primarily of White participants (DeBlaere et al., 2010; Singh \& Shelton, 2011). Greene (2000) and Harper et al. (2004) cautioned against making generalizations about LGBQ people of color based on research findings that consists primarily of White LGBQ participants. The racial and ethnic imbalance in sampling and topic in the LGBQ field of study has led to Moradi, DeBlaere, and Huang (2010) calling for the intentional centralization of LGBQ people of 
color's experiences in the counseling psychology literature, such that their experiences be more of a focal point of the LGBQ research in the field of counseling psychology. While often overlooked, LGBQ API individuals' experiences of racism from not only the general population, but the LGBQ community, is evident and worthy of investigation.

Contrary to the popular rhetoric of the model minority myth (the distorted, stereotypical notion that all API people are able to achieve unparalleled success and do not encounter hardships because of their race/ethnicity), API people do experience racism and its various discriminatory forms. Similar to LGBQ non-API people of color, LGBQ API individuals also encounter a variety of discriminatory acts due to their multiple identities. In addition to being people of color and sexual minorities, they are specifically API, which has its own socio-political-historical contexts attached to their racial/ethnic identities. Consequently, this influences the intersecting experiences of API identities with LGBQ identities (e.g., cultural values and expectations) and instances of discrimination.

Nadal et al. (2015) utilized qualitative secondary analysis and consensual qualitative research methods to examine the intersectional microaggressions of people with multiple identities. One of the seven themes that arose from their findings was the Exoticization of Women of Color, where subthemes indicated straight, lesbian, bisexual, and queer API women reported being perceived as sexual objects (e.g., "Asian Fetish," "mail-order-brides") or "damsels in distress" waiting to be saved by White men. Conversely, another theme, Invisibility and Desexualization of Asian American Men, illustrated the isolation and rejection both straight and LGBTQ API men experience due to the negative stereotypes associated with API men. Two gay API male participants in 
the study reported being rejected by potential partners on the basis of their ethnicities. Previous literature supports Nadal et al.'s (2015) findings, which further describes how gay API male participants were rejected, isolated, and discriminated against by other men in the gay community, particularly by White gay men (e.g., Chung \& Szymanski, 2006; Han, 2010; Mao et al., 2002; Nadal \& Corpus, 2013). Similarly, lesbian and bisexual API women in Sung, Szymanski, and Henrichs-Beck's (2015) study reported struggling with invisibility, isolation, negation, and lack of representation within the LGBQ community; these participants attributed their challenges to the racism, "whiteness," and "anti-Asian/white supremacist racism" that exists within the LGB community. Supporting the findings of Nadal et al. (2015), Sung et al. (2015) found their lesbian and bisexual API women also experience negative discriminatory sexual encounters, such as stereotypes, fantasies, objectifications, and fetishizations. Furthermore, Choudhury et al. (2009) found 56\% of South Asian American respondents experienced racism, exoticism, and xenophobia from the LGBTQ community. Similarly, Dang and Hu (2005) surveyed 124 lesbian, gay, bisexual, transgender, and queer (LGBTQ) API individuals and found almost every participant (95\%) indicated that they have experienced at least one form of discrimination during their lifetime. Participants overwhelmingly reported experiencing racism within the White LGBTQ community, with $84 \%$ of participants agreeing and $36 \%$ strongly agreeing to experiencing racism from the White LGBTQ community (Dang \& $\mathrm{Hu}, 2005)$. In the same study, $68 \%$ of LGBTQ API participants also agreed that they have experienced racism from other LGBTQ people of color. Even among their own LGBQ community, LGBQ API individuals are not exempt from discrimination and isolation. Unfortunately, LGBQ API individuals not only experience discrimination from 
their LGBQ communities, they also experience discrimination from their API families and communities.

Experiences of homophobia. As Western societies colonized Asia and Pacific Island countries over time, they brought with them homophobic, heteronormative ideologies that ultimately affected how API cultures viewed gender and sexuality (Roen, 2001; Sinnott, 2004). Colonialization influenced the internalization of homophobia into the culture, leading to frequent perception of LGBQ identities as "Western diseases" (Blackwood \& Johnson, 2012; Choudhury et al., 2005). Laurent (2005) posited that many API countries had tolerance toward "queer-like" identities, feelings, and behaviors, such that these identities, feelings, and behaviors were deemed appropriate by the social values of the particular cultures. However, as the presence of Western ideologies began to spread throughout API countries during and post-colonialization, so did the negative perceptions, beliefs, policing, and pathologization of queer identities and practices. Conservative API cultures' intolerance of sexual minority identities was frequently reported (Sung et al., 2015). When API cultures are taken out of the post-colonial gaze (viewing the world through the social, cultural, and political ideology produced by those in power as the consequence of colonialism), some cultures had specific identities contextualized and embedded (e.g., bakla in Filipino culture; hijra in South Asian culture; tom and kathoey in Thailand culture) within its respective cultures that transgress the queer-straight sexuality divide (Blackwood \& Johnson, 2012; Nadal \& Corpus, 2013; Sinnott, 2004).

While their sexual identities are marginalized and undervalued within their own API families and communities, LGBQ API individuals also encounter cultural conflicts 
with the traditional heteronormative gender roles that are often expected of them by their families and API communities. Sexual restraint and modesty are valued social and moral conducts in many API cultures, and are often encouraged to avoid tarnishing the family honor or face (Abraham, 1999; Okazaki, 2002). Thus, in API cultures where sexuality and sexual attitudes are already conservative, it can be difficult for LGBQ API individuals to express their sexual identities in ways that are perceived as culturally appropriate or acceptable. In some cases, API individuals may engage in queer practices in private (e.g., secret engagements, paid sex workers), but avoid labeling themselves as so and continue socially appropriate behaviors in public (e.g., participate in traditionally gendered marriages and/or families; Laurent, 2005). The internalized homophobic and heteronormative belief system places LGBQ API individuals in positions where they would have to consider their API cultural values when attempting to navigate their LGBQ identity among their API communities (e.g., cultural considerations, coming out process, homophobia).

When attempting to navigate API spaces with LGBQ identities, one must navigate the boundaries of socially appropriate behavior. Nadal and Corpus (2013) qualitatively examined the experiences of a sample of LGB Filipino Americans $(N=24)$. The authors found a theme concerning Gender Role Norms, such that LGB Filipino American participants were subjected to stringently defined gender roles by their families. Another theme encountered in the sample was the Unspoken Issues with Sexual Orientation, where participants were unable to openly communicate their sexual identity with their parents; their silence regarding their sexual identity avoids creating conflict among their families. Additionally, Filial Piety was found to influence participants' decision around 
whether or not to come out to their families about their sexual identity, in fear of bringing shame to the family and jeopardizing their familial connectedness and belongingness. LGBQ API individuals further feel the constant pressure of marriage and familial expectations (Sung et al., 2015). Mao et al. (2002) also found the coming out process was a major issue among gay Asian men participants, such that participants reported having to consider and weigh the advantages and disadvantages of coming out to their families. Coming out as LGBQ increases the likelihood of victimization from mainstream society, as well as discrimination within by their own API communities. Instances of homophobia within API communities are common occurrences for LGBQ API individuals. Seventy percent of South Asian American respondents reported experiencing homophobia from the South Asian community (Choudhury et al., 2005). Similarly, almost all participants (96\%) in Dang and Hu's (2005) study reported homophobia within their API communities. Participants in Sung and colleagues' (2015) study reported experiences of distress regarding issues of coming out, such as feeling hurt, nervous, fear, and hesitance. Even after coming out, participants experienced stress of continuous denial and invalidation of their sexual identity by others (Nadal \& Corpus, 2013; Sung et al., 2015). API cultural stigma against sexual minority identities influences API families' perceptions of their LGBQ family members. Although there are instances of familial acceptance and open-mindedness (Chung et al., 2006), LGBQ API individuals run the risk of being disowned by their parents when they come out, a consequence that occurred for a queer Korean female participant in Chung et al.'s (2006) study. At times, being open about their sexual identities has resulted in positive outcomes and strengthened parent-child relationships (e.g., closer relationship, honest; 
Chung et al., 2006). However, even among families expressing acceptance and openmindedness, Sung et al. (2015) found LGBQ identities may be required to be contained within the immediate family unit and kept in secrecy from extended families and the broader API community. While isolation or distress may not occur within the immediate family unit, this may continue to result in feelings of isolation from the greater API community. Seventy-two percent of South Asian American respondents reported feeling isolated from their own communities (Choudhury et al., 2005). Thus, it is unsurprising for LGBQ API individuals to not come out to their families. In one study, only half of gay API male respondents reported being open about their gay identity (Wooden, Kawasaki, \& Mayeda, 1983). Those who do not come out to their families, reported feeling like they cannot be their authentic selves or are lying to their family when they are at home, often feeling like they are living double lives (Choudhury et al., 2005; Sung et al., 2015).

Furthermore, acculturation and generational factors can influence LGBQ API individuals' decision-making process about their openness related to their sexual identities. This is apparent in Chung et al.'s (2006) qualitative study of international students, 1.5 generation, and $2^{\text {nd }}$ generation queer Korean women. The primary theme found among the participants was the obligation to be a good daughter. For queer Korean female international students and 1.5 generation immigrants, loyalty to their family was rated as being very important to them; for these participants, being a good daughter meant protecting their parents from "unnecessary pain" by making the personal sacrifice of remaining closeted about their queer identities. However, international students reported the comfort of distance from their parents living in Korea allowed them to be 
good daughters and continue upholding traditional Korean values for their parents, while also being out queer women in the U.S., essentially living double lives. Unlike international students, 1.5 generation participants immigrated with their families as youths; their shared immigration experiences created deep bonds with their family members. Additionally, 1.5 generation participants lived in the same Korean enclaves (e.g., Koreatown) as their parents. The close proximity of their families and Korean communities forced 1.5 generation participants to remain closeted to protect their families, in fear that their families would be ostracized by the Korean community and stripped of their social statuses and social support systems within the community. For these participants, leading double lives may not even be possible. Second generation participants reported higher acculturation due to being born in the U.S., having more exposure to U.S. mainstream culture, and experiencing less connection to the Korean community. Contrasting with previously mentioned participants, most of the $2^{\text {nd }}$ generation participants live away from home and are out to their parents about their queer identity. For queer second generation Korean women, being good daughters meant being open and honest with their parents about their queer identities. They believed conscious openness and honesty, rather than lies and secrecy, will strengthen their parent-daughter relationships. Consistent with individualistic notions of family, $2^{\text {nd }}$ generation participants maintained concepts of independence, choice, self-definition, change, and love. According to Chung et al. (2006), the differences between the groups of participants and their experiences appeared to stem from familial proximity and their acculturation process. Even though this has yet to be studied within trans API populations, it could be that these aspects would develop similarly for trans API people 
because of the similarity in stigmatization of queer/trans identities, API cultural values of filial piety, and generational influences.

While trans API individuals encounter similar challenges as LGBQ API individuals face (i.e., racism within LGBQ communities and homophobia within API communities), these challenges are not exactly the same. Trans API individuals further struggle with racism from the trans community, in addition to transphobia from both the LGBQ and API communities. Transphobia is encountered from both LGBQ and API communities because trans identities transgress not only traditional gender norms but also the preconceived biomedical model of gender and sex. Trans API individuals are not afforded the luxury of having invisible identities like those of minority sexual identities because of the visible social, and often physical, changes that accompany gender transitions. Before discussing trans API experiences, literature on the broader trans community is examined to further contextualize the experiences of this population.

\section{Experiences of the Trans Community in the U.S.}

Under the LGBTQ acronym, research studies often examine trans and LGBQ experiences under the same microscope, yet, frequently fail to yield a representative sample size of trans participants (Blumer, Green, Knowles, \& Williams, 2012; Dargie Blair, Pukall, \& Coyle, 2014; Moradi et al., 2015). For example, Jackson, Johnson, and Roberts (2008) studied the impact of discrimination that limits health care access among LGBTQ elders, and only 2 out of 132 ( $1.5 \%$ of the total sample) participants were trans people. Similarly, in Daley, Soloman, Newman, and Mishna's (2007) qualitative study on the impact of school bullying among LGBTQ youths, only one out of their nine participants was trans-identified. Blumer and colleagues (2012) conducted a content 
analysis of marriage/couple and family therapy-related journals between 1997 and 2009, using "trans" as the search term. Their investigation only yielded 30 articles out of a total of 10,739 articles across all analyzed journals. Even among the 30 articles, only 9 specifically addressed trans issues while the other 21 articles used the LGBT acronym but failed to specifically discuss trans issues. These studies and their respective findings are potentially misleading, perpetuating the conflation of sexual orientation and gender identity, and enabling the erasure of trans experiences (Dargie et al., 2014). To underscore the significance of examining trans and LGBQ experiences, it is important to illustrate, albeit briefly, the differences between trans and LGBQ communities' experiences.

Research studies examining the experiences of cisgender LGBQ people and trans people often conflates the experiences of these two populations, assuming the inclusive and comprehensive sampling of their studies would capture the experiences a presumed monolithic and homogenous LGBTQ community. While some experiences certainly overlap (e.g., transgressing social norms), cisgender LGBQ individuals and trans individuals also have uniquely different experiences (Dargie et al, 2014). Unlike trans individuals, cisgender LGBQ individuals have cisgender privilege, such that trans individuals encounter experiences that cisgender people do not due to their intrinsic cisgender identity (Taylor, 2010). For instance, transitioning for trans individuals often goes beyond "coming out," because of the visible, and often physical, nature of transitioning. Some trans individuals proceed with medically transitioning, which can be a barrier because of the costs associated with the procedures (Chyten-Brennan, 2014; Deutsch, 2014). However, most, if not all, trans individuals engage in social transitioning 
upon coming out, which requires disclosing their trans identity to those around them so their identified gender and pronouns are known and respected (Reynolds \& Goldstein, 2014). Social transitioning may also require trans individuals to perform legal procedures, such as having their names and gender changed on their legal documents including driver's license, social security card, titles, and accounts. Moreover, they may also be required to explain any anomalies in their documentations, such as why one's name on the driver's license is different from their birth certificate (Broadus \& Minter, 2014). To complicate the matter, according to the Transgender Law Center (2016), certain states have laws that forbid trans individuals from legally changing their documentation to match their gender. According to Nadal, Skolnik, and Wong's (2012) qualitative study, systemic microaggressions, such as government-related barriers, were found to be a major theme among trans individuals. Additionally, trans individuals who opt for medical transitioning must jump through "hoops" and overcome systemic barriers to have their transition needs met (Broadus \& Minter, 2014). Unlike cisgender LGBQ people, trans individuals experience the denial of bodily privacy when they are asked intrusive, even interrogative, questions about their genitals, what other sexual organs they may or may not have, and whether they have undergone surgeries (Chung \& Chang, 2015; Nadal et al., 2012). Nadal et al. (2012) also found denial of bodily privacy as a major theme among their trans participants. The denial of bodily privacy and examples of systemic microaggressions, can be observed in recent insurgence of transphobic "bathroom laws" in certain states, which requires individuals to use public bathrooms based on their sex assigned at birth and empowers authorities to inquire about an individuals' genitals to ensure "correct" usage of the assigned bathroom. Trans 
individuals are further different from cis LGBQ individuals due to experiences of transphobia.

Experiences of transphobia. While a complete examination of trans history, conceptualization of trans identities, and deconstruction of gender through various social and cultural theories (e.g., feminist theory, queer theory, gender theory, etc.) is beyond the scope of this literature review, it is important to acknowledge the culturally specific contexts in which trans identity has developed contemporarily. In the United States, trans identities and gender nonconformity have historically been considered pathologies and gender transgressions (Burke, 2011). U.S. society is largely based on Western JudeoChristian culture, which is dualistic in nature in regards to ideologies of sex and gender. Thus, sex and gender are conflated into a single definition and deeply rooted in biblical norms of cisnormativity, such that there are only two sex/gender possibilities-male and female/man and woman — and these binary possibilities are based on observed genitalia (Witten et al., 2003). It is unsurprising that these profoundly engrained Western JudeoChristian ideologies of sex and gender in U.S. history and culture have influenced binary biomedical models of sex and gender (binaochan, 2014). These ideologies influence have consequently impacted the medical, psychological, and social paradigms of trans identities and gender nonconformity (binaohan, 2014; Eckhert, 2016; Erickson-Schroth, Gilbert, \& Smith, 2014).

Trans people defy these ideologies and social norms by simply transgressing from their assigned sex at birth. Historically, the medical and psychological communities have pinned those who transgress society's gender norms and expectations as psychosexually disordered and mentally disturbed (Beemyn, 2013). For example, in 1892, psychiatrist 
Richard von Krafft-Ebing published his famous book, Psychopathia Sexualis, where nonnormative gender expressions were described as inversions. The term transvestism was used to describe men who desires to wear women's clothing (Hamburger, Stürup, \& Dahl-Iversen, 1953). Transsexualism was a listed psychological disorder in the DSM-III and DSM-III-R (Diagnostic and Statistical Manual; American Psychological Association, 1980, 1987). Transsexualism was then changed to Gender Identity Disorder in the DSM-IV and DSM-IV-TR (American Psychological Association, 1994, 2000). During this time, the medical and psychological fields' discourse and research around trans identity continued to be heavily influenced by the binary ideologies of gender. This limited the experiences of trans people into two gender identities and does not allow for much consideration for nonbinary trans people (Budge, Rossman, \& Howard, 2014). Nonbinary trans people and genderqueer individuals make up a significant portion of the trans community. For example, genderqueer people make up between $40-55 \%$ of the trans sample in some studies (e.g., Factor \& Rothblum, 2008; Kuper, Nussbuam, \& Mustanski, 2012). This becomes problematic because it erases whole communities and identities of trans people in discourses. It was only recently with the publication of the DSM 5 that Gender Identity Disorder was changed to Gender Dysphoria and "alternative" gender identities other than binary genders were acknowledged (American Psychological Association, 2013).

Even as medical interventions (i.e., hormones and surgeries) are introduced as a method of assisting individuals transform their bodies into the "opposite sex," trans identities and people continue to be pathologized by a society that perceives them as "freaks," or, at best, sources of entertainment (e.g., Jauk, 2013; Lamble, 2008; Riley, 
Clemson, Sitharthan, \& Diamond, 2012). As described by a participant in Riley et al.'s (2012) semi-structured qualitative study, "as a child I thought transsexual were freaks" (p. 250). Similarly, Jauk's (2013) qualitative study had a participant explain, "They call me a freak, they make me an 'it,' that is dehumanizing" (p. 812). In recent years, the American Psychological Association (APA) and the American Medical Association (AMA) have called for their respective professional fields to be more affirming and validating towards trans identities and people. Similarly, legislation (e.g., trans-inclusive anti-discrimination ordinances) also has been introduced to provide trans people with some protection against various forms of discrimination, such as anti-trans employment and housing policies. However, as Carrera, DePalma, and Lameiras (2012) stated, "[s]imply providing legal rights for trans people does not necessarily provide a way out of the heteronormative straitjacket (p. 1002)." These heteronormative, cisnormative ideologies continue to socialize and pressure trans people to conform to and internalize this society's stereotypical gendered expectations and norms (Dietert \& Dentice, 2013). Trans people's conformity to society's stereotypical gendered expectations and norms also may be related to the desire to be accepted by mainstream, heteronormative, and cisnormative society (Wight, 2011). Furthermore, conformity to expectations and norms may also serve as protection against potential threats, discrimination, and violence (Wight, 2011).

Despite increasing visibility, affirmation, and validation of trans people in health care fields and mainstream society, anti-trans discrimination and violence are still rampant. Trans individuals experience high rates of discrimination (Stotzer, 2009), and report more instances of discrimination than cisgender LGBQ individuals ( $\mathrm{Su}$ et al., 
2016). Grant et al. (2011) conducted the largest trans discrimination survey to-date and found that discrimination was an ubiquitous experience among their trans participants' daily lives (e.g., prevalent in all aspects of daily life, such as in schools, places of employment, housing, public spaces, and by the police). Trans participants in Jauk's (2013) ethnographic qualitative study described experiences of discrimination as a "Catch 22 ," such the consequences associated with living as their authentic selves, including institutional discrimination and ever-present possibility of violence, can lead to negative mental well-being. Trans individuals reported being at higher risk of violence and assault when they "look trans," and that trans individuals not only feel unsafe in public but also within their own homes (Jauk, 2013, p. 815). However, while conforming to mainstream, heteronormative, cisnormative expectations may provide safety from transphobic discrimination, psychological distresses and suicidal ideation are often the results of being able to live authentically (Jauk, 2013).

Much like other forms of discrimination, anti-trans discrimination is experienced at both personal and systemic levels. Nadal and colleagues (2012) determined twelve themes of personal and systemic anti-trans microaggressions that were experienced by trans people (see Table 1). Among families, Nadal et al. (2012) found that many of the microaggressions pertained to participant's physical transition process, names and pronouns, and how they expressed their gender; these microaggressions were often coupled with rejection and even hostility. This is consistent with Grant et al.'s (2011) findings where fifty-seven percent of the 6,456 trans participants reported experiencing rejection from their family and nineteen percent reported physical domestic violence perpetuated by a family member. Similarly, verbal victimization perpetuated by family 
and parents can occur in childhood (e.g., being called "sissy" or "tomboy"), where female trans youths report higher rates of physical victimization by family than male trans youths (Grossman, D’Augelli, Howell, \& Hubbard, 2005; Grossman, D’Augelli, Salter, \& Hubbard, 2005). Bariola et al. (2015) found that a lack of family support was associated with psychological distress. Victimization and discrimination also are associated with high levels of psychological distress (e.g., Bariola et al, 2015; ClementsNolle, Marx, \& Katz, 2006; Maguen \& Shipherd, 2010; Reisner et al., 2016).

\section{Table 1}

Interpersonal and systemic anti-trans microaggression themes

\section{Themes}

1. Use of transphobic and/or incorrectly gendered terminology

2. Assumption of universal transgender experience

3. Exoticization

4. Discomfort/disapproval of transgender experience

5. Endorsement of gender-normative and binary culture or behaviors

6. Denial of existence of transphobia

7. Assumption of sexual pathology or abnormality

8. Physical threat or harassment

9. Denial of individual transphobia

10. Denial of bodily privacy

11. Familial microaggressions

12. Systemic and environmental microaggressions

Experiences of discrimination also have been associated with symptoms of posttraumatic stress disorder (PTSD), even after controlling for prior exposure to traumatic events (Reisner et al., 2016). Trans individuals are more likely to report psychological distress (e.g., depression, suicide) compared to cisgender LGBQ individuals ( $\mathrm{Su}$ et al., 2016). Thus, it is not surprising that forty-one percent of trans participants have reported attempting suicide at least once in their lifetime (Grant et al., 2011). However, these findings describe general experiences of the overall trans community and/or where the 
majority of the sample is White. They do not, necessarily, fully describe the experiences of trans people of color, because unlike White trans people, trans people of color also experience the intersection of their race/ethnicity with their trans identity.

Experiences of racism. In the field of counseling and counseling psychology, empirical literature has had a pattern of oversampling White participants while underutilizing participants of color in their samples (Delgado-Romero, Galvan, Maschino, \& Rowland, 2005). Singh and Shelton (2011) found the empirical counseling literature related to LGBTQ communities mirrors the findings of Delgado-Romero and colleagues, with only two of the twelve studies included in the content analysis having a specific focus on people of color's experiences. Given that the vast majority of the trans literature continues to be dominated by White trans individuals' narratives, it is important to consider the narratives of trans people of color.

According to the National Transgender Discrimination Survey, trans people of color reported higher rates of discrimination, violence, psychological distress, and rejection in most aspects of daily life (e.g., health care access, employment, education, etc.) compared to their White counterparts (Grant et al., 2011). Correspondingly, Erich, Tittsworth, Meier, \& Lerman (2010) found twelve domains in which trans people of color experience gender-related discrimination: (a) social services; (b) employment; (c) health care; (d) mental health care; (e) housing; (f) immigration; (g) interactions with police officers; (h) educational environments; (i) public transportation; (j) identity documents; (k) child custody; and (l) family interpersonal relationships. Among ninety trans participants in Lombardi's (2009) study, race was found to be associated with transphobic discriminatory events, such that Black trans participants had higher rates of 
reporting transphobic events compared to White trans participants, who had the lowest rates of reporting transphobic occurrences.

The intersection of race/racism and trans identities/transphobia in experiences of discrimination, and as such the differences in experiences between White trans people and trans people of color, is apparent. U.S. cultural values of what is deemed gender transgression and gender congruent amongst people of color is deeply embedded into our society and culture, and influences the intersection of transphobia and racism. Consider the experiences of Black individuals and trans individuals as related to police harassment and brutality, which have deep impacts among both communities. Police harassment and brutality are more likely to occur among people of color, particular among Black individuals (Chaney \& Robertson, 2013). Thus, while $22 \%$ of trans respondents reported experiences of police harassment, only $18 \%$ of the respondents were White while the rate was much higher among trans people of color with the highest rates being among Black and multiracial trans people (38\% and 36\%, respectively; Grant et al., 2011). While the literature on police brutality, racism, and hetero- and cisnormativity is beyond the scope of this dissertation, one could argue the higher rate of police brutality among trans people of color is influenced by not only the racism involved, but also the gender transgression within an already targeted black or brown body. For instance, the intersection of the criminalization of black bodies and the additional perception of transwomen as sex workers can lead to the increased likelihood that Black transwomen are subjected to police harassment and wrongful arrests (often due to the perceptions of them being sex workers; Graham, 2014; Marksamer, 2008). These experiences of transphobia and racism among trans people of color is consistent with the minority stress literature, which 
indicates the more minority identities an individual has, the more stress the individual experiences due to their minorities identities (e.g., Bowleg et al., 2003; Hayes et al., 2011; Meyers, 2010). Due to a White ethnocentric trans literature, the in-depth examination of the intersectionality of transphobia and racism is often overlooked.

Protective factors. While discriminatory instances of transphobia and racism are inherent to the lives of trans people and trans people of color, there are protective factors that help buffer the psychological impact of such adversities. Budge et al. (2014) found genderqueer and nonbinary trans individuals who reported having more social support also used more facilitative coping strategies (e.g., active help seeking, positive reframing) and reported less depression and anxiety. Budge, Orovecz, and Thai (2015) found positive interpersonal reactionary responses (e.g., from family members) contributed to transmen's positive emotional experiences during their trans identity developmental process. Affirming, supportive family relationships have been found to be related to higher levels of life satisfaction, as well as self-esteem and well-being among trans individuals (Erich, Tittsworth, Dykes, \& Cabuses, 2008). When asked to reflect on their experiences and needs as gender transgressing children, adult trans participants reported a number of themes (Riley, Clemson, Sitharthan, \& Diamond, 2013). One of the most common childhood needs described by Riley et al.’s (2013) adult trans participants was the need of unconditional parental love and an open, sensitive environment to speak to their parents about their feelings. Another need that surfaced in the study was the need for transcending of cultural heritage and religious values by their parents, because familial culture influences and religious persecution were barriers that prevented parental acceptance of childhood gender transgression (Riley et al., 2013). 
To complicate the narrative, the intersectionality of race and trans identities sheds light on the differences in cultural factors that may influence the interpersonal relationships between trans people of color and their family. White trans people are more out to their families about their trans identity than most trans people of color (Grant et al., 2011). For example, based on a study of almost 6,500 trans individuals, $57 \%$ of White trans individuals are out to their families compared to $47 \%$ of trans API individuals (Grant et al., 2011). White trans people experience less family rejection than trans people of color (e.g., $39 \%$ of White trans individuals as opposed to $49 \%$ of multiracial trans individuals; Grant et al., 2011). Although family members, relationships, and interactions can contribute to the overall traumatic experience of discrimination for trans people of color, these individuals and interactions can also engender resilience in the face of such trauma. Among eleven trans participants of color, Singh and McKleroy (2011) qualitatively found familial relationships of acceptance to be a central pillar of the participants' resilience to experiences of trauma and discrimination. Furthermore, successfully navigating these relationships from initial rejection to eventual acceptance helped participants feel protected and heal from their traumatic experiences (Singh \& McKleroy, 2011).

Positive familial relationships also influence the evolving racial and trans identity development, as was the case among thirteen trans youths of color in Singh's (2013) phenomenological study. Development of racial/ethnic and trans identities also has been found to be essential in trans people of color's resilience (Singh, 2013; Singh \& McKleroy, 2011). Singh and McKleroy (2011) identified "Pride in One's Gender and Ethnic/Racial Identity" as a common theme shared by trans participants of color as 
salient in their resilience against traumatic discriminatory events. One participant described the development of her sense of pride:

Society put me in a place where I am a minority and doesn't think I have the talent or potential because of who I am [racially/ethnically]. When I was a child, didn't think my ethnicity was anything to be proud of - I was looked down on because I was Black. And then being transgender made it even worse. Now that I am older, I am proud of everything I am. I am an African American transsexual woman. NO one can tell me I am less than anyone else. That helps me no matter what bad things happen to me.

Some days, just getting out of bed is a revolutionary act to deal with the world. And I make sure I get out of bed (p. 38).

Simultaneous racial and trans identity developmental processes, such as

awareness and self-definition, were also found to be a critical component of the resilience of trans youths of color (Singh, 2013). Twelve out of the thirteen trans youth participants of color described self-definition as a source of empowerment that lead to developing pride in themselves and their identities. This sense of empowerment of their identities also allowed them to feel connected with both their racial and trans identities, as well as feel confident in their expressions of their identities. There is evidence of the importance of racial and trans identity developmental processes and the negotiation of these identities in trans people of color. However, these studies only examine the outcomes of such identity developmental processes and negotiations with oppression, rather than how their trans participants of color navigate and negotiate their racial and trans identities during their identity developmental processes and spaces. Furthermore, these studies only have a few API trans participants in their samples or lacked them altogether. Reviewing the few available trans API research that is based on U.S. populations is important in order to understand the experiences of trans API individuals' encounter with U.S. trans culture and their culture of origin to better contextualize their identity processes. 


\section{Experiences of Trans API People}

Trans API literature related to identity navigation and negotiation is dominated by the narratives of trans API individuals living outside of the U.S. (e.g., Kalra, 2012; Vanderlann et al., 2015; Winter, 2006; Winter et al., 2007). For example, both Poasa (1992) and Roen (2001) found that their fa'afafine participants rooted their understanding and negotiation of their fa'afafine in their Samoan culture. Roen described one of the Samoan participant's self-conceptualization of having a fa'afafine identity, “cultural identity precedes gender/sexuality identity in political importance, but the two are intrinsically linked: one does not make sense without the other" (p. 257). Similarly, Sinnott's (2004) ethnographic book illuminated the difference in cultural conceptualization of gender and sexuality among Thailand's toms and dees (Thai female gender and/or sexual identities with specific gender roles/expectations associated to each identity; for full explanation of tom and dee identities, see Sinnot's Toms and Dees: Transgender Identity and Female Same-Sex Relationships in Thailand), such that these individuals could only understand their tom/dee identities in relation to their own culture. Simultaneously, these individuals also described difficulties in understanding and relating to Western notions of gender, sexuality, and gender roles/expressions. For example, Sinnott described an exchange with a Thai tom about gender and sexual identities in the U.S.:

She turned to me ask asked what farang (Westerners) are like: 'Do they have toms and dees too?' I said that as far as I knew, there were not such strong gender identities anymore, not like tom and dee in Thailand. Kot and her friends looked at me blankly at first, then glanced at each other, seemingly at a loss for words, and started giggling. 'How do you know what to do then?' one chortled (p. 77).

While these narratives are important and crucial to the overall trans literature, the 
narratives of trans API individuals in the U.S. continue to be overlooked. Furthermore, the literature specifically related to trans API individuals in the U.S. is limited to focusing mostly on the experiences of discrimination rather than identity related development.

Like other trans people of color, trans API individuals encounter racial and transrelated discriminations, unfortunately, their experiences are often aggregated with the experiences of other trans people of color and presented as homogenous experiences in empirical research (e.g., Bith-Melander et al., 2010; Nemoto, Operario, Keatley, Han, \& Soma, 2004; Singh, 2013; Singh \& McKleroy, 2011; Xavier, Bobbin, Singer, \& Budd, 2005). Experiences of trans API individuals are also often combined with the experiences of LGBQ API populations, even though these studies examine experiences related to sexual orientation/identity rather than gender identities (e.g., Choudhury et al., 2009; Ohnishi, Ibrahim, \& Grzegorek, 2006; Szymanski \& Sung, 2010; Wong, Chng, Ross, \& Mayer, 1998). It is challenging to find trans API experiences in the U.S. that are not embedded in LGBQ literature. When separated and examined individually a clearer picture of the experiences of trans API individuals can be viewed.

While trans API individuals only made up a small percentage $(0.03 \%)$ of the largest national trans discrimination survey to date, the descriptive statistics from the study are still eye-opening and contributes awareness and information to the current trans API body of research (Grant et al., 2011; National Queer Asian Pacific Islander Alliance, NQAPIA, 2011). According to the survey, trans API participants live in extreme poverty (household income of under $\$ 10,000 /$ year) and have the highest rate of poverty among all trans participants of other races living in the U.S., six times higher than the general API population, and over four times higher than the general population (Grant et al., 2011; 
NQAPIA, 2011). Additionally, even though $44 \%$ of trans API participants reported acceptance from their family units, $56 \%$ reported having attempted suicide at least once in their lifetime due to discrimination compared to $41 \%$ of the overall trans sample and 1.6\% of the general population (Grant et al., 2011; NQAPIA, 2011). The discrimination they experience can manifest among the White LGBTQ communities and API communities, as well as other LGBTQ communities of color. Sixty-seven percent of trans API participants in Dang and Hu's (2005) study reported being discriminated against based on their gender identity in general, but all trans API participants reported discrimination based on their gender expression (compared to $43 \%$ of cisgender API gay men and $58 \%$ of cisgender API lesbian women) and race (compared to $85 \%$ of cisgender API gay men and $78 \%$ of cisgender API lesbian women). Trans API participants were also more likely to experience discrimination within the White LGBTQ community and LGBTQ communities of color than their cisgender API gay and lesbian counterparts (Dang \& Hu, 2005). Interestingly, Dang and $\mathrm{Hu}$ (2005) also found that trans API participants did not differ from cisgender gay and lesbian participants in experiences of discrimination from their API communities. This may be due to both trans and cisgender API individuals encountering API communities that are influenced by similar API cultural values such as values related to gender and sexuality. U.S. trans identities may conflict with API cultural values due to the collectivistic nature of those values and the individualist nature of U.S. trans culture.

With the U.S. being a fundamentally individualistic society (Triandis, 2001), it is not surprising that the conceptualization of trans identity and the process by which trans identity develops in the U.S. is largely influenced by individualism. U.S. culture places 
heavy emphasis on self-determination, individuality, and independence (Triandis, 2001). The influence of individualism can be found in the culture of the trans community in the U.S. and the scholarly trans literature.

A common theme found in the trans community, trans research, and the literature on counseling trans populations is the strive for self-determination - to be able to define oneself and one's identity independent of the social norm and messages one encounters (e.g., Diamond, Pardo, \& Butterworth, 2011; Israel, 2004, 2005; Mathy, 2001; McPhail, 2004; Monro, 2000). For example, Israel (2004) stressed the importance for health care providers in advocating for trans people's right to self-determination of their gender and trans identities, and as a means of depathologizing their identities. Collazo, Austin, and Craig (2013) suggested clinicians facilitate and strengthen trans clients' sense of selfdetermination, where their self-determination gives them the power and autonomy over the self-conceptualization of their gender identities and the timing/context of the disclosures of their identities to others. Guidelines from various health care fields (e.g., American Counseling Association, American Psychology Association, World Professional Association of Transgender Health) recommended providers afford trans individuals with autonomy and self-determination about their identities and bodies, while being sensitive to their possible gate-keeping positions (American Counseling Association, 2010; American Psychological Association, 2015; Singh \& Burnes, 2010). Self-determination was considered a component of multicultural competence and social justice, particularly among the field of counseling psychology; lack of self-determination would only further maintain society's oppressive status quo (Vera \& Speight, 2003). Individuals in the trans community are increasingly vocal about their rights to self- 
determination of their own gender identities (Bockting, Robinson, Benner, \& Scheltema, 2004). Belief in their self-determination and autonomy has resulted in trans individuals being outspoken against the medical and mental health systems' gate-keeping of their identity and their access to gender confirmation interventions (Bess \& Stabb, 2009; Fontaine, 2002). However, independence and autonomy are rooted in individualism. Interdependence and autonomy assume and celebrate trans individuals making decisions for themselves and their bodies, becoming more self-reliant, and asserting themselves to get needs met (e.g., asking for respect regarding gender identity, pronouns, transitional processes, etc.). Such independence and individualism, from which selfdetermination stems, vastly contrasts the interdependence that derives from collectivistic API cultural and familial values, and may not be a viable option for trans API individuals. For example, considering API cultural and familial values related to filial piety, assertively requesting the use of correct pronouns or correcting misgendering languages from parents may not always be a possibility among trans API individuals because that would require confronting parents and/or elders. The direct challenging of parents, elders, and community members by younger individuals (regardless of age) is frowned upon, defies filial piety, and potentially damages the family's face (e.g., being perceived as not being able to control one's own children/family member, lacking selfcontrol, having no moral values, etc.). Trans API individuals' experiences may further be complicated by the feeling that it is their family duty to make personal sacrifices by enduring transphobic transgressions from their families, due to their collectivistic cultural and family values. These individuals may internally struggle with having to choose between their own self-determination and their API family and cultural community, in 
addition to encountering external discriminatory environments from their families and communities.

\section{Literature Methodology Overview and Limitations}

Currently, the majority of the emerging trans literature consists of conceptual and theoretical papers, with 54\% of 960 published trans-related articles between $2002-2012$ are nonempirical (Moradi et al., 2016). While the empirical literature is beginning to grow, the methodologies in the literature frequently used are a variety of qualitative approaches and/or elementary quantitative statistical analyses. These methodologies vary depending on the variables or topic(s) under examination. For example, literature on trans identity development or trans resilience regularly utilize qualitative approaches (e.g., grounded theory, phenomenological, interpretative, focus groups, case studies, etc.) to describe the process in which trans people develop their gender identities or resilience (e.g., Dietert \& Denice, 2013; Grossman, D’Augelli, \& Frank, 2011; Moody et al., 2015; Pollock \& Eyre, 2012; Singh, Hays, \& Watson, 2011; Singh, 2013; Singh \& McKleroy, 2011). Qualitative approaches have been used to describe experiences within different environments, such as in places of employment (e.g., Schilt, 2006) and in the home (e.g., Norwood, 2012; White \& Ettner, 2004). Similarly, quantitative studies have also examined many of these processes.

Quantitative trans literature spans a multitude of topics, such as experiences with transphobia (e.g., Erich et al., 2010; Grant et al., 2011; Lombardi, 2009), discrimination related post-traumatic stress disorder symptoms (e.g., Reisner et al., 2016), suicidality (e.g., Clements-Nolle et al., 2006), and mental health disparities (e.g., Su et al., 2016). The majority of the existing quantitative trans literature primarily utilizes straightforward 
statistical analyses, such as descriptive stats, t-tests, chi-square tests, correlation, ANOVA, and/or multiple regression (e.g., Bariola et al., 2015; Clements-Nolle et al., 2006; Erich et al., 2010; Lombardi, 2009; Su et al., 2016). A few studies have used more advanced statistical analysis, such as structural equation modeling (e.g., Barr, Budge, \& Adelson, 2016; Budge, Adelson, \& Howard, 2013). Additionally, many of these studies employ non-psychometric (often dichotomous) questions and/or psychometric instruments (originally normed on cisgender populations) that were adapted for trans population (e.g., Erich et al., 2010; Maguen \& Shipherd, 2010; Reisner et al., 2016; Su et al., 2016). Together, these quantitative and qualitative studies further contribute to the knowledge and understanding of trans experiences. However, both the qualitative and quantitative methodologies utilized in the current literature have major limitations in the study of trans API experiences.

While these qualitative studies are integral for the understanding of trans experiences, unfortunately, these studies provided insufficient information on the experiences of trans API individuals due to their underrepresentation or complete absence from the samples. Even among qualitative studies regarding trans people of color, there continues to be a dearth of API voices and their experiences. Singh and McKleroy's (2011) qualitative study, which examined the experiences of resilience among trans people of color, had a sample size of eleven participants, none ( $0 \%)$ of which were API. Similarly, out of a sample of thirteen participants, Singh's (2013) study of trans youths of color yielded only two (15.4\%) API participants. Some trans studies do not report race and/or ethnicity at all (e.g., Dietert \& Denice, 2013; Poteat et al., 2013; White \& Ettner, 2014). In addition to the limitations related to race and/or ethnicity and the lack of trans 
API sampling, many of these studies further fail to sample nonbinary trans identities (e.g., Grossman et al., 2011; Nadal et al., 2012; Poteat et al., 2013; Singh \& McKleroy, 2011). These limitations are also common occurrences in the quantitative methodologies used in the literature.

Just as with the qualitative literature, quantitative literature also struggles methodologically to include trans API experiences. This makes such findings limited in their generalizability for trans API individuals. For instance, out of nearly 6,500 trans participants, the National Transgender Discrimination Survey, the largest study on the experiences of discrimination among trans people, was only able to collect a sample size of $213(0.033 \%)$ trans API participants (Grant et al., 2011). Similarly, Bockting, Miner, Swinburne Romine, Hamilton, and Coleman's (2013) study on stigma, mental health, and resilience among trans people consisted of a sample size of 1,093 participants, but only $17(1.6 \%)$ of the sample were trans API individuals. Compared to $6 \%$ of the total U.S. population represented by API individuals (Hoeffel et al., 2012), these studies do not have representative samples of API participants. Some studies have such small sample sizes of people of color in general that they have aggregated their participants of color into one all-encompassing "non-white" or "ethnic minority" category, with no explanation or insight of the race and/or ethnicity of the participants that have been placed into it (e.g., Clements-Nolle et al., 2006; Maguen \& Shipherd, 2010; Su et al., 2016). Other studies have no trans API participants in their sample collection (e.g., Lombardi, 2009; Reisner et al., 2016). Additionally, like the limitations of many qualitative trans studies, many quantitative studies only pull from binary-identifying trans samples, meaning only sampling from male-identifying or female-identifying trans 
individuals (e.g., Bariola et al., 2015; Bockting et al., 2013; Erich et al., 2008; Testa et al., 2012). The lack of information on nonbinary trans individuals not only fails to shine light on the totality of the trans community, it further does a disservice to the trans community by perpetuating gender binary-centrism, essentially eliminating the experiences and voices of nonbinary trans people in the trans literature. Thus, it is critical to fill in the gaps in research by focusing on trans API experiences to help in moving the research forward. This inclusivity and focus can lead to a more holistic understanding of the trans community, it can also lead to the development and culturallysensitive, responsive provision of comprehensive, inclusive trans health care services and policies.

\section{Summary}

API cultural values of collectivism, filial piety, saving face, and personal sacrifice set the social norms and expectations for the API communities, families, and individuals. While acculturation and differences in generations may impact the behavioral acculturation of API individuals, acculturation related to cultural values are much more stagnant and slower to change over time (Bacallao \& Smokowski, 2009; Kim \& Omizo, 2006; Marín \& Gamba, 2003; Ryder et al., 2000; Szapocznik et al., 1978). These gaps in acculturation and values not only can cause bicultural stress for the individual, they can also further cause familial conflict among API individuals (e.g, Pyke, 2005; Xia et al., 2013). These factors impact how API individuals experience and process their LGBQ identities. However, unlike LGBQ identities, trans identities may not be invisible identities and cannot hide their gender identity from their families and communities. Despite trans individuals becoming increasingly more visible, publicly out, and 
outspoken about their identities and needs in recent years, trans communities continue to experience extremely high rates of discrimination and psychological distress. The trans research looks at forms of discrimination (i.e., racism and transphobia) as a whole, but it does not necessarily look at how racism and transphobia influence and intersect with one another. Current research also has not examined fully how racism and transphobia influence the cultural contexts in which trans people of color, particularly trans API individuals, experience their identities. The influence of individualism can be observed in trans API individuals' self-determination, autonomy, outspokenness, and individuality. However, these values conflict with dominant API cultural values. For trans API individuals in the U.S., whose identities are both trans and API, there may be an internal struggle with these opposing value systems and this internal struggle may impact their psychological well-being. In addition, they also experience external struggles occurring within the family and the trans community. The current trans literature in psychology focuses on the systemic experiences of trans API individuals without or with limited cultural and familial lenses. Furthermore, the employed methodologies result in shortcomings in the descriptions of trans API individuals' experiences.

\section{Present Study}

To date, this investigator has not found empirical U.S. studies examining the process to which trans API individuals navigate and negotiate these seemingly conflicting identities, especially considering their acculturation and generational factors. While it is possible to make inferences about their experiences using the existing literature, they may not accurately capture the comprehensive experience of navigating and negotiating cultural values and identities among trans API individuals. Additionally, the influence of 
acculturation and generational factors must be taken into consideration. Therefore, this study is to gain a fuller understanding of the experiences of trans API individuals, which can contribute to the emerging theories and additional research. Thus, in order to begin developing this knowledge of trans API individuals lived experiences a phenomenological qualitative methodology is proposed. Consistent with the phenomenological approach, hypotheses are not employed. Instead, this phenomenological qualitative study seeks to answer the following research/guiding questions:

1. As trans API individuals develop their identities, what does the process of negotiating their trans and API identities involve?

2. As a trans API person, what does the process of navigating trans spaces involve? API spaces?

3. What aspects related to API experiences (e.g., cultural values, acculturation, generational factors, etc.) influence trans API individuals' identity processes and their experiences? 


\section{CHAPTER III}

\section{METHODOLOGY}

This chapter details the methodology used to examine the experiences of trans and API people, with the focus on their identities and the spaces of family and the trans community. Additional cultural factors (e.g., cultural values, acculturation, and generational factors) as related to trans API individuals was examined. This chapter describes the following: (a) the participants, (b) the recruitment process for this study, (c) the protocol developed for use, (d) the procedure used to conduct the qualitative interviews, and (e) the method of phenomenological analysis.

\section{Sampling}

Because the study sought to better understand the cultural identity negotiation and navigation of trans API individuals, participants with these identities are necessary. The inclusion criteria for this current study were:

1. Participants must be 18 years of age or older;

2. Participants must live in the U.S.;

3. Participants must identify as trans (including any gender diverse identities that fall under the nomenclature of trans);

4. Participants must identify as Asian, Asian American, and/or Pacific Islander (e.g., East/Southeast/South Asian and Pacific Islander/Polynesian ethnicities);

5. Participants must have grown up/raised full-time in an API household. The trans community is a largely hidden population, particularly related to 
accessibility for research (Rosser, Oakes, Bockting, \& Miner, 2007). Because trans API individuals make up a small percentage of the trans community this population remains further unseen. Thus, online recruitment of trans API participants was employed; the use of online sampling is not only commonplace in social science methodologies it is also advantageous for the recruitment of trans participants as it allows for more accessibility (Rosser et al., 2007).

Initial round of recruitment was conducted by sending recruitment flyers and letters to online local, regional, and national trans and/or queer API organizations and communities (e.g., trans-related social media groups). Later rounds of recruitment were conducted by sending flyers and letters through organization and community listserv emails. To take advantage of the vast diaspora of the API community and to ensure inclusion of diverse experiences among trans API individuals, recruitment was not limited to any particular geographic location in the U.S. Recruitment flyers contained brief information about the study, the researcher, the study's process, inclusion criteria, and a link to the study's sign-up webpage. Additionally, information about the researcher's gender and API identities was included to encourage participation and address any concerns.

\section{Participants}

A sample of ten participants was collected. In general terms, five participants identified as male/man/transmale/transman, four participants identified as gender nonbinary (e.g., genderqueer, genderfluid, etc.), and one participant identified as female/woman/transfemale/transwoman. Participants also were able to share their specific gender identities (see Table 2). Participants' ages ranged from 21-42 years 


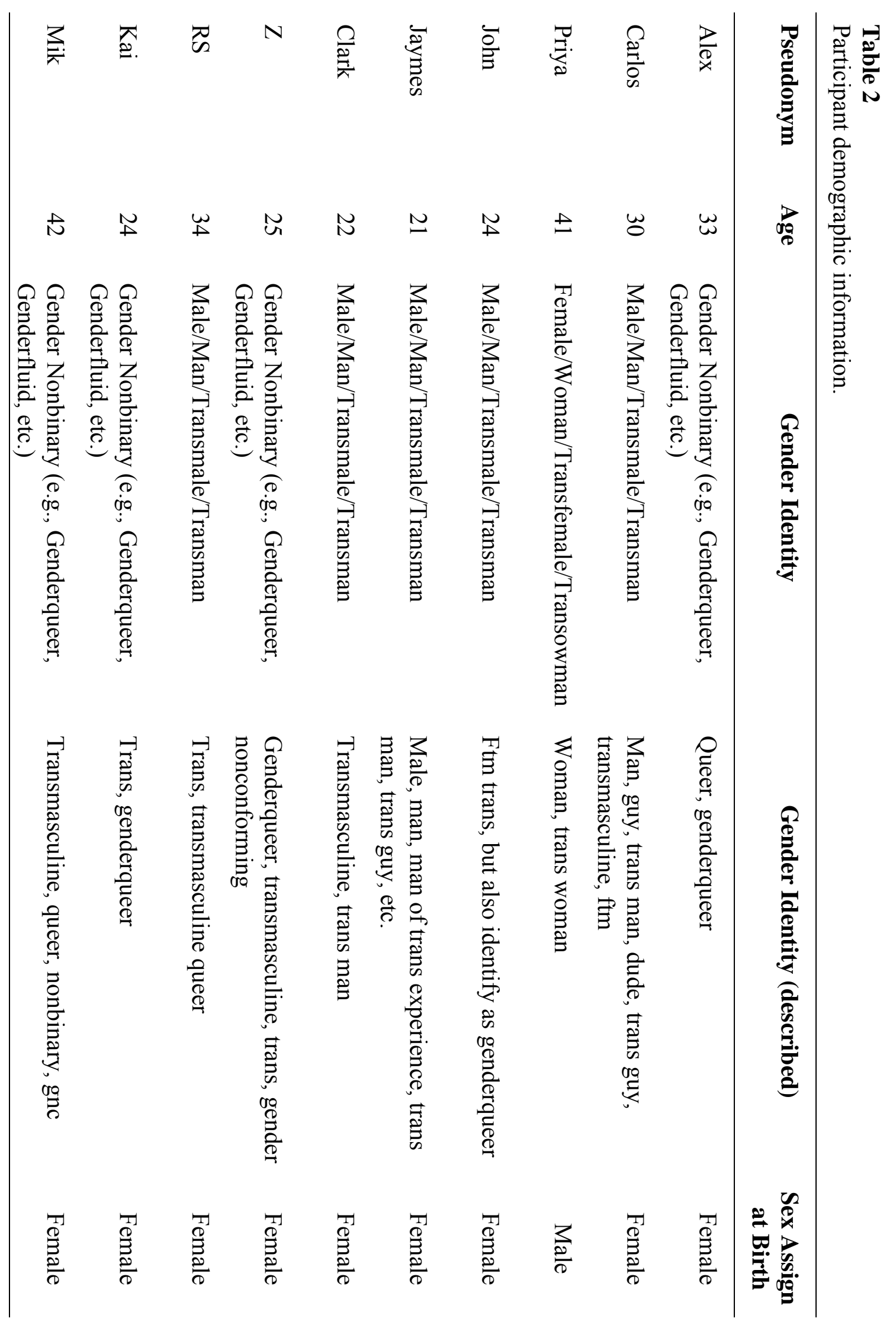




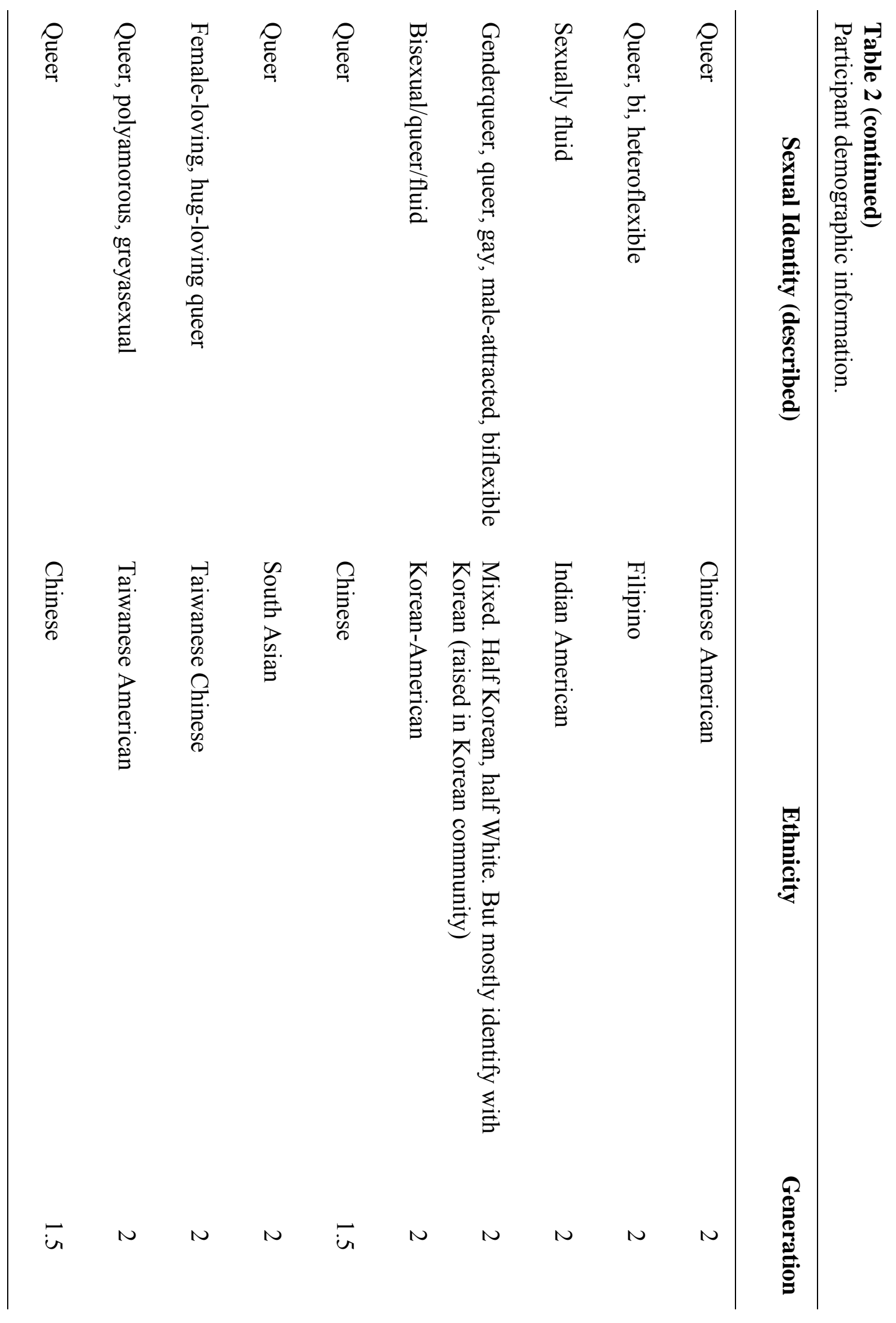


( $M=29.6 ; S D=7.68)$. Eight participants were second generation, while two participants were 1.5 generation.

\section{Research Protocol}

In addition to the demographic and descriptive questions (Appendix A), the semistructured interviews used a protocol which contained broad open-ended questions pertaining to the research questions (Appendix B). In accordance with phenomenological qualitative methodology, there were two broad generation questions that asked participants: "what" their experiences are regarding the phenomenon, and "how" they experienced the phenomenon relating to the contexts that affects and influences their experiences (Creswell, 2013; Moustakas, 1994). Therefore, the protocol contained questions that addressed the "what" and "how" of their lived experiences related to their navigation and negotiation of their trans and API identities and spaces. This included their experiences with their communities and families, as well as the cultural values and identities related to these respective contexts. The protocol also specifically addressed participants' experiences with acculturation based on their generation, and how their acculturation influenced their values, identity development, and identity negotiation. Lastly, the protocol further asked about their psychological and emotional experiences during their identity development and negotiation process. The semi-structured interview process allowed for the interviewer to modify the protocol for further probing of the phenomenon as needed and occurred throughout the process.

\section{Procedure}

The study's webpage had a link to the informed consent form, demographic questions, and sign-up link. The sign-up link allowed participants to leave their contact 
information in order for the researcher to contact them and schedule either an in-person or Skype face-to-face interview for the study. Completion of the informed consent form, demographic and descriptive questions, and leaving contact information was the indication of the individuals' interest and agreement of participation in the study. Upon reviewing the demographic questions to ensure inclusion criteria have been met, the researcher contacted the participants via email to set up an interview time. Due to a nation-wide recruitment, none of the participants were able to attend in-person interviews. All face-to-face interviews were conducted over Skype. All participants were asked the same main questions of the protocol (Appendix B), with additional probing questions asked, as needed and as appropriate, for each participant's interview. The semi-structured interviews lasted between 60 to 120 minutes. All interviews were audio-recorded using an audio recorder. All interviews were transcribed verbatim by the researcher and team of transcribers, which were used in the data analysis.

\section{Analytic Method}

Phenomenological qualitative methodology, specifically Moustakas's (1994) transcendental phenomenological approach, was used to analyze data for this study. Moustakas's (1994) transcendental phenomenology was chosen because of the empirical nature of the approach and its focus on the essence of the phenomenon/lived experiences of the participants. Data analysis was conducted by the sole researcher. Using the transcribed participant interviews, the researcher examined each line of the transcript to identify significant statements related to how the participant experiences the phenomenon. For each interview a list of statements was collected; this process is also known as horizonalization, or horizonalization of the data (Moustakas, 1994). This 
process also allows readers to easily observe the wide range of experiences and perspectives relating to the phenomenon. These significant statements were then clustered/coded into themes, or "meaning units," which are larger units or higher levels of information (Creswell, 2013; Moustakas, 1994). Next, these collected themes were synthesized into composite textural descriptions of the phenomenon experienced by the participants (i.e., what happened) and composite structural descriptions of the phenomenon through contextual lenses (i.e., how did the experience happen). Finally, both textural and structural descriptions were incorporated together to construct composite descriptions of the phenomenon, which is the "essence" of the experiences.

The thematic portrayals of the phenomenon were discussed in the results chapter. Each theme was presented and defined, with details of the themes and descriptions the horizontalization data that are clustered under each theme. To summarize the thematic findings, the discussion chapter included the composite textual description, the composite structural description, and the textural-structural synthesis. The composite textural description illustrated the identity process of navigation and negotiation for trans API participants. The composite structural description elucidated how trans API participants experienced the process. Lastly, the textural-structural synthesis integrated the composite descriptions into a comprehensive narrative of the phenomenon. The narrative described the essence of the phenomenon of navigating and negotiating trans API identities.

\section{Trustworthiness}

Lincoln and Guba (1985) posits that the trustworthiness in qualitative research studies is established by meeting four major criteria: credibility, transferability, dependability, and confirmability. The authors suggest various techniques that can be 
used to achieve these criteria. The following describes the techniques that were implemented in the current study to meet criteria and establish trustworthiness.

Credibility. According the Lincoln and Guba (1985), credibility is the equivalent of internal validity. At its essence, credibility strives to ensure the accuracy of the data in accordance to the phenomenon under examination. To achieve credibility, the research utilized member checking.

Member checks. The interview transcripts were sent to its respective participant for member checking, evaluation, and validation. This gave participants the opportunity to make any modifications and/or clarification to ensure accuracy of the information. Guba (1981) describes member checks as "the single most important action inquirers can take, for it goes to the heart of the credibility criterion" (p. 85). Participants' feedback were incorporated into the data. Participants were also given the opportunity to choose their pseudonym to be used in the study. While not all participants partook in this opportunity, this allowed for the data to not only be as accurate to the participants' lived experiences but also to how participants wanted to be presented.

Transferability. While qualitative methods do not consider it possible to arrive at a universal "truth," they recognize that phenomena are contextually-bound (Guba, 1981; Lincoln \& Guba, 1985). In order to determine transferability, descriptions or interpretations of the context must be given in detail.

Thick description. Having thick descriptions of the context will allow for comparison of various contexts to be possible, permitting the contemplation of transferability and applicability. By the nature of transcendental phenomenology, thick descriptions were provided in the structural, textual, and synthesized descriptions, as well 
as throughout the thematic descriptions.

Dependability. This criterion concerns the stability of the data, while also allowing for instabilities to arise due to multiple lived realities (Guba, 1981). To ensure stability, the study's methodological and analytical processes was thoroughly detailed and scrutinized. Shenton (2004) describes having such detailed attention and reporting of the research design and process "enables readers of the research report to develop a thorough understanding of the methods and their effectiveness" (p. 71). This also allows readers to follow along with the researcher's running account of the chronological research process of data collection and analysis (Creswell \& Miller, 2000). It will further provide insight into the researcher's decision-making process, allowing for future replicability (Guba, 1981; Lincoln \& Guba, 1985; Shenton, 2004).

Audit trail. The researcher created an audit trail of the researcher's experiences of the interview process and the decisions that were made along the way. This was accomplished utilizing field notes after each interview, as well as during the auditing process. Readers can examine the process of the collection the data, including emotions and thoughts that may have manifested throughout the interviews.

Confirmability. Confirmability refers to the objectivity of the researcher, where the researcher's biases and assumptions do not intrude into the data. It further ensures that the resulting phenomenon are of the participants' lived experiences (Guba, 1981; Lincoln \& Guba, 1985; Shenton, 2004). Two techniques were used to meet this criterion: confirmability audits and bracketing/reflexivity.

Confirmability audit. Correspondingly to the dependability audit, a confirmability audit seeks to determine the confirmability of the data. Guba (1981) 
described this process as "certifying that data exist in support of every interpretation and that the interpretations have been made in was consistent with the available data" (p. 88). Two confirmability auditors were asked examine the product of the data and provide feedback on the consistency of the available data and the phenomenon interpretations. One auditor, who is knowledgeable of the trans literature and holds a trans identity, examined the data and interpretations related to the trans experiences within the data. The other auditor, who is well-versed in the API literature and holds an API identity, examined the data and interpretations related to the API experiences within the data. Auditors provided general and specific feedback regarding the codes, higher order categories (or meaning units), and themes. They also assisted in differentiating between codes, clarifying definitions of categories and themes, and minimizing double coding. For example, some codes and higher order categories were moved around or renamed/redefined to better encapsulate the participants' experiences, while others were deleted altogether. Each auditors' feedback and suggestions was strongly considered and subsequently incorporated as appropriate.

Bracketing/Reflexivity. Lincoln and Guba's (1985) technique of reflexivity is analogous to Moustakas's (1994) concept of bracketing. The researcher's biases and assumptions about the topic of interest was documented prior to data analysis through a processed known as bracketing (Creswell, 2013; Moustakas, 1994). Examples of potential biases to be bracketed include but are not limited to the researcher's: identities; experiences; knowledge of the available literature related to experiences of racism and transphobia; and expectations of how identity processes are negotiated and experienced. The process of bracketing consisted of the researcher audio recording his assumptions 
and biases, and presenting a summary of these assumptions and biases (see Research section below). Documentation of the detailed, bracketed assumptions made available for the readers ensures transparency of biases throughout the methodological and analytical processes. Bracketing also allows the researcher to be aware of his expectations about the process. An important aspect of phenomenological qualitative research is that the researcher must bracket himself out of the study to ensure that the study is focused on the phenomenon itself and not on the researcher's own experiences and biases (Creswell, 2013; Moustakas, 1994). This process is also known as epoché (Moustaskas, 1994). Bracketing (or epoché) also ensures methodological quality, rigor, and trustworthiness for qualitative analysis (Creswell, 2013; Glesne, 2015; Morrow, 2005; Moustakas, 1994; Wertz, 2005). While bracketing does not completely remove the researcher from the research, it helps prevent the researcher's own experiences and knowledge from being engaged in the process and determining the participants' experiences (Creswell, 2013; Giorgi, 2009; Moustakas, 1994; Wertz, 2005). According to Moustakas (1994):

Although the Epoche is rarely perfectly achieved, the energy, attention, and work involved in reflection and self-dialogue, the intention that underlies the process, and the attitude and frame of reference, significantly reduce the influence of preconceived thoughts, judgments, and biases (p. 90).

Furthermore, it allows readers to be aware of the researcher's experiences so they can determine the extent to which the researcher was able to solely focus and examine the experiences of the participants.

\section{Researcher and Bracketing}

The primary researcher (JT) is a self-identified queer, second-generation Vietnamese American, transman doctoral candidate in counseling psychology. He is also 
an able-bodied person from a middle-class socioeconomic background, who grew up in the South and currently living in West (both in large metropolitan cities). JT's research and social justice advocacy interests focuses on cultural identity development, mental health, and queer and trans experiences, especially those of queer and trans API communities. In a sense, the inception and inspiration for this research was derived from his own experiences as a trans API person living in the U.S. As someone who holds similar identities to the focused population of the study, JT certainly has his own experiences with having to navigate and negotiate cultural values and identities as an API trans man. As a second-generation API individual, he has experienced acculturative stress due to the gaps in acculturation between his second-generation experience and his first-generation family. Specifically, it created conflict in various ways, such as disagreements, misunderstandings, difficulty communicating needs, and struggles to empathizing with each other's experiences. As such, JT's experience as a person of color is directly impacted by immigration and acculturation considerations. Even though he considered himself quite acculturated as a second-generation person, he still holds traditional Vietnamese values (e.g., family is most important).

The areas where JT has lived have been predominantly White. When JT began questioning his gender identity, he wondered if it would be even possible for him to be trans because he had never seen or met another trans API person. He also had a fear that he would be bringing shame to his family by identifying as trans, and subsequently, isolate them from their support systems and community. He felt an intense internal conflict, either to choose himself or his family. He considered making the personal sacrifice to not come out to protect his family. In the end, he had to be true to who he 
was, despite his family reluctance. He felt like his degree of acculturation helped him prioritize his needs and well-being in his negotiation process.

Primary a priori assumptions about trans API people's experiences include: trans API are fully aware of their identity processes, the values they were socialized with, how they navigate spaces, and how they negotiate their identities; trans API people continue to strongly hold collectivistic values but are highly acculturated, which creates internal identity conflicts; conflicts between individualistic trans and collectivistic API cultural values are inevitable; identity conflicts are painful processes; trans API people feel othered in both trans and API spaces; and negotiating identities results in trans API individuals ultimately choosing to come out over their family because their own mental well-being is more important. Some of these assumptions were confirmed in the study's findings, while others demonstrated the contrary. 


\section{CHAPTER IV}

\section{RESULTS}

Data collection produced an overwhelming amount of rich, thick descriptive information regarding these processes. Across the ten interviews, there were eight total themes: Navigating Belongingness (\& Lack Thereof), American Cultural Values, API Cultural Values, Cultural Influences on Gender Identity, Hesitancy, Coming Out, Personal Values, and Integration. Each theme consisted of higher order categories, with some higher order categories comprising of sub-categories. The themes, higher order categories, and sub-categories were described in detail and illustrated using participants' direct quotes. Pseudonyms were used to refer to each participant, with demographic descriptors (age, gender[s], generation, and ethnicity) included using their own words. With participants who stated many various self-descriptors, only one of their descriptors were used (see Table 2 for full participant demographic information).

\section{Theme: Navigating Belongingness (\& Lack Thereof)}

The theme, Navigating Belongingness (\& Lack Thereof), described how trans API individuals experienced the world, including how they were perceived by others. This ultimately affected their processes of experiencing and navigating belongingness and, often, isolation. This theme consisted of six higher order categories (see Table 3): Discrimination, Isolation/Where do I fit in?, On My Own, Not White, Lack of Resources and Representation, and Power of Resources and Representation. 


\section{Table 3}

Navigating Belongingness (\& Lack Thereof) Theme, higher order categories, and sub$\underline{\text { categories. }}$

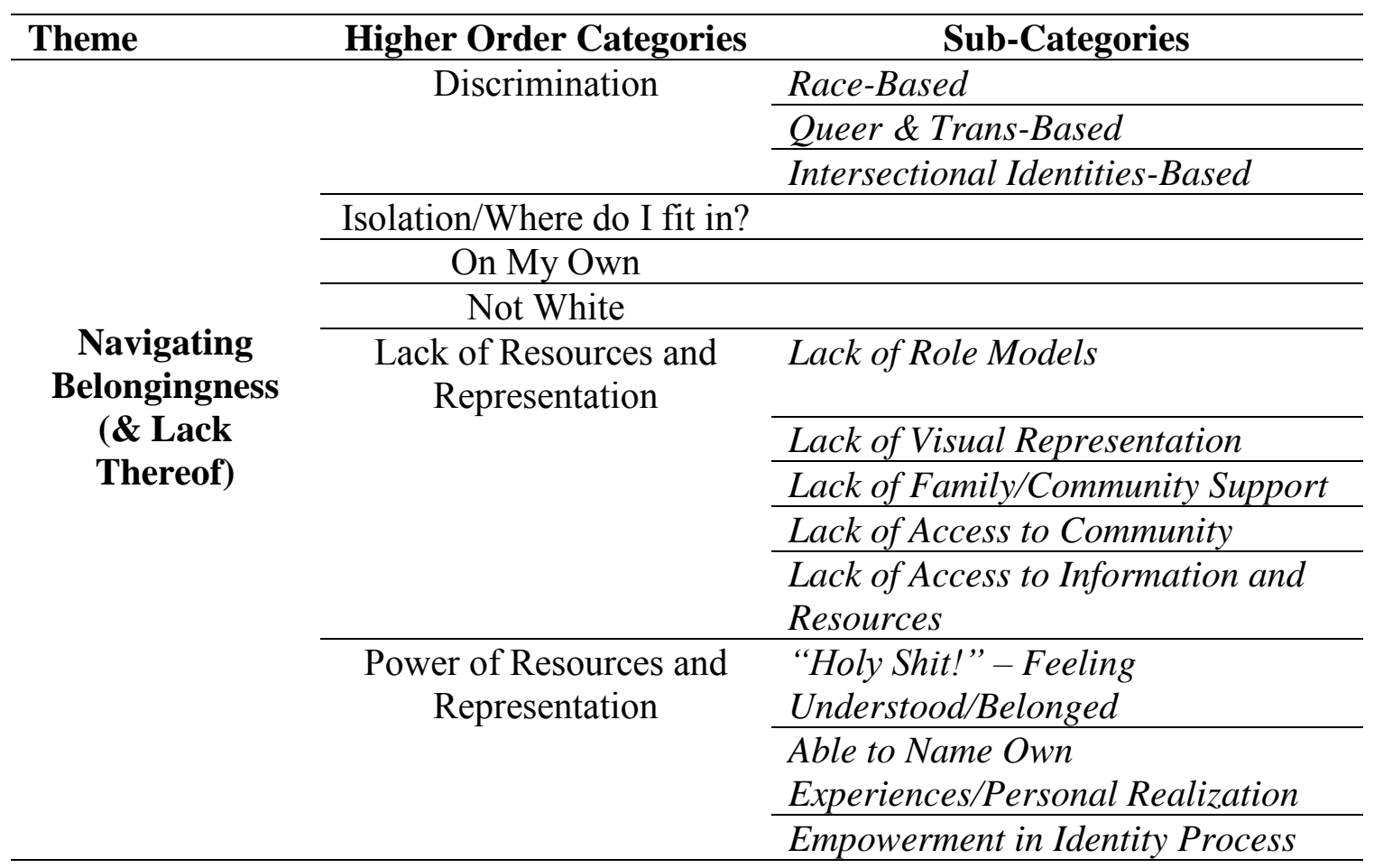

Discrimination. All participants described experiencing some form of discrimination and microaggressions due to their identities. These experiences perpetuated feelings of not belonging, and at worst, feelings of othering. Three subcategories of discrimination and microaggression emerged from the data: Race-based; Queer \& Trans-Based; and Intersectional Identities-Based.

Race-based discrimination. Trans API participants consistently described racebased discrimination and microaggressions throughout their lives. Participants often experienced race-based discrimination and microaggressions from their White peers and community members, both in queer and trans-specific spaces as well as in straight, cisgender environments. Experiences ranged from being tokenized for their race and 
ethnicity to blatant, overt racist pejoratives verbally directed towards them to physical violence.

Tokenization of their race/ethnicity was felt by several participants. The insidiousness of the microaggression left participants often wondering if they were truly being tokenized or if it was all in their heads. This was an experience described by Kai (24, Genderqueer, Taiwanese American, 2nd Generation), who worked at an LGBTQ non-profit organization where they were one of the few API-identifying employees:

It's a lot, it's a lot. Some days, I think about it a lot. And then, at work sometimes it's really hard to focus just because I'm so resentful about being there. Where I'm like "Ugh, am I being tokenized? Or do they really see me?" And also "Am I just letting them treat me like a token?" (Kai, 24, Genderqueer, Taiwanese American, 2nd Generation)

A couple of participants reported experiences of racial slurs during childhood, a developmental time period in which individuals were attempting to form meaningful interpersonal relationships and identities. Carlos (30, Trans man, Filipino, 2nd Generation) shared instances where his API identity was subjected to racial pejoratives:

There have been multiple discrete times in my life that I can think of growing up where I would be in a store or on the playground or something, and kids would be whispering "ching-chong" shit at me basically. And I was like, that's not even how the language sounds [laughs]. That's not even, you're geographically wrong.

(Carlos, 30, Trans man, Filipino, 2nd Generation)

One participant, Mik (42, Nonbinary, Chinese, 1.5 Generation), was even

physically harassed due to being othered as an API person.

So, it was a very hard time. And then at school, I remember being bullied a lot and beat up and made fun of because I didn't speak the language. Called "chink." And I think the cultural aspect was that, being Chinese meant that I was going to be made fun of. (Mik, 42, Nonbinary, Chinese, 1.5 Generation)

For John (24, FTM Trans, Mixed, 2nd Generation), as a biracial API-identified 
individual, race-based discrimination not only came from White spaces but API spaces as well.

I remember growing up in the Korean community, I didn't fit in 'cause everyone there is a hundred percent Korean, and I basically am White to them. So, I never really quite - I always felt like a sore thumb sticking out, and even in traditionally White settings I would get the question of, like, "Do - do you mind...?" asking me "Where are you? What ethnicity are you? Like, where are you from?" No one could quite peg me for who I thought I was. Yeah, I've gotten a lot of guesses.

(John, 24, FTM Trans, Mixed, 2nd Generation)

Queer \& trans-based discrimination. Participants encountered queer \& trans-

based discrimination from mainstream society, as well as within their respective API

communities. In addition to not feeling a part of mainstream society, participants also

felt like they did not belong in API spaces - that they were outsiders to the community in which their racial/ethnic identity was a part. Mik (42, Nonbinary, Chinese, 1.5

Generation) described their experience being in API spaces, "I will get the dirtiest stares and looks."

Intersectional identities-based discrimination. Feelings of being othered and experiences of victimization towards trans API participants' intersectional identities also were described. In particular, Clark (22, Transmasculine, Chinese, 1.5 Generation) shared his negative experiences that ranged from fetishization and tokenization to being perceived as abnormal because of his specific identities as a trans API person. At his place of employment, he explained the difficulty of learning to reconcile and navigate his work environment as a way of survival.

Absolutely. I felt tokenize at work too, at the internship at [place of employment]. I felt kind of fetishized. I felt that my identity became this very, it... my identity became too specific for other people to recognize as normal and ordinary. As a result, my identity became kind of like... maybe remotely Chinese and then, like, I remember there would be like those 
things on the wall where you have ink, it's like bamboo those long kind of things and ju- and just being associated with that. Um... But yeah, I mean it felt like I was struggling to um, administrate my agency in these spaces. (Clark, 22, Transmasculine, Chinese, 1.5 Generation)

Isolation/Where do I fit in? Because trans API participants experienced discrimination due to their identities, all participants reported experiences of isolation and questioning their place in their social contexts. Some participants questioned whether they belong in the API community because of their trans identities.

Because there's always that struggle. Do I belong here? No, I don't. Do I belong in Taiwan? No, I don't. And so, what the fuck does that mean for myself?

(Alex, 33, Genderqueer, Chinese American, 2nd Generation)

Membership within the API community was further complicated by a number of

other factors, in addition to their trans identities. Participants noted how their generational and immigration statuses impacted their relationship to their API community, such that they were often seen as foreigners.

I'm a Chinese person and I actually immigrated to America when I was very young. So that's kind of my ethnic background. As I mainly am Chinese. And I do identify as being a Chinese person but more so as a immigrant... an immigrant Chinese person than I do actually from someone who might be from the mainline and lives there. I do think a lot of the reason I do identify as an immigrant as a qualifier of being Chinese has lots to do with my political alignment. Has a lot to do with my... my understanding that if I were to go back to China I would be seen as a foreigner.

(Clark, 22, Transmasculine, Chinese, 1.5 Generation)

I think, I feel like the sense that I'm a fake, like I'm an imposter within the Indian community.

(Priya, 41, Trans woman, Indian American, 2nd Generation)

Particularly for John (24, FTM Trans, Mixed, 2nd Generation), as a biracial trans API person, he described the White side of identity rendered him as not only a foreigner but as someone who was less-than. 
Whereas I think a lot of second-generation, they're surrounded by that, they're kind of sick of it, they're like, "Okay, I get it, I get it, we're Korean, but I'm American, I want to be American." Whereas for me, I always saw it as, "Oh," there's this world that I so strongly associate with, but even when I'm in it, I feel like I have to prove that I'm Korean enough, that I'm Asian enough to be in it, because I - just by my facial features, I don't fit in. Even though I hold a lot of the same values, a lot of the same experiences, and I'm perhaps - had a little bit more Korean of an experience than some second-generations, like, being kind of relegated into a second citizen, second-class citizen kind of feeling.

(John, 24, FTM Trans, Mixed, 2nd Generation)

Some participants questioned their belongingness in trans and queer spaces

because of being a person of color.

I've interacted with a lot of queer and trans folks and felt like I'm, and this might be my own internalizing of things, but I felt like I'm being judged as less authentic because I'm really willing to sort of speak to a broader sense of things for me. And also because for whatever reason, my body doesn't show up as...people don't necessarily read me as trans right away. Even when I'm binding. Even with the way I present myself. Sometimes I just get read as a queer woman and that could just be because of my body type, because of my height, because of a lot of different things. And that... and also I think because of my brownness, too, if it's a predominantly White trans space.

(Z, 25, Genderqueer, South Asian, 2nd Generation)

Some felt like they just never quite fit in anywhere. Alex's (33, Genderqueer,

Chinese American, 2nd Generation) experience of not knowing where they fit in led them to further existentially questioning their identity, wondering "Who am I?"

Participants expressed frustration and pain that even supportive others did not

understand them and their identity processes. What made sense for the participants and their identity process was confusing for non-API people.

I felt like I needed my family's blessing to do it [begin medical transition], because I also, like, a big thing that I, that's kind of a weird thing to some of my friends. Even though it looks different these days, I tend to nurture my chosen family a little more or my community a little more, I'm still very family and community-oriented. So it's kind of like I - that stuff is really important to me, and that's part of why it took me so long to transition, was 
that I was just kind of, like, I felt like I was betraying them or that I was letting them down or being selfish, even. That even though I was thinking about them and how I going to help them along this whole time, like, because the decision was ultimately mine and one that I decided to pursue, I felt selfish. And I just think that, I remember being very acutely aware at the time of being surrounded by White trans-masculine folks, and not feeling like they really got it.

(Carlos, 30, Trans man, Filipino, 2nd Generation)

It was still different because even if you are doing it the way that you're supposed to, the way that the White mainstream coming-out narrative says it's supposed to be, it's still complicated when you're a brown person. Because I was like, I'm not going to tell my grandfather. Like, he's still alive. He's my last living grandparent. I'm not going to tell him. And even my therapist, who is a Black woman and she's wonderful, she doesn't really get that. She's like, "I don't understand..."

(Z, 25, Genderqueer, South Asian, 2nd Generation)

It was apparent in the data that API and trans spaces, respectively, were not always considered places of comfort, safety, and refuge for participants. Consequently, these feelings of isolation and questions about their belongingness exacerbated negative beliefs about themselves and their identities. Carlos (30, Trans man, Filipino, 2nd Generation) explained, "It [identity process] was a long process of feeling like an anomaly within an anomaly."

On My Own. Participants experienced having to navigate the world and process their identities by themselves. Their experiences of isolation and lack of belongingness created an environment where they had to be self-sufficient, as well as purposeful in creating their own space and accessing resources for themselves. Both Alex (33, Genderqueer, Chinese American, 2nd Generation) and John (24, FTM Trans, Mixed, 2nd Generation) shared this experience of self-reliance during their identity process, due to their intersectional trans API identities and often because of necessity for survival.

Yeah, it's isolating. And I feel like, again, I have to pave my own way. Going back to finding my own identity and not having that parental support. 
There's no support. I have to figure it out for myself. It's what it feels like and it sucks. And so, yeah, it's lonely.

(Alex, 33, Genderqueer, Chinese American, 2nd Generation)

I think it kind of goes hand in hand with the mixed identity because I've never quite truly fit in to one - in one community or another, which means I've kind of always had to, kind of find my own space or create a space for myself where there really wasn't in my community. Just kind of by necessity! Not even a conscious choice, just kind of like, "I have to do this to be able to exist." 'Cause there isn't a space.

(John, 24, FTM Trans, Mixed, 2nd Generation)

One participant noted cultural values influenced her decision-making process of being self-sufficient in her identity process, rather than seeking support from people outside of her family:

I didn't have any kind of ways talking about it, because I was taught not to talk about it. And to talk to my friends seemed disloyal. And it wasn't something that I could talk to my parents about either.

(Priya, 41, Trans woman, Indian American, 2nd Generation)

Not White. Interestingly, several participants noted having the realization that they were not White and their identity process is not that of a White person's. This awareness called into question their belongingness and reinforced their isolation. For some participants, this realization was difficult to accept.

It took me a long time, to - even though these were experiences that I always had, it took me a long time to come to accept the idea that I wasn't White, that I wasn't aspiring to Whiteness or to assimilation.

(Carlos, 30, Trans man, Filipino, 2nd Generation)

I mean for a while, it was like the main...what it [Korean identity] means basically was that I wasn't White. It was the thing that made me not White. (Jaymes, 21, Male, Korean American, 2nd Generation)

Lack of Resources and Representation. Navigating their sense of belongingness and isolation was made difficult because of the lack of resources and representation available to them. Participants consistently specified a lack of and wishes 
for a variety of resources that could have positively facilitated their identity processes as trans API people. These sub-categories indicated in the findings were: Lack of Role Models; Lack of Visual Representation; Lack of Family/Community Support; Lack of Access to Community; and Lack of Access to Information and Resources. These scarcities made navigating the identity processes overwhelming and arduous for participants.

Lack of Role Models. A few participants verbalized having a lack of trans API role models they could turn to for guidance and mentorship. The lack of role models left participants feeling doubt about themselves, questioning their identities, and uncertain of how best to navigate their processes. Before finding other trans API folks or other queer API men to be models for him, RS (34, Transmasculine queer, Taiwanese Chinese, 2nd Generation) explained, "I feel like it was really insecure in how to be. I didn't quite know how to present myself."

These participants wished they had these role models early in their identity process, noting that it would have been supportive and helpful in their negotiation process between their API and trans identities.

I think in the end, the thing that has made it easiest and the thing that the world can always use more of is role models. (Priya, 41, Trans woman, Indian American, 2nd Generation)

Lack of Visual Representation. This was the largest sub-category within this higher order category, meaning there was an overwhelming amount of codes within it. The large amount of data within this sub-category indicated how frequently this topic was brought up, unprompted, throughout the interviews. Thus, it was indicative of the significance of having visual representation for trans API people. Specifically, 
participants shared experiences of not only lacking visual representation of trans API individuals, but also of API people and trans people in general.

Participants noted struggling to see themselves in the media, their surroundings, and in their everyday lives. For many participants, it took them a long time before seeing or meeting a trans person of color, let alone a trans API person. Their introduction to the trans community and being in trans spaces was dominated by Whiteness and White trans people. The Whiteness associated to trans identities often created apprehension in participants' identity process. Z (25, Genderqueer, South Asian, 2nd Generation) described that they questioned their own identity because of the Whiteness associated with trans communities.

And then drunkenly the night of my $21^{\text {st }}$ birthday, "You know, if I really had to, I think I identify as genderqueer, but it's so White, though, I don't know."

(Z, 25, Genderqueer, South Asian, 2nd Generation)

Additionally, nonbinary API participants commented on the centeredness of the gender binary within trans communities and how this impacted their identity processes, which was described by both Kai (24, Genderqueer, Taiwanese American, 2nd Generation) and Mik (42, Nonbinary, Chinese, 1.5 Generation).

I wasn't exposed to queer people or trans people other than in a very binary way... so I think that was why that [nonbinary identity] came later.

(Kai, 24, Genderqueer, Taiwanese American, 2nd Generation)

God, that could've changed my world. I could've probably reached where I feel now being authentic [as a nonbinary person] much sooner, but that's why our generation is different.

(Mik, 42, Nonbinary, Chinese, 1.5 Generation)

Because of the lack of visual representation of trans API individuals and the Whiteness of existing trans spaces, some participants questioned the legitimacy of their 
own identity.

It [White trans spaces] definitely exacerbated the feeling that being trans is a made-up thing by America. It definitely gave some credence to that messaging that I was getting. Obviously I sought out other spaces where there were more people of color, but it was then and continues to be a very White scene...It was hard to see myself, even though a lot of the experience was similar, it was really hard to see myself in other people that I was around at the time.

(Carlos, 30, Trans man, Filipino, 2nd Generation)

Lack of Family/Community Support. While not all participants endorsed a lack of support from their families and communities, those who did described the challenges they face because of this lack of support. Jaymes (21, Male, Korean American, 2nd

Generation) shared his difficulty finding a space that he could feel supported and safe in:

Especially when I wasn't really out at school and I wasn't really out among my friends, it wasn't like there was any sort of safe haven for me anywhere. Even on the internet. (Jaymes, 21, Male, Korean American, 2nd Generation)

Alex (33, Genderqueer, Chinese American, 2nd Generation) specified their API identity as the reason for their struggle to seek and receive support in their identity process:

I get jealous sometimes, but not really. I guess non-Asian POC or more White trans folks who have family members who are in the know or have those conversations. And being Chinese, I don't have those conversations. I don't have that backup. It's a lot tougher, at least for myself, to get that support and not have to do extra steps to do that. I don't know.

(Alex, 33, Genderqueer, Chinese American, 2nd Generation)

Similarly, Kai (24, Genderqueer, Taiwanese American, 2nd Generation)

explained that having family support would have positively impacted their identity process and subsequently, confidence in themselves:

Definitely having family support. That [identity process] would be a lot easier... I guess I would feel less isolated. I, I think it would still be difficult. I think it would still be a lot of work to, to try and come to terms with 
American and Taiwanese values. But, I think having my parents support and encouragement would change a lot. I think I would feel more hopeful. And I would be able to... have more confidence in myself.

(Kai, 24, Genderqueer, Taiwanese American, 2nd Generation)

Lack of Access to Community. Finding trans API community, let alone accessing it, was difficult for participants. In many cases, none existed in proximity of the participants. Similar to the data in Lack of Visual Representation, participants described the existing trans spaces and communities near them as predominantly White in membership. Carlos (30, Trans man, Filipino, 2nd Generation) described, “Obviously, I sought out other spaces where there were more people of color, but it was then and continues to be a very White scene."

As a nonbinary API person, Mik (42, Nonbinary, Chinese, 1.5 Generation) expressed frustration in finding spaces for nonbinary people altogether:

If we don't even have a lot of nonbinary organizations now, we're certainly not going to have Chinese nonbinary organizations.

(Mik, 42, Nonbinary, Chinese, 1.5 Generation)

Because of the lack of family and community support that they had experienced during their identity process, each participant was able to identify the types of support they wished they had. In response to the question what things they wished had been in place to make the process easier of coming to terms with both their API and nonbinary identities, Mik (42, Nonbinary, Chinese, 1.5 Generation) added:

Just more accessibility to resources and people who understand the concept. Maybe just having even our own community be more open and having dialogue and having more maybe groups that you can reach out to. Maybe less segregation. More inclusivity.

(Mik, 42, Nonbinary, Chinese, 1.5 Generation)

Lack of Access to Information \& Resources. The lack of any information or resources associated to their trans API identities made their processes particularly 
difficult and uncertain.

I had so little support around it that I didn't even have language to advocate for myself. I didn't know what I was going through. Really, I had no framework for it.

(Carlos, 30, Trans man, Filipino, 2nd Generation)

One participant attributed the lack of access of information and resources to their

identity as a person of color:

I didn't really know that this is thing as a kid...When I realized, you know, that trans is a word and it meant what I was experiencing, that was when I was about 25 .

(Priya, 41, Trans woman, Indian American, 2nd Generation)

Several participants noted having a lack of access to language or information

about trans identities to even begin to describe and understand their internal experiences, which impacted how they felt about themselves.

So I didn't necessarily have the language to say that it was gender that didn't feel right until very recently, the last 4 or 5 years. And before that, it was always kind of like, there was a part of me that I couldn't really articulate, if that makes sense.

(Z, 25, Genderqueer, South Asian, 2nd Generation)

I think before I had a language for how to identify there were all these feelings I had... These were feelings that made me feel ashamed. Feelings that made me... question whether I had any right to belong. Like, I tried so hard to belong.

(Kai, 24, Genderqueer, Taiwanese American, 2nd Generation)

For Mik (42, Nonbinary, Chinese, 1.5 Generation), the lack of information and

resources hindered their identity and transitional process:

And it was really kind of my own, like, trying to baby-step my way into being comfortable with that. Because I was just never exposed when I was young. So, I didn't know how to go about fulfilling that part of myself..., I spent most of my struggle trying to fit into a community, because I didn't know that transitioning was accessible to me. So I just tried to force myself, like, "Well, then you must be a lesbian." Like, "You must fit into this then." (Mik, 42, Nonbinary, Chinese, 1.5 Generation) 
Power of Resources and Representation. The lack of resources and representation created difficult and isolating experiences for the participants. Thus, when participants were finally able to find resources and representation, it was unsurprising the meaningful and powerful impact they had on their identity processes. Participants identified a variety of resources and sources of representation, including finding/meeting similar others, encountering familial and social support, and accessing information relevant to their experiences. The internet was a common resource amongst the participants. Overarchingly, discovering these resources and representation not only gave participants a sense of comfort, but also feelings of empowerment. Three sub-categories were in this higher order categories: "Holy shit!" - Feeling Understood/Belonged; Able to Name Own Experiences/Personal Realization; and Empowerment in Identity Process.

"Holy shit!" - Feeling Understood/Belonged. Participants expressed feelings of excitement and shock when they were able to find resources and representation of their identities, noting that they finally felt understood and belonged. These resources and representation were not exclusive to trans API identities, but rather the trans community as a whole.

And then once I actually found out that, like, a trans man was a thing, and met the first trans-masculine person I ever met, I was like, "Holy shit!" (Carlos, 30, Trans man, Filipino, 2nd Generation)

These resources and representation facilitated participants' coming out process, both out to others and to themselves. Alex (33, Genderqueer, Chinese American, 2nd Generation) described how having someone share their experience with their identity gave them the courage to also come out:

And it was in college, I was probably 20,21, my best friend had come out as gay and I was like, "Holy crap! I feel like I can come out, too. I'm going 
to piggy back on that a little bit."

(Alex, 33, Genderqueer, Chinese American, 2nd Generation)

Like, I only came to my trans-ness when I met other trans API folks.

(Z, 25, Genderqueer, South Asian, 2nd Generation)

Resources and representation also helped participants not feel othered, which resonated with RS (34, Transmasculine queer, Taiwanese Chinese, 2nd Generation), "I think it helped to meet other trans APIs, because then I didn't feel like a weirdo."

While general trans resources and representation were impactful and significant, trans API resources and representation were even more powerful. Participants noted a sense of familiarity and shared understanding when they encountered other trans API people, particularly with culturally-specific experiences. They felt like they finally belonged somewhere. They felt like they were a part of a community.

The collective is mostly, all South Asian folks. There's a lot of understanding about the kinds of pressure that comes from our families and the kinds of expectations that comes from our parents and the world around us.

(Z, 25, Genderqueer, South Asian, 2nd Generation)

I mean it definitely, there was an instant familial aspect to it, to understand that they were coming from, even though they were, I think they were east Asian, and I'm southeast Asian, and I'm Filipino, so it's, it was definitely the same overarching kind of experience, even if the details weren't exactly the same.

(Carlos, 30, Trans man, Filipino, 2nd Generation)

Additionally, participants described feelings of comfort and freedom in their environments, their situations, and within themselves. Alex (33, Genderqueer, Chinese American, 2nd Generation) described being in POC trans circles, "This is somewhere we can take a breath. Where we don't have to be on edge all the time. We can do it together because we share that same experience."

Resources and representation were not always in the form of other trans API 
people. Participants expressed feeling validated by seeing people who were supportive of trans API loved ones. Carlos (30, Trans man, Filipino, 2nd Generation) explained the impact of seeing a video of a Japanese American mother sharing her love for her trans son and her support of other trans people:

There was that thing going around, and then, so she posted a video that was like, "I love you, and you're valid," you know, saying all the right things, and I remember being like, having my jaw hanging open and being like, "Holy shit!"

(Carlos, 30, Trans man, Filipino, 2nd Generation)

Participants attributed their personal strengths and livelihood to finding these

resources and representation and, ultimately, feeling a sense of belongingness.

It's the fact that it's the community that made me feel like I was a part of something. That I belonged. And so, the triumph of belonging is what makes me have the strength that I have today.

(Mik, 42, Nonbinary, Chinese, 1.5 Generation)

The vitalness of resources and representation was apparent, even if it is not trans API-specific, as explained by Carlos (30, Trans man, Filipino, 2nd Generation) regarding his experience:

Just, not having that community or not - even though the community was largely White and, that community saved my life, knowing that there were other folks out there. Like, walking into a room with thirty trans guys, that literally saved my life.

(Carlos, 30, Trans man, Filipino, 2nd Generation)

Able to Name Own Experiences/Personal Realization. Finding resources and representation was identified as the tipping point in participants' identity processes and opened the floodgates of difficult questions that were crucial to their self-exploration. By observing and learning from similar others, participants were able to name their livedexperiences that they were previously unable to due to lack of information and language.

I mean, I spent a period of time being very, very interested in this dude's [a 
trans man] life, but just as an interest, like, "I'm just so curious about you," and I'm like, "wait, no, tell me everything." And then it just sort of got to the point where I was like, "Oh, wait, I think I am this."

(Carlos, 30, Trans man, Filipino, 2nd Generation)

Like when I was at the Philly Trans Health Conference in 2013 when I met a bunch of folks and that was a hugely eye-opening experience for me to be like, "Oh shit, this thing that I pretended wasn't a thing, is actually very much that. And not only that, there are other people that get it, like I do." (Z, 25, Genderqueer, South Asian, 2nd Generation)

Specifically, trans API resources and representation gave participants the

language and knowledge to understand their own cultural experiences that they were unable to receive in White trans spaces.

The only ways I could name and recognize those things were through seeing other people, more or less, like me. That weren't White. That understood culturally a little bit more about where I was coming from and where my family was coming from.

(Z, 25, Genderqueer, South Asian, 2nd Generation)

Additionally, some participants noted that their identity process would have taken

even longer had they not found resources and representation.

I say it over and over again like how important it was to hear DLo talk. And like, I honestly don't know where I would be had I not been there for like... and I had brought him to campus...And if I hadn't seen that, it would've taken a really long time, I think, for me to even understand the narrative of like, even understand myself as trans, because it...I don't know, sometimes it takes reading or hearing or seeing something who you can relate to, to get your experience. Especially if you're somebody that has been working really hard to be the person that other people really want you to be. I've had to work through a lot of that with my family stuff. (Z, 25, Genderqueer, South Asian, 2nd Generation)

Empowerment in Identity Process. Having resources and representation, particularly of trans API people, empowered participants in their own processes. Kai (24, Genderqueer, Taiwanese American, 2nd Generation) explained that meeting other trans API people allowed them to realize that their identities as a nonbinary person and a 
Taiwanese American person can indeed coexist:

Meeting other trans-people who are Taiwanese... I'm realizing that it is possible to have those two come together, those two parts of me come together.

(Kai, 24, Genderqueer, Taiwanese American, 2nd Generation)

Similarly, Mik (42, Nonbinary, Chinese, 1.5 Generation) was able to realize that

there are many ways and paths to being a trans API person, empowering them to be able

to choose the identities and language that best described their authentic self:

And I think as I got older and I realized that, like, "Wait a second, there's actually more resources available and that I can do some...other avenues." Other paths that actually help me feel more authentic. That's when I started to feel like, "Okay, I can now identify as transmasculine or masculine of center, because that is me."

(Mik, 42, Nonbinary, Chinese, 1.5 Generation)

Having support resources helped facilitate the navigation of spaces and negotiation of identities for many participants. John (24, FTM Trans, Mixed, 2nd Generation) identified “just having a really good support group, honestly" helped him through this negotiation process of synthesizing the API and trans value systems for himself.

\section{Theme: American Cultural Values}

Growing up and living in the United States, there were mainstream American cultural values that participants witnessed and were socialized. This did not necessarily mean participants internalized the messages or continued to hold such cultural values. It was important to note the American cultural values that had the potential to impact how participants navigate their environments and negotiate their trans API identities. Two higher order categories were found (see Table 4): Being an Individual/Individualism; and Status \& Productivity. 


\section{Table 4}

American Cultural Values theme and high order categories.

\begin{tabular}{lc}
\hline Theme & Higher Order Categories \\
\hline American & Being an Individual/Individualism \\
\cline { 2 - 2 } Cultural Values & Status \& Productivity \\
\hline
\end{tabular}

Being an Individual/Individualism. Participants overwhelmingly identified

individualism as a cultural value that was instilled into them by the mainstream American society. They received messages telling them that they are able to, and should be, an individual in society. These cultural messages told them that they get to be an individual and be seen as an individual, rather than as part of a family or community unit. Overall, they were told that it was acceptable, even celebrated, to put their individual selves first over others.

I think the most, the most American thing that I think I instilled was just the sense of like I was an individual. That everything I did was for me and I didn't have to worry about anyone else.

(Clark, 22, Transmasculine, Chinese, 1.5 Generation)

You're not obligated to explain things to anyone. Like, you have no obligations to other people. Or, like, you have more freedom than you think you do. Or, it's okay to be angry at people. Um, also, it's okay to be angry at people and not explain why...It's okay to just be whatever you are. And, you don't have to think about how it's....um... You can let people go from your life. That was a big one.

(Jaymes, 21, Male, Korean American, 2nd Generation)

Interestingly, a couple of participants identified capitalism as a driving force

behind the American values that participants witnessed, as noted by both RS (34,

Transmasculine queer, Taiwanese Chinese, 2nd Generation) and Kai (24, Genderqueer, Taiwanese American, 2nd Generation).

So, I feel like, you know, having a cultural value in some way centers family as opposed to against the American values, especially with the 
capitalism and everything, to be an individual, individualistic, and be more self-involved. And I feel like, those sometimes, those things kind of conflict, because as a Chinese person, at the end of the day, I feel like relationships are more heavily valued still.

(RS, 34, Transmasculine queer, Taiwanese Chinese, 2nd Generation)

Being an individual, which is in a western sense it seems possible you can be your own person you can define yourself for yourself ... That's a really capitalist way of thinking, like you can just be an individual and everything you do is kind of in it's own kind of bubble. (Kai, 24, Genderqueer, Taiwanese American, 2nd Generation)

Status \& Productivity. In addition to individualism as a staple of American cultural values, participants also described cultural values related to having status (e.g., merit, prestige, meeting the status quo) and having one's worth in society be measured by their productivity (e.g., amount of work done, participating in capitalism, pulling oneself up by the bootstrap).

I mean, definitely success and achievement being closely intertwined. That being a productive member of society and participating in capitalism is what you need to do to be a person of value. And that you always have to keep trying to be doing more. That that's something that people respect and that's something that people value, and that's like - if you're not able to achieve a certain amount, then you are seen as less than. So there's that. And definitely having that closely tied to, like, well, if you are poor, and you are not trying to be not poor, then you are lazy and stupid and whatever, or a bad person.

(Carlos, 30, Trans man, Filipino, 2nd Generation)

\section{Theme: API Cultural Values}

Similar to American cultural values, being raised in API families and communities also presented ample opportunities to observe and receive cultural values from their family and culture of origin. While these values also did not imply automatic internalization of these messages, they had the potential to shape the experiences and ways in which participants navigate their surroundings and negotiate their identities. There were seven high order categories within this theme, each an identified API cultural 
value (see Table 5): Take Care of One's Family; Educational \& Status Attainment via Work Ethics; Keep it to Yourself; For the Community; Filial Piety; Saving Face; and Community Closeness.

\section{Table 5}

API Cultural Values theme and high order categories.

\begin{tabular}{|c|c|}
\hline Theme & Higher Order Categories \\
\hline \multirow{7}{*}{$\begin{array}{l}\text { API Cultural } \\
\text { Values }\end{array}$} & Take Care of One's Family \\
\hline & Educational \& Status Attainment via Work Ethics \\
\hline & Keep it to Yourself \\
\hline & For the Community \\
\hline & Filial Piety \\
\hline & Saving Face \\
\hline & Community Closeness \\
\hline
\end{tabular}

Take Care of One's Family. As the largest one under the current theme, this higher order category was defined by the cultural value that emphasized the responsibility of individuals putting one's family above all else, including oneself. Such responsibilities included making personal sacrifices and ignoring personal needs for the greater good of the family. This was clearly described by Kai (24, Genderqueer, Taiwanese American, 2nd Generation), "Many immigrant, specifically East Asian families... it's family first, over being an individual, over having your own agency and self-determination." This message was the opposite of Individualism found in the American Cultural Values theme, which called for putting individual's needs in the forefront.

The biggest one [cultural value] I think is that family comes first. Like, that's been the most important thing. I've never questioned for a second that if my parents want me home, I'm coming home. If my mom calls me, I have to call her back. If my sister needs something, I'm there before I'm there for anybody else. Those things are all, like, family is the most important thing and the clearest value throughout my whole life. 


\section{(Z, 25, Genderqueer, South Asian, 2nd Generation)}

Educational \& Status Attainment via Work Ethics. Participants shared having expectations placed upon them by their families and communities that called for them to work hard to achieve educational attainment and status. Status attainment was often described as being in the form of achievement and success, as well as social standing via career choice. Clark (22, Transmasculine, Chinese, 1.5 Generation) clearly expressed how API cultural values instilled into him "has to do with valuing education as this kind of pinnacle of achievement." Having a strong work ethic at all times, across all domains, was necessary and expected by participants.

I think another one is very much about work ethic and like, trying to succeed in what you're doing at all times. And working hard and working, like um,...yeah. And centering work in a way that feels...yeah, that allows for mastery and success, but not necessarily in the....no, I mean like, unfortunately, in sort of that prestige sense, too...But the value that was always instilled in me was sort of be the best at your job. Be the best at what you're doing.

(Z, 25, Genderqueer, South Asian, 2nd Generation)

Keep it to Yourself. Regardless of the event or emotion, participants were expected to remain stoic and in control of their own emotions. Within families, members were expected to keep their feelings and experiences to themselves, often as a personal sacrifice. As a family unit, families were further expected to keep their familial struggles out of the community, in such a way that Priya (41, Trans woman, Indian American, 2nd Generation) described as, "We don't really talk about our feelings. We don't really....uh, we don't air our dirty laundry outside the house. We don't do it that much inside the house either." Alex (33, Genderqueer, Chinese American, 2nd Generation) echoed her experiences:

Oh yeah, you keep it tight and just don't talk about it. I always remember 
this story. I was probably, like, between 11 and 13, and I was fighting with my dad and I just started tearing up and he's just, "You don't cry! Don't show your emotions." I'm like, "What the hell? Why can't I show my emotions?"

(Alex, 33, Genderqueer, Chinese American, 2nd Generation)

For the Community. In addition to family being vitally important, participants also identified the cultural value that also saw API community of origin as central. The community of origin was important, such that one must give back and hold true to it.

That you, you do not belong to yourself as much as you do the community that you, you'd grown up with that have given up also so much for you. (Clark, 22, Transmasculine, Chinese, 1.5 Generation)

I'd also say a strong sense of doing good for your community and being connected, to people who are part of either your family or your chosen family, was a huge thing.

(John, 24, FTM Trans, Mixed, 2nd Generation)

Filial Piety. Participants identified the existence of clear expectations and responsibilities placed upon them as it relates to respect and deference, particularly towards elders and those in higher statuses.

A lot of deference to elders. I was actually super shy, so other than being able to act within knowing where my place was within the hierarchy, social hierarchy, I didn't really know how to be myself, I just knew, like, "Oh, I'm older than this person, so I am, like, you know, their elder, they should listen to me," or, "Oh, this person is elder than me, I need to defer to them," was a huge thing. (John, 24, FTM Trans, Mixed, 2nd Generation)

Respect and deference also encompassed the expectation of following the status quo set forth by elders and the community.

It was a very much top-down kind of situation of like, we're gonna tell you what to do and you're gonna do it, and that's, you know, there wasn't really much room for, like, conversation going both ways... I think that not even just with regard to gender and sexuality stuff but I feel like so much of Filipino culture is transcribed and laid out for you already the way that like it's very clear what you should be doing or what you should be working on, how you should have a family, how you should date people. 
(Carlos, 30, Trans man, Filipino, 2nd Generation)

Saving Face. Participants described expectations that they must maintain face for themselves, their families, and others. When participants discussed the idea of saving face, they often mentioned it by name. Saving face was also described as being polite and nice to people, as noted by Jaymes (21, Male, Korean American, 2nd Generation). Saving face served to protect the family, as well as the family's honor and dignity. Participants often described instances in which they had to hide themselves and their identities to maintain an external perception for their families, as explained by Kai (24, Genderqueer, Taiwanese American, 2nd Generation).

Definitely saving face... So when I got the job that I currently have, this was a little after I came out. I had already come out and so I took this job and I told her this is where I'm going to work and she said, "I have no problem with you being gay in your private life but don't make it public because everyone in family is going to know. People are going to know and that's shameful."

(Kai, 24, Genderqueer, Taiwanese American, 2nd Generation)

Community Closeness. Coming from an immigrant family and community came with the expectation that people stuck close to their communities of origin, which was reported by a few participants. Families transmitting the importance of sticking close to communities of origin, in a way, created an atmosphere of either mistrust of outside communities or pressure to remain insular. The ways in which participants were expected to stick close to their community varied. Growing up in an Indian household, Priya (41, Trans woman, Indian American, 2nd Generation) stated that her parents wanted her to marry an Indian person. While Clark (22, Transmasculine, Chinese, 1.5 Generation) described his experience differently:

My parents are very... I think very conservative people. They have a view of themselves as being... as kind of existing in this... they have this fort 
that they just built up, and White people and people of different ethnicities fall on the other side, and there's this very close knit group of very sheltered immigrants that only they can trust. And so... from a very young age, I got the sense of there's us versus them mentality. (Clark, 22, Transmasculine, Chinese, 1.5 Generation)

\section{Theme: Cultural Influences on Gender Identity}

Culture, from their culture of origin and mainstream America, appeared to have some influence in the overall gender identity process for participants. In some cases, participants expressed how cultural values informed their process, while other cases were related to cultural experiences and environments. Some values were inherently gendered, while others were not related to gender at all yet they still influenced their gender identity process. These experiences and values were socialized and internalized by participants, which impacted their experiences with their gender identity, transition process, and view of self. Four higher order categories were a part of this theme (see Table 6): API Family/Community Cultural Influences; Mainstream American Cultural Influences; Gendered Cultural Expectations; and Acculturation.

\section{Table 6}

Cultural Influences on Gender Identity theme and higher order categories.

\begin{tabular}{cc}
\hline Theme & Higher Order Categories \\
\hline Cultural & API Family/Community Cultural Influences \\
\cline { 2 - 2 } Influences on & Mainstream American Cultural Influences \\
\cline { 2 - 2 } Gender Identity & Gendered Cultural Expectations \\
\cline { 2 - 2 } & Acculturation \\
\hline
\end{tabular}

API Family/Community Cultural Influences. Participants described a number of API cultural experiences and values that impacted their gender identity process. Rather than naming cultural values that influenced how he experienced his transition process, Jaymes (21, Male, Korean American, 2nd Generation) described being in API 
environments that allowed him to gain more comfort in his body as he transitioned:

I mean, that was one of the things that was kind of helpful in the beginning, because it was nice to know that...that...so I went to a high school that was $70 \%$ Asian. So, I saw a lot of Asian teenage boys. So, like, I had a good sense of what was kind of the deal...There was...I mean, there's more room for me with a baby face than me with no facial hair. Like, that was going to be kind of fine. So, in a way, it felt like a little happy gift that I wasn't going to have to....like, I didn't have to feel shitty about not having much facial hair or having any facial hair, because neither did my dad. I didn't need to obsess about how tall I was going to be, necessarily, because neither was my dad. And neither was any other 14-year-old Asian boy around me. (Jaymes, 21, Male, Korean American, 2nd Generation)

On the other hand, cultural environment also created struggle in the process. Kai (24, Genderqueer, Taiwanese American, 2nd Generation) explained how their cultural environment and values, comparing themselves to other Taiwanese people, created a struggle for them to understand their own body:

I think it really changed my relationship to myself. Looking at myself and feeling my own body. I remember I had an eating disorder from age 13 to 20 and... And I remember wondering how can I, as a 5'5 Asian person, ever look like every other Taiwanese person. Because I remember wanting, wanting so badly to look like my mom and to look like my sister... and feeling this pressure to be pretty, so I could have a boyfriend basically and get married and not live alone... So I think it was very, I think internalizing so much of the messages of gender, even the implicit messages about gender and race and nationality, it was damaging for me because I didn't know my own body. (Kai, 24, Genderqueer, Taiwanese American, 2nd Generation)

Similarly, Clark (22, Transmasculine, Chinese, 1.5 Generation) shared how his internalized cultural values associated with family kept him from raising questions and exploring their identity:

And I think growing up I was very, very against these kind of, these ideas of expressing yourself like, you know journaling, meditating, being more open with yourself. I was much - I was like so, so repressed. And I also attach a lot of repression with being Chinese and it's because... because there's so much fear I think in the culture of asking questions and 
receiving this answer that would just blow up your life. Which I think in many ways, realizing that I was transgender did.

(Clark, 22, Transmasculine, Chinese, 1.5 Generation)

Priya (41, Trans woman, Indian American, 2nd Generation) specifically noted her religious upbringing, describing how it empowered her as she explored herself and reached a place of self-understanding.

I think actually, the thing that I keep, I try to keep in my mind is, um, is actually a very Indian thing. So, in the Bhagavad Gita, Krishna, who is my family's namesake, is an incarnation of Vishnu. And he, he makes himself into an Arjuna charioteer and counselor. And he stays with Arjuna and his family through the conflict. Bhagavad Gita is this conversation, essentially, between Krishna and Arjuna. And it's very much like the Book of Job in a way, because basically, Arjuna's position is that he can't make decisions that he can't deal with the possibility of having to harm people or cause pain or inflict violence. And Krishna's central answer to Arjuna is in essence that, what we do is not try to memorize the right and wrong answers to specific situations, and that doesn't work. But what we do instead is we invest in being right-minded and in building our heart, our empathy, our mind, our consciousness, our values. The idea is that there's a going to some source of authority, because what Arjuna wanted was for someone to tell him, "It's okay for you to fight this war." But that doesn't go to some source of authority, we become the source of authority, because we've been invested in rightmindedness. We can rely on ourselves to make good decisions. We can rely on ourselves to be a source of righteous and virtue in difficult situations. And so, that's what I keep in my mind. That as I'm navigating all of this, I have finally reached a place where I have a sense of who I am on multiple levels.

(Priya, 41, Trans woman, Indian American, 2nd Generation)

Mainstream American Cultural Influences. Similar to the previous higher

order category, participants also shared how mainstream American cultural experiences and values influenced their gender identity processes. Participants overwhelmingly mentioned individualism as the key that allowed them to explore and express themselves. They noted the openness they had in taking steps to begin asking questions about their gender identities and ultimately, to come out. 
Because it allowed that openness to come out, to explore that, instead of trying to follow my parents' values of meet a guy, go to school, meet somebody, get married, have kids, you know, that type of thing. And so, you know, just, there's that openness to explore myself.

(Alex, 33, Genderqueer, Chinese American, 2nd Generation)

Part of individualism also came with self-advocacy and living for oneself rather

than for others. Individualism gave participants permission to pursue their social and medical transitional process, which was described by both Z (25, Genderqueer, South

Asian, 2nd Generation) and Carlos (30, Trans man, Filipino, 2nd Generation).

To put my foot down and say, "I want this surgery. And no, I want to use these pronouns. I want you to use this name." Those kinds of things.

That has been new for my family.

(Z, 25, Genderqueer, South Asian, 2nd Generation)

Well, I mean, I have to thank American culture for telling me it was okay to live for myself, for sure. That is the element of Americanism that I feel like had a big part of my trans identity that is like, "Okay, this is okay for you to want for yourself and for you to act on for yourself," because it felt revolutionary to me to have people tell me that, you know, "At the end of the day, you're the person that has to live in your body, not your mom, not your sister, not your aunts," and that felt so far removed from the messaging that I got when I grew up.

(Carlos, 30, Trans man, Filipino, 2nd Generation)

Some participants noted how American culture had more flexibility in what was considered "okay" or acceptable, as noted by Priya (41, Trans woman, Indian American, 2nd Generation):

Yeah, I mean, I think it definitely sort of leveraged the fact that America has, in some ways, a broader kind of sense of what is okay. (Priya, 41, Trans woman, Indian American, 2nd Generation)

For Jaymes (21, Male, Korean American, 2nd Generation), American culture, particularly predominantly White American trans culture, contributed to a feeling of pressure to fulfill his gender identity in a certain way.

Well, just being in that, being in those spaces taught me that the way to 
win is to be really hot and the way to win is to be conventionally attractive and skinny but also fit, but also wear the right clothes and do this and do that. But it also taught me, and you also have to be a little different. And you have to not look like a trans guy. Like, basically, that's what it boils down to. You have to not look trans. You have to look super impressive, meaning that people will go, "I had no idea!" Like, that was the gold standard of like...that was, like, my dream. Like, I never really totally admitted it to myself, but I was kind of obsessed with that....I get really worked up about, or at least I used to, I don't know, like, “Oh, people should...I should wear the tightest shirt I can so that people will know that I've had top surgery." So that they know that I'm not binding or assume that I have boobs, or something. Because I don't want them to think about that.

(Jaymes, 21, Male, Korean American, 2nd Generation)

Gendered Cultural Expectations. There were experiences and values from both cultures that were inherently gendered. It was important to note the role of socialized gendered messages, particularly as they relate to participants' experiences with their own genders and gender identity processes. The most common gendered cultural influences mentioned by participants were the API and American socially-imposed cultural expectations for men and women, such as gender roles and what masculinity/femininity should look. Participants noted various ways in which these expectations impacted them. For example, there were gender role expectations based on participants' assigned sex at birth. This influenced the ways participants were able to or not able to express themselves in certain ways.

I know the reason why I am a sensitive person and the fact that it was nurtured in me in a lot of ways, was because I was a female-identified person. Like, people saw me as a girl and they let me cry and they let me say like, "My feelings are hurt." And they let me talk through those things. If I had been read as male, that wouldn't be the case. (Z, 25, Genderqueer, South Asian, 2nd Generation)

Z (25, Genderqueer, South Asian, 2nd Generation) went on to describe how bodies and body shapes were also gendered in their culture: 
I think the hair thing was really huge. So, a lot of South Asian women, um, the expectation is long, beautiful hair and you care for it and it's beautiful and thick. Cutting is sort of like a crime against your culture in some ways. So deciding to cut it was culturally important...Y Yeah, and I think, too, like body type of generally South Asian women is supposed to be curvy and like...not that you're supposed to show off your curves in any particular way, but also wanting to actively go against that is, um, was something that I sort of had to think about and how much of that was sort of tied to my physical sense of self and the expectations for what I was supposed to look like. How my body was supposed to fit with ideas of a woman or as a person.

(Z, 25, Genderqueer, South Asian, 2nd Generation)

These expectations also informed participants of how they should perform their

current gender(s). Jaymes (21, Male, Korean American, 2nd Generation) noted how

these expectations gave him ideas of how to perform his male gender.

It's just a general role that I feel okay being a part of. Or a collection of cultural benchmarks against which I, like, maybe not, well, measure myself, but also use to guide myself... Well, things like dress sense. Things like, not necessarily specifically what clothes, but what sort of like, shape. Sort of like general overall aesthetic and visual sort of, there's the silhouette, general color scheme, and things. Oh my God. Just like, uh, the general look that I think that's one of...uh, things that I think about more. Also, and I mean, a lot of these benchmarks are just sort of culturally things, aren't necessarily things that I use to be like, "Oh, I got to be that." But more like, "Oh, that's what I'm aware of and that is shaping how I'm behaving." So, like, a lot of emotional things and how guys talk and how guys...the way that they're supposed to talk and ways that they end up talking. And like, topics of conversations, too. Because I have been aware of...like, a part of being a guy to me is to be aware of what are expected ways of talking as a man.

(Jaymes, 21, Male, Korean American, 2nd Generation)

Contrarily, Clark (22, Transmasculine, Chinese, 1.5 Generation) detailed how he

felt pressured to perform and express his gender to meet cultural expectations, even as he

questioned whether these expectations aligned with the type of person he wanted to be.

As I was transitioning, my American friends would compliment me more when I was performing more Western ideals, like when I was being more overtly masculine and making like very kind of... there's these type of offensive frat boy jokes that are made, know what I mean? Like so there 
was this reward for performing masculinity as this kind of oppressive thing. So, I began to associate my transness with being like this douchy person, know what I mean? The way in which, like men kind of like causally joke about women. I was like internalizing a lot of that and trying to question if, whether or not I was replacing ideals of femininity with ideals of masculinity. And, and that became really uncomfortable for me as a trans person... So, um I learned that I had to really question these ideals of Western masculinity that I was taught for so long. And um, and a lot of it had to do with not taking out my failed masculinity on say, women or making jokes about other people and not to make my transition other people's problem...I felt like this is what I have to do to be a man? This is what I have to do to be a Western American man? Like, I have to, I have to be causally misogynistic, casually just dismissive of other people's emotions, I have to just call people crazy because they might have gotten frustrated, you know what I mean? I feel like I was fitting into a role that was very Western, very American. And I didn't, in this sense the qualities of individualism, self-sufficiency and performativity all became very strong negatives to me and I wanted to absolve myself with any sort of Western-ness in my trans-identity. (Clark, 22, Transmasculine, Chinese, 1.5 Generation)

Additionally, participants noted gendered cultural expectations also helped them

realize their own gender(s) and who they wanted to be.

I don't think I could stand up to the values and expectations of being a woman, both as a Chinese and as American in two different ways...Like, my mom was a, basically a full-time mom, full-time home care person and that was a lot of work and I had no interest in participating in any of that much. So, the cooking, taking care of other people, necessarily in the nurturing, in that specific way. I didn't know how to do in that style. And in American values, I couldn't....I also couldn't play into that role. Well, actually, I did pretty well actually now that I think about it. Um, but uh, dressing pretty and that kind of stuff, I couldn't do...Um, I knew that I couldn't...um, I couldn't meet those kinds of expectations if I had to be a girl because I would...I would do a crappy job.

(RS, 34, Transmasculine queer, Taiwanese Chinese, 2nd Generation)

Furthermore, one participant identified the socially-imposed gender binary as a

possible reason why he identified as a binary gender.

So like - I have some weird, like, binary trans guy guilt about it, 'cause you know, the kids these days and all their genders, I just kind of, I wonder sometimes about myself, and whether or not I would feel so binary identified if I wasn't so strictly raised in a gender binary. It's 
definitely something I wonder to myself. And I definitely see that as a function of my culture and of being Filipino.

(Carlos, 30, Trans man, Filipino, 2nd Generation)

Acculturation. The last higher order category of this theme was related to how much participants acculturated into mainstream American society. The degree of acculturation appeared to affect how much individualism or family-focus values influenced some participants' gender identity processes. These participants described feeling acculturated, "Western," and "Americanized." They generally identified more with a sense of individualism and other experiences associated with mainstream American culture.

Um, I am kind of a coconut. I am very acculturated as an American and I have been for a very long time.

(Priya, 41, Trans woman, Indian American, 2nd Generation)

I also think that I am much more American than Chinese in some ways, because my own...my own socializing values tend to be more American. So more individualistic.

(RS, 34, Transmasculine queer, Taiwanese Chinese, 2nd Generation)

So, I think in that way I'm more Americanized. I have this idea of individualism as opposed to this kind of social community that we sacrifice and... and endure for.

(Clark, 22, Transmasculine, Chinese, 1.5 Generation)

Kai (24, Genderqueer, Taiwanese American, 2nd Generation) mentioned their

degree of acculturation was the product of being a second generation Taiwanese

American:

Being more family oriented is a value that again, I didn't really feel strongly about. Partly because of being born in the U.S. and being raised here.

(Kai, 24, Genderqueer, Taiwanese American, 2nd Generation)

On the other hand, Mik (42, Nonbinary, Chinese, 1.5 Generation) described acculturation and assimilation as a form of survival as an immigrant: 
But I think that, what it might maybe comes down to was in my childhood, I had to assimilate a lot. I had to assimilate and fit in. Like, forced to fit in somewhere... But I think what it does is after a long period of time in your childhood, I always had to, I was always placed in these situations where I had to assimilate and fit in and belong, because I never quite belong anywhere, that maybe as a young person I felt very insincere. Because when you assimilate, often times you don't get to choose who you are, you just have to be to fit in. And I think that having gone...I went through some trauma when I was young. (Mik, 42, Nonbinary, Chinese, 1.5 Generation)

\section{Theme: Hesitancy}

This theme described reasons why participants were hesitant to come out to themselves, their families, and others about their gender identity. Participants shared experiences of having to navigate environments and negotiate their cultural values to determine the course of action as they attempted to explore their gender identities. In many cases, part of the course of action was questioning whether to come out as trans and gender nonbinary individuals. For many participants, there was hesitancy in that process.

This theme consisted of six higher order categories (see Table 7): Family First;

Interpersonal Interactions/Reactions; Uncertainty; Lack of Language/Communication; Self, First; and Internalized Messages.

\section{Table 7}

Hesitancy theme and higher order categories.

\begin{tabular}{ll}
\hline Theme & Higher Order Categories \\
\hline \multirow{3}{*}{ Hesitancy } & Family First \\
\cline { 2 - 2 } & \multicolumn{1}{c}{ Interpersonal Interactions/Reactions } \\
\cline { 2 - 2 } & Lack of Language/Communication \\
\cline { 2 - 2 } & Self, First \\
\hline
\end{tabular}

Family First. For most participants, the hesitation to come out stemmed from 
wanting to put their family first, often worrying about the impact their coming out might have on their family and their family dynamics. Priya (41, Trans woman, Indian American, 2nd Generation) questioned, "How would [coming out] affect the family?" Other participants had already felt they found the answer to that question, such that they would negatively affect the family.

You know, in many ways I thought of my being trans as a being a burden on them. You know, it was like another, it was a thing that I would drop on them that, that would really disrupt our relationship. (Clark, 22, Transmasculine, Chinese, 1.5 Generation)

I just felt like I was constantly embarrassing her, and constantly making her feel shame.

(Carlos, 30, Trans man, Filipino, 2nd Generation)

Some participants identified their families as the reason why they prolonged their coming out and/or postponed their transition process.

I mean, my dad was really big into family. He was kind of like, actually he was always on my case in my childhood about prioritizing friends over family, wanting to go do things with my friends and wanting to skip family things, or do holidays with other families, that kind of thing. And he was always like, "Your family is the most important, and you always have to put your family first, and you know that your friends come and go but your family is always there." And so that was always a really important element to me - and still. Even though it looks different these days, I tend to nurture my chosen family a little more or my community a little more, I'm still very family and community-oriented. So, it's kind of like I - that stuff is really important to me, and that's part of why it took me so long to transition, was that I was just kind of, like, I felt like I was betraying them or that I was letting them down or being selfish, even. That even though I was thinking about them and how I going to help them along this whole time, like, because the decision was ultimately mine and one that I decided to pursue, I felt selfish...I was sort of stuck for two years after I came out, where I was afraid to make the wrong move or afraid to disappoint people or alienate my family. So, having that going on my head in sort of an anxiety freeze.

(Carlos, 30, Trans man, Filipino, 2nd Generation)

The desire to protect the family was strong for many. For Priya (41, Trans 
woman, Indian American, 2nd Generation), she even considered waiting it out until her

parents both passed.

I didn't know if my parents could take it. And so I thought to myself, "Well, maybe I would give this some time when my parents were gone." And, you know, I hoped that that was a long time away. And the downside of that was, of course, that I would be 50 years, 60 years and missing out on my life all that time.

(Priya, 41, Trans woman, Indian American, 2nd Generation)

In RS's (34, Transmasculine queer, Taiwanese Chinese, 2nd Generation) case, he initially came to the conclusion to sacrifice his own person well-being (e.g., coming out) for the well-being of his family.

So that, when it comes to being trans, I would negotiate somewhat about what that means, which is I would not necessarily, like, I would actually try and maybe compromise. Before in the past, how I would be presenting myself just to appease my, just to make it less confrontational with my family. But also, that it would be better for me to just be, you know, instead of like, coming out and maybe then, in some cases, people just break up and stop being family with each other. That's less of an acceptable thing. (RS, 34, Transmasculine queer, Taiwanese Chinese, 2nd Generation)

Interpersonal Interaction/Reactions. Participants had fears related to how

others, particularly their families, would react to their gender identities if they did come out. Fear of reactions varied from nonacceptance to even hostility.

I've been hesitating because I have no idea how she'll take it...It's really hard for me to keep secrets from someone, and especially feeling like I'm hiding a part of myself from someone who's very close and important to me. A bit painful. But at the same time it's like, I still rely on her a little financially, so I almost have to balance between what I want, what my heart says to do, and what my brain says to do, which is like, if worse comes to worse and she doesn't accept you, and she withdraws her support because of her beliefs, then it's better to kind of hide that until you're truly independent, but that also makes me feel kind of frustrated at the same time.

(John, 24, FTM Trans, Mixed, 2nd Generation)

I thought to myself, "Well, if worse comes to worse, I'll sit on a curb and 
call for a cab." But even though I didn't think my parents would react that way, I had to be ready for that.

(Priya, 41, Trans woman, Indian American, 2nd Generation)

Kai (24, Genderqueer, Taiwanese American, 2nd Generation) noted their negative experience in coming out to their parents as queer previously informed their decision to not come out as genderqueer.

When I came out as queer it was that kind of extreme where my mom was like "You chose this and that's wrong. It'd be okay if you were born this way but I know you weren't born this way." So definitely if I came out as trans it would be that similar understanding where there is right or wrong... and if you're wrong then you deserve to be punished, you deserve to be unhappy.

(Kai, 24, Genderqueer, Taiwanese American, 2nd Generation)

Uncertainty. A few participants felt uncertainty about what it was that they were experiencing and what the coming out/transition process would even entail for themselves. The uncertainty fostered fear in the participants, which became debilitating at times. For John (24, FTM Trans, Mixed, 2nd Generation), he felt trapped prior to coming out, while also acknowledging a sense of comfort and familiarity in that space:

I felt like I was trapped in a box before, and now, while - it's honestly super scary to go out of that box, because I'm very familiar with that box, but it also didn't allow me to grow as a person... I haven't really - I've kind of been taking baby steps this entire time, so, while I've considered physical changes like chest binding and things, I don't think I'm quite ready for that.

(John, 24, FTM Trans, Mixed, 2nd Generation)

Some participants stated the unfamiliarity of the transition process contributed to the sense of fear.

At the time I had phrased it to myself, I had rationalized it to myself as a, "I'm just, you know, making sure and making the right decision, and I'm biding my time, and I'm considering all options," and stuff, but I was really just afraid of what would happen.

(Carlos, 30, Trans man, Filipino, 2nd Generation) 
Lack of Language/Communication. Participants identified the lack of language and communication abilities to even verbalize their identities, let alone their transitional and emotional experiences. Some participants described not knowing how to talk about their gender identity, in general. Some explained that the language barrier between them and their families prevented them from explaining their experiences in a way that was understood.

I think the language barrier was an issue, of trying to talk to me, "cause I used to speak Korean with her, but she stopped speaking Korean with me so she could improve her English, and she was the only person I spoke Korean with, so I lost those skills... And I can't even - it's kind of been like a lifelong frustration because I can't even talk to my family. Like - I mean, not even about gender identity, but about emotions, on my Korean side, so. [laughs] (John, 24, FTM Trans, Mixed, 2nd Generation)

These language barriers were often due to losing language skills over time or were nonexistent altogether. For Kai (24, Genderqueer, Taiwanese American, 2nd Generation), they expressed feelings of guilt because of it:

I often don't tell people that I am Taiwanese 'cause I still feel guilty about it. Guilty for being Taiwanese but also not speaking it and not being able to read it... not being able to communicate to anyone in Mandarin. (Kai, 24, Genderqueer, Taiwanese American, 2nd Generation)

Self, First. This higher order category described putting oneself first by not coming out to the family. While only one participant made up this category, it was particularly important to report. It challenged the hegemonic notion that putting the family first is the only reason to not come out. For Kai (24, Genderqueer, Taiwanese American, 2nd Generation), putting themselves first was their reason to not come out to their family and it was their way of navigating their environment. In their situation, it meant running away to not have to come out to their family and it was for survival. 
I think identity isn't as meaningful as being able to survive, essentially. And I wish I would explain it to them as, "You know, this is actually how I am surviving. These relationships help me survive a lot." And that's why I ran away, because I wanted to be with my partners. And it was just not, it was so toxic, and it was not going to help if I stayed at home. Even when I didn't have to pay rent. So, ultimately, I decided not to come out as trans.

(Kai, 24, Genderqueer, Taiwanese American, 2nd Generation)

Internalized Messages. There were negative internalized messages that caused

fears or hesitation in participants' identity and coming out process. These messages came from family, mainstream society, culture, and even the trans community. Some participants internalized negatives messages, such as shame and hatred, which impacted how they saw and understood themselves.

And so, in that sense my, my very conservative parents also have this you know, I mean, they're also very openly homophobic and trans-phobic and xenophobic so I think a lot of that I internalized that and was like "I can't possibly think abut gender right now cause that's gay and I'm not gay" so it was like, it was, it was internalizing their hatred and their shame as well. And I think a lot of their shame comes from this fact that we kind of, can't assimilate into American culture but we can adopt casual homophobia and, and... the causal xenophobia and racism and sexism that we see on an everyday basis. Those are the things we can adopt and embody and that definitely prolonged the period in which I was denying myself... these... denying myself even the capacity to answer these questions.

(Clark, 22, Transmasculine, Chinese, 1.5 Generation)

I've kind of thought of it to myself as digging out an abscess or something, it really kind of sucks... because the work that I have to do to be able to work through all the internalized grossness instilled upon me, like, thinking of people who are not cis-heterosexual as less than, and even though I don't believe that, having to try to dig that out and recognize that, and be like, "That was a part of my experience, but I don't believe it," and what does that mean for how I see myself. There's still a lot of selfloathing and self-hatred I'm trying to let go of, and. It's difficult! Because there's not a switch that you can be, like, "Oh! I accept this part of me now!" [laughs] You know?

(John, 24, FTM Trans, Mixed, 2nd Generation)

Not only was being trans shameful, it also meant being trans will result in having 
a difficult life.

When I was young, there was a lot more...thinking like, you know, being gay was wrong or like being a dyke was bad or being trans meant that you were going to be ostracized forever and fit nowhere or that if you're queer, you're going to have a hard life.

(Mik, 42, Nonbinary, Chinese, 1.5 Generation)

A couple of participants identified internalized messages that they received from

the trans community and the mainstream trans narrative. For example, due to the

internalized messages that being trans is hard and identifying as nonbinary, Mik (42,

Nonbinary, Chinese, 1.5 Generation) felt they were not trans enough because they didn't

struggle the same way they had seen other binary trans people.

And before, I think I was afraid. Like, I didn't earn the right to be transmasculine yet, because I hadn't gone through surgery or the pain.

And so, I think I was....I didn't want to....I didn't want to I think lessen the significance of our trans brothers and sisters who have really struggled so much more in finding their own trans identity, in terms of like, where they're doing injections at a young age or going through surgery or like, you know, there was a lot more....I think there's a lot more hardship for our trans community for those who really sought to transition.

(Mik, 42, Nonbinary, Chinese, 1.5 Generation)

\section{Theme: Coming Out}

While participants had reasons to be hesitant in their coming out process, they also had reasons why they chose or were able to come out. This theme described in detail reasons participants decided to come to themselves and others, regarding their gender identity. Four higher order categories manifested within this theme (see Table 8): Trapped; Distance as Protection; Protective Factors; and Authentic Self, Better for Family.

Trapped. Some participants described feeling trapped, whether it was in their environment/surrounding or in the distress they were experiencing in their own bodies. They felt trapped to the point where the only way out was to come out. For Jaymes (21, 
Male, Korean American, 2nd Generation), he felt trapped in his uncertainty about himself

\section{Table 8}

Coming Out theme and higher order categories.

\begin{tabular}{ll}
\hline Theme & Higher Order Categories \\
\hline \multirow{3}{*}{ Coming Out } & Trapped \\
\cline { 2 - 2 } & Distance as Protection \\
\cline { 2 - 2 } & Protective Factors \\
\cline { 2 - 2 } & Authentic Self, Better for Family \\
\hline
\end{tabular}

and his gender identity, where the only way for him to better understand himself was to

take those initial steps in exploration:

I wasn't even sure at the time, like, "Oh, this fits me" or whatever. Like, I had spent a while thinking, "Does this label fit me? Does it speak... Is it me?" And after a while I just sort of, like, "Well, I'm not really going to know if nothing keeps happening." Like, if I'm still in this questioning stage or this sort of.... Like, I'm going to be in this questioning stage forever if I don't actually try some of the things that I want to do. Like, be seen as a guy in some way. Like, to get guy's clothes or a binder or whatever. This won't happen unless I come out to my parents as something that they can kind of get, even if I don't really get it at the time...And [I] was just kind of sick of being uncertain. Or just being....very certain that I'm uncertain. And being like, "I need to do something. I need to try some of these things." Like, a change needs to happen, basically. And that feeling of, like, a change needs to happen, had been kind of cooking for a while.

(Jaymes, 21, Male, Korean American, 2nd Generation)

Clark (22, Transmasculine, Chinese, 1.5 Generation) described feeling trapped in

hiding himself from his parents when he was home, to the point where it was causing him distress:

For me it came down to would I be able to live with myself if I continued to present as a woman for another 4 years until they [parents] thought it was okay? You know. At what point am I still waiting for their approval to stop? At what point does that stop? And I think I came to this point about three months into my transition where I felt like okay I'm going to keep doing this, I can't just keep compromising with my parents they will take a while, you know what I mean? 
(Clark, 22, Transmasculine, Chinese, 1.5 Generation)

Even though he wanted to wait for his family, the pain of not taking steps towards transitioning was evident for Carlos (30, Trans man, Filipino, 2nd Generation) as he was surrounded by other trans people who were able to proceed in their own processes. Feeling left behind and experiencing dysphoria was difficult for him.

It got to the point where I was kind of dying from it. I really didn't, I wasn't doing well at all, and I, like, had been part of the trans community or the past two years, and seeing my peers get on $\mathrm{T}$ and progress and all this stuff.

(Carlos, 30, Trans man, Filipino, 2nd Generation)

Distance as Protection. Having physical distance between participants and their families (including extended families) and API communities allowed them the space and freedom to explore their identities, come out to themselves, and socially/medically transition. These participants expressed more confidence in their process, such that they were able to meet and be a part of communities as well as not feel beholden to their families and family values. They weren't afraid of being observed by their families and API communities.

Because I do go to school in (another state), there's a little bit more separation there for me to be a little bit more free...A lot of it had to do with the fact that I wasn't around my parents at the time of this boost of confidence. So I felt a little bit of freedom. (Clark, 22, Transmasculine, Chinese, 1.5 Generation)

Protective Factors. Several participants named protective factors in place that allowed them to feel more empowered and confident to come out. Unlike the other higher order categories in this theme, these protective factors were stable factors throughout their identity processes. The stability and protectiveness in these factors allowed participants more consistent space to navigate their environment and negotiate 
their identities and values. Having never seen familial rejection occurring in her

community and knowing that her family will be supportive gave Priya (41, Trans woman, Indian American, 2nd Generation) a sense of resiliency:

The way that Indian families function traditionally, the fact that nobody gets divorced, the fact that, you know, like, this idea that your parents would kick you out and you'd wait at the curb to call an Uber or taxi by telephone, is driven by movies and maybe my experience of European Americans. It's not something that I had ever seen first-hand with my community. And so, you know, I kind of already knew that that wasn't going to happen. That people would stand by me. So it's given me like not only a protective factor, but an internal sense of resiliency and an internal sense of when things didn't go perfectly, I knew that they would be okay.

(Priya, 41, Trans woman, Indian American, 2nd Generation)

Other identities and statuses also provided participants with some reassurance in

their processes. Priya (41, Trans woman, Indian American, 2nd Generation) identified

her educational and socioeconomic statuses as protective factors that gave her a sense

that things will be okay if she came out:

My parents both have master's degrees. And so, with that, that set me up. Like, I always knew that I would go to grad school before I even knew what grad school was. And then I went to grad school twice. Once in engineering and once in psychology. And so, I was really cognizant of the fact that I was set up in a place where I had a lot of education, specialized skills, a lot of income. And all of these things in the long run meant things would be okay, even if it was uncomfortable...Um, and so, I think, like, all of this acted as a buffer and a protective factor.

(Priya, 41, Trans woman, Indian American, 2nd Generation)

One's place of residence appeared to be impactful for participants. Living in a

liberal city and aware of the culture of acceptance there gave John (24, FTM Trans,

Mixed, 2nd Generation) the strength and assurance to come out:

I think that's largely a function of where I live [laughs]. I live in a very liberal city, I live in _ _ so it was kind of a given that when I would come out to my friends, they would be okay with it, you know. That was something I could kind of count on, whereas say if I lived in Kansas, or 
anywhere else, I would have to prepare myself to be like, "Oh, I'm gonna lose relationships over this." There're gonna be people that will no longer want to contact me or have anything to do with me because of something that is a part of myself that I can't help! You know. So kind of having that just positive vibe from the people and the culture here definitely strengthens me. I'm not sure I'd be able to take this so bluntly and head-on had I lived somewhere else.

(John, 24, FTM Trans, Mixed, 2nd Generation)

Authentic to Self, Better for Family. Being hidden and/or emotionally distant from their family created a barrier in the closeness participants were able to experience with their family. Some participants expressed feeling like they were lying to their families. Others didn't feel like they would be able to be in the same environment with their families. All of these situations not only prevented participants from being authentic to themselves and their identities, they were also going against their families' values. Ultimately, being authentic to themselves allowed these participants to be better and more fully engaged members of their families.

I think one of the things I've come to know for myself, or like, tell myself so that I can move forward, is like, it is most important that I am okay. Because I can't be a part of my family if I'm not feeling whole in who I am and feeling full in the whole. So, therefore, my decision to medically transition and those kinds of things, has to align with, um...is also part of the family comes first thing.

(Z, 25, Genderqueer, South Asian, 2nd Generation)

\section{Theme: Personal Values}

While participants were influenced and socialized with cultural values from multiple sides, what they decided to keep as their own personal values was their choice and influenced their worldviews. At the end of the day, individuals ultimately picked and chose which values were most important and authentic to themselves. The higher order categories under this theme represented the values that were kept and embodied by the participants, which were not always consistent with the values of their cultures of origin 
and mainstream American society. The higher order categories were (see Table 9):

Family and Community Importance; Filial Piety; Saving Face; Hard Worker; Social

Justice; and Live Life to the Fullest.

\section{Table 9}

Personal Values theme and higher order categories.

\begin{tabular}{lc}
\hline Theme & Higher Order Categories \\
\cline { 2 - 2 } Personal Values & Family and Community Importance \\
\cline { 2 - 2 } & Hierarchal Deference \\
\cline { 2 - 2 } & Preserving Honor \\
\cline { 2 - 2 } & Hard Worker \\
\cline { 2 - 2 } & Social Justice \\
\hline
\end{tabular}

Family and Community Importance. Even as an adult, participants continued to believe that family and community were just as important as ever. Even living in an individualistic society for the majority, if not the entirety, of their lives, participants continued to identify family and community as priorities in their lives.

Uh, I mean, that family valuing family is a very important aspect that I continue to find important... But also, I guess like, still managing to respect family anyway. So just... and also...yeah, so like, also maybe like some ideas of providing for family is an important value and I think in some ways that shapes my gender presentation. At least my gender expression and my gender role in family, in some ways. (RS, 34, Transmasculine queer, Taiwanese Chinese, 2nd Generation)

For Mik (42, Nonbinary, Chinese, 1.5 Generation), their personal value of family and community was not associated with any cultural values. Rather, they described their personal value was theirs alone.

Like, this Asian flytrap of, like, you have to stick to these beliefs of being, like, you have to all stay together even if you all hate each other and you all stab each other's backs and talk shit, but you're still going to be there for one another. And I hate that, because I'm like, it's not authentic. Just because they're related to you does not mean you have to go to their house 
and have dinner with them just because they're family, because you fucking hate each other. Like, I don't understand. I just...there's so many things about Asian culture that I...it drives me nuts. And I really want to have zero part of it, but of course there's still other parts of me that are very ingrained, such as wanting to take care of my mom and my family. But I don't look at that as being Asian or being Chinese. I look at that as just being a good human being.

(Mik, 42, Nonbinary, Chinese, 1.5 Generation)

Hierarchal Deference. The API cultural value of filial piety, such as respecting the social hierarchy and showing deference to elders, continued to be a central aspect of some participants' lives despite their acculturation into mainstream American society. It appeared that this value was so ingrained and internalized that they have incorporated it into their own set of personal values. This higher order category differed from that of a similar prior category of Filial Piety, such that the present category pertained to values that are currently held rather than values that were attempted to be socialized by family and community during childhood.

I still have the idea of, really strongly ingrained in me, very traditional Asian values of, like, respecting elders, showing deference. (John, 24, FTM Trans, Mixed, 2nd Generation)

Preserving Honor. This higher order category was consistent to the value of saving face, which attempted at not only preserving the honor of oneself but also the honor of those around them. Only one participant mentioned continuing to attempt to preserve honor in his everyday life, speaking to the degree in which this value, and similar others, were ingrained, internalized, and maintained. While only one participant made up this higher order category, it would be remiss to ignore that this was a current salient personal value. It was important to notice that it was not always related to the participant's identity process. 
So, I actually realized this a couple of days ago, where... I was trying to figure out, there is a disconnect in the conversation I was having between a researcher who was trying to draw my blood, and myself. And he kept profusely apologizing, I'm a really hard stick, so he had to stick me twice. And for me, I expect this, 'cause like, I just know it's gonna happen. And he would just, he could not get over the fact he had to stick me twice, and I kept trying to be like, "Oh, don't worry about it," kind of like, "Please, let it go, it's okay, like, you're human." [laughs] I don't know, I was trying to like, give him a way out. And he just wouldn't accept that? And it made me feel very uncomfortable and I realized later, it was like, I just wanted him to be able to be like, "Oh, she's giving me an opportunity out, it's okay," kind of thing, and not being able to do that, not recognizing that I was trying to do that for him made me uncomfortable, too, if that made sense? It felt like I was trying to, like, "Hey, like, I'm giving you a way out to still have your dignity, to like, save your face, and you're not taking it, you're like, violating the social norm, what are you doing?" ...So he was seeing it as a personal failure, whereas I'm trying to be like, "Just because you failed - just, just take it. Take the way out, friend. Please!" ...To make us all feel more comfortable here. (John, 24, FTM Trans, Mixed, 2nd Generation)

Hard Worker. Some participants continued to identify with a work ethic that pushed them to work hard and strive for excellence, often to the extent of being hard worker was a part of who they were rather than a mere expectation. However, it was not due to cultural expectations anymore, but rather, because they truly wanted and believed it for themselves as part of their own personal value.

It was a lot of pressure. I think being Chinese means that you're always under a lot of pressure to do very well. And I think that is part of our culture, the way that we were raised. Very high expectations... Coincidentally, I think it's so funny, because I think now it's ingrained in myself. Like, no one else is putting this pressure on me anymore. But now, it's just so ingrained that I do it for myself constantly... I feel like for me, every step of the way, no matter how many obstacles, no matter how hard my life was, I always strived for excellence. For the best. And I felt like I have done more in my 42 years of existence than some people have done in a lifetime.

(Mik, 42, Nonbinary, Chinese, 1.5 Generation)

Social Justice. The idea of activism and social justice was a personal value/higher order category that emerged in the data and was outside the realm of cultural 
values previously mentioned. Some participants noted a personal value regarding advocating for social justice and equality for all as something they strived for in their lives. In its own way, it may have been a combination of both API and mainstream American cultural values (as well as experiences of discrimination and isolation) that facilitated the development of this personal value.

I'm also currently right now, I'm not, I try to be as part of the, as much as I can, a part of the social justice movement, but I'm also, I feel like it's overwhelming. It's not my forte. But I know it's important, so I want to keep doing it, or find people who are into it and you tell me what to do. Tell me what's going on and I'll support that, type of thing... And so, I remember growing up watching myself "Terminator 2," you got to fucking fight for the little guy and like, protect, protect, protect. And I guess, because I also, for myself, growing up so fucking big compared to other Chinese kids, or other kids in general, like, anytime I picked up on the little guy, my brother was tiny and skinny as hell, so if I picked on him, I'm always the one in trouble, you know. I'm always the one at fault. It doesn't matter if he's the one who started it or whatever. So, like, that kind of value of, because you're bigger you have more power and you have the potential of hurting someone. You got to, you know, you got to not do that...I'm a little more vocal about things that I think are wrong. (Alex, 33, Genderqueer, Chinese American, 2nd Generation)

Living Life to the Fullest. Another value/higher order code that did not emerge in any prior value-based themes was the value of striving to live life to the fullest, regardless. While only one participant specifically endorsed this value, it was important to acknowledge the significance of how it can be used in navigating spaces and negotiating identities.

I think there was one thing about me that my mom pointed out...maybe like two years ago, was that she was like, she said to me, she was like, "Like, you really have no fear." I think she started off with, you know, with my surgery. I think it was my tattoos then with my surgery, and she was like, "You know, you're really brave. Like, you're not afraid." And then, I think it went further into her being basically acknowledging, like, she perceives me as someone who just lives without fear. And I think it made me think a lot about that. Like, I reflected a lot on that comment and I realized that I'm really glad that she saw that, because that is actually 
how I live. I live or I embrace living without fear. I embrace living without fearing, I guess maybe is better. Sure, I get scared about things and get nervous, but to truly live without fear, like basically, yes, I feel an obligation to take care of my mom but I also live without fearing that I have to fulfill or live based on their expectations.

(Mik, 42, Nonbinary, Chinese, 1.5 Generation)

\section{Theme: Integration}

This theme described the experiences of trans API participants of how they integrate and negotiate both their trans and API identities, if at all. The narratives documented in the data varied across the spectrum and degree of integration and negotiation, indicating a diversity of lived experiences and challenging any assumed single-dimensional, hegemonic identity process. Eight higher order categories illustrated the multiplicity of narratives (see Table 10): None Necessary; Ongoing Process; Struggle; Taking Care of Family; Centering the Self \& Showing Up; Meaning Making; Negotiation; and Authenticity.

\section{Table 10}

Integration theme and higher order categories.

\begin{tabular}{ll}
\hline Theme & Higher Order Categories \\
\hline \multirow{4}{*}{ Integration } & None Necessary \\
\cline { 2 - 2 } & Ongoing Process \\
\cline { 2 - 2 } & Struggle \\
\cline { 2 - 2 } & Taking Care of Family \\
\cline { 2 - 2 } & Centering the Self \& Showing Up \\
\cline { 2 - 2 } Meaning Making \\
\hline Negotiation \\
\hline
\end{tabular}

None Necessary. A few participants stated that their identities did not require any negotiation or integration process. These participants explained that their gender identity process was not related to any cultural factors or values, that their cultural values 
did not influence how they understood themselves, or that there were no conflicts between their cultural identities at all. For Mik (42, Nonbinary, Chinese, 1.5

Generation), they did not think their Chinese identity and culture influenced their gender identity in any way, such that their cultural identities were not related to each other:

Like, I don't even see myself really operating in the sense of being, like, "Oh, I'm Chinese so therefore this." Or, "I'm trans so therefore this." Like, I really just, really like, "I'm human, therefore this." And that's how I look at everybody else.

(Mik, 42, Nonbinary, Chinese, 1.5 Generation)

For Jaymes (21, Male, Korean American, 2nd Generation), how he understood himself in his respective identities appeared to be independent of the cultural values that were assumed to be attached to those identities:

I don't think they did, because I didn't think of them as Korean values. Uh, yeah, and the fact that I never had a very strong Korean identity to begin with means that I just never thought of it as a, "This is affected by it in this and this way." Just because it wasn't...Um, so other times my values have come into conflict. Um. Hmm. I can't really think of any. I'm sure there has been, but I just can't think of any. (Jaymes, 21, Male, Korean American, 2nd Generation)

Because no conflict was identified between his cultural values as they related to his identity process, RS (34, Transmasculine queer, Taiwanese Chinese, 2nd Generation) simply referred to his own personal values to make sense of his own identities, respectively and intersectionally:

I go with my gut, I guess. I mean, I feel like my gut just tells me what's right and I feel like there's some kind of basis... And I don't know how to explain that but like, just doing what's right because you feel that it's right and you know that it's right, as opposed to being swayed by any other argument, I guess. And so, I don't know if I always have a conflict between cultural stuff as opposed to doing that. (RS, 34, Transmasculine queer, Taiwanese Chinese, 2nd Generation)

Ongoing Process. For some participants, the identity negotiation and integration 
processes continued to be an ongoing process to this day. While they may have reached a

place where they felt more comfortable with their identities and in themselves, the

identity and value synthetization process continued to be present. This continuing

development was true even for participants who have been in their identity and

transitional process for years.

It took a lot of therapy, a lot of writing, a lot of supportive friends. But those messages never go away, they're just stuff you learn to live with, so I just feel like it... You know, it might not be the most, the tension, the conflict that is most salient at the time, but it's still going on, it's still something I negotiate every day.

(Carlos (30, Trans man, Filipino, 2nd Generation))

But I also didn't really identify, I also didn't want to be uh... I still saw a lot of shame in being openly Chinese. I still saw a lot of shame in embodying a lot of these characteristics. So, I'm still trying to figure out how exactly my trans-identity falls in relation to race, like how these two are related...W Was I going to become less Chinese by coming out as a trans person? Was I going to become more American by being a trans-person? Um, I don't know, I honestly don't. It's hard for me to, to think about culture and to think about also being American because my, I feel like my identity is always being negotiated. And it's hard for me to tell where this is coming from.

(Clark, 22, Transmasculine, Chinese, 1.5 Generation)

So, I actually don't know if I've completely reconciled it for the longest time, because I've only changed my name legally after 12 years of being trans or exploring this identity. I feel like I've been, I've been sort of milling around in this presentation for a long time, not doing much else, because I couldn't quite figure...couldn't quite figure out what I actually identify as.

(RS, 34, Transmasculine queer, Taiwanese Chinese, 2nd Generation)

Struggle. Most participants described the negotiation and integration process as a struggle for them. For Carlos (30, Trans man, Filipino, 2nd Generation), it was a process of "trial and error." For Z (25, Genderqueer, South Asian, 2nd Generation), it was complicated.

I grew up in a ton of really White spaces, so looking down at my body and 
be like, "Oh, it must just be because I'm brown. That's why I feel weird." You know, and later learning that, "Oh, and that gender stuff was there, too." And knowing that, sort of that weirdness that I felt was really, really complicated for me. Like, it's not just gender. It's not just queerness. It's not just brownness. It's all of it, all the time. (Z, 25, Genderqueer, South Asian, 2nd Generation)

This struggle was present not only between their trans and API identities, but also between their gender identities and expression due to cultural expectations as well as their ethnic identities and colonialism.

I think what I worry about is that... while I identify as trans, I also don't really identify as super-super-masculine. (John, 24, FTM Trans, Mixed, 2nd Generation)

I mean, Filipinos have a very complicated relationship with America and with a lot of countries, and - not a lot, but you know, more than just America. I don't know, I think that being Filipino in America is, you know, people talk a lot about east Asians and about model minority stuff, but when it comes to being a brown Asian, it is like, there is under - like, sure, I do get grouped with White people sometimes, but there is, under no circumstances that I am mistaken for White. I could never pass for White or anything like that. So, it means having a complicated relationship to colonialism and to capitalism, and some of that is about being queer too, but being Filipino in America is strange because, Filipinos are, I believe, the second largest Asian ethnic group in the U.S... I mean, it was a constant - constant dissonance. There was never, there was always friction there.

(Carlos, 30, Trans man, Filipino, 2nd Generation)

Taking Care of Family. Most participants were able to make sense of and come to terms with their identities and identity processes by continuing to take care of their families throughout the coming-out and transitional processes. In many ways, they were putting family first. Alex (33, Genderqueer, Chinese American, 2nd Generation) explained, "They haven't really shame me or anything like that. Or push me away for my identity. And so, I try to live that within my own self. And so, [I] try to give back that way." Similarly, Carlos (30, Trans man, Filipino, 2nd Generation) shared, "I was too 
invested in her process, of trying to be kind to her, and be accommodating, and to be -

because, you know, that's, like, part of the dynamic that was already set up, to prioritize

other people's needs ahead of mine."

Participants described reassuring their families by including them in the transitional decision-making process.

So I brought her to ___ with my sister, and I had this appointment with my doctor where I was kind of like, "Will you tell my family that I'm not going to die from this? You know, or like, be - you're a doctor in a place where people receive care."...But I had my folks sit down with the doctor and we talked about what the outcomes could be, and what, whether there's any long-term data, and all this stuff, which, you know, there was even less at the time. But, just enough to impress on my mom that it's, he's not going to die immediately, he's not going to take his first shot and then have a heart attack. But me and my family went out to lunch after that, and I could tell that my mom was just so inside her head, of just worrying, and she was fairly quiet, and the only question she had for the doctor was like, "How soon is this going to start happening?" I could tell that she felt like it was happening too fast even though I had been out for the past two years. And so, I definitely think that I put more emotional labor into my family's process than a lot of my White friends. That it was so important to me to have them along with me on this. Not just because I was living with my mom at the time, but just because, like, that's what you do, you know?

(Carlos, 30, Trans man, Filipino, 2nd Generation)

For Z (25, Genderqueer, South Asian, 2nd Generation), their decision to not

pursue various medical transition interventions at that time was in part due to feeling

protective of their parents' process:

And I'm trying to sort of take it one thing at a time to make it easier for them. I feel like it's a case-by-case thing, but also I don't want to bombard them with, "And then I'm going to do this. And then I'm going to do this. And then I'm going to do this." Even if that is how it might go, how it's probably going to go. And there are still things I don't know for sure yet. Like, I'm not certain I want to go on hormones. I'm pretty sure I will. But I haven't said that to my parents, because I don't want to scare them. And I also don't feel so, so certain that, like I do about the surgery, and I think that like... And I'm 25, like I've got plenty of time and space and they're supportive but I don't want to overburden them with things. 
So, the decision of putting my foot down is based on what they know and what they're ready to hear at the time.

(Z, 25, Genderqueer, South Asian, 2nd Generation)

Some participants also shared experiences of slowing down their process to match

their families' pace. Z (25, Genderqueer, South Asian, 2nd Generation) went on to

explain in detail their decision to meet their parents half-way by compromising on both of

their wants and needs, despite it causing some emotional distress:

I guess it was after I cut my hair, and when I cut my hair that was sort of like a huge turning point for me and my family, because both of my parents really, really didn't want me to do it. And I really wanted to. I made the decision to come home and do it with my mom there in the salon, which like I didn't have to do. I could've just come home with cut hair. I thought about doing that, but I was like, I think that would've break her heart more than going with her and saying, "This is what I want to do." And so, I told her I wanted to cut my hair and she was like, "No, you're not doing that." And I was like, you know, I was like 21 at the time. I was like, "Yeah, I can do this." And then, I...my mom took me to the salon and was like, "She wants to cut her hair!" And the woman who cut my hair was like, "That's great! I think it'll look great on you," whatever. My mom was like [sigh]. And I cut my hair and my mom was all distressed afterwards and she made me get my ears pierced right afterwards. It was terrible, because I was sitting in the um, I was sobbing hysterically because I really didn't want to get my ears pieced.

(Z, 25, Genderqueer, South Asian, 2nd Generation)

Some participants tried to understand their families' own emotional and lived

experiences. This often meant participants attempted to be patient as their families

navigated and negotiated their own transitional process.

So, the most salient example I can give is my mother won't recommend me to see dentists or doctors because they know other people that my mother also knows. And this might affect their kind of social standing within those groups. I, I'm not trying to judge my parents at all. I understand where they're coming. (Clark, 22, Transmasculine, Chinese, 1.5 Generation)

For Mik (42, Nonbinary, Chinese, 1.5 Generation), their conviction for their family meant that they were committed to taking care of family, regardless of their 
identity and identity process:

I don't know if my identity has anything to do with it, but being the only child, basically all of the responsibility falls on me. So, I don't know if it would've mattered what my identity was. I would still feel the same. Yeah, because even if I was a lesbian and cis female, I still would feel like the same about wanting to take care of my mom and handling all her stuff. I would still do the same. I think the only thing that would be different is probably my approach to her... I feel an obligation to take care of my mom, but I also live without fearing that I have to fulfill or live based on their expectations. So, like, she, maybe in her family, she feels like, "Oh, I have a daughter." But, for me, it was like, it really didn't matter where I fall on the spectrum, on the gender spectrum. It's that I'm going to take care of your no matter what. So, it really doesn't matter to me what you see me as and how I identify. None of those things really matter at the end of the day. It's that regardless, I'm going to do this.

(Mik, 42, Nonbinary, Chinese, 1.5 Generation)

Centering the Self \& Showing Up. Even if there was a struggle in the

navigation process with one's family, participants continued to center themselves and "show up" authentically as themselves. Sometimes, this meant putting themselves first ahead of their families' needs at times.

It was strange for sure, it made every single event awkward, and it was hard, too, for things like weddings and funerals and birthdays and shit, and I'm like, I want to participate in these things, and not make it about me, but everybody's going to be talking about me, either way. No amount of damage control around that is gonna stop them gossiping, so I can either never participate in my family activities ever again, or just fucking do it, you know. I like, show up and be the person that I am and they'll just have to deal with it. (Carlos, 30, Trans man, Filipino, 2nd Generation)

Meaning Making. As part of the navigation and negotiation process, participants attempted to make meaning of their identities and experiences along the way. Consequently, the process of meaning making helped empower them in their identity processes. The meanings in which participants were able to make for themselves varied. For example, Jaymes (21, Male, Korean American, 2nd Generation) was able to make 
sense of his gender identity by defining it as a biological and puberty process that was

appropriate for his age at the time:

But that's also a function of not being 14 anymore. Like, I feel like coming out at that age kind of tied my transition a lot to just sort of typical, like, typical milestones and growing up as an adolescent, in general. Like, it felt easier to incorporate it into my life, because that's kind of at an age when you're supposed to be growing and changing anyway. Like, especially physically transitioning, I kind of just thought of it as like, "Oh, I'm a late bloomer." Like, it felt more like that. Although, it's also not $100 \%$ bad. It was very easy for me to try and subsume it into a, "This is just my life." And I don't need to think about...I don't need to consider how it, like, how transition is important in kind of a different way, because I didn't really want to deal with that. (Jaymes, 21, Male, Korean American, 2nd Generation)

For Carlos (30, Trans man, Filipino, 2nd Generation) and Z (25, Genderqueer,

South Asian, 2nd Generation), they were able to redefine their gender identities, and gender expressions in ways that felt authentic:

There was a period of time where I was like, "Yeah, sure, let me try to, like, bend this a little bit, let me try and femme this up and whatever." And there's still some elements of that, but I feel very secure in my identity as a man. And I realize that trying to be fey for the sake of being queer enough was also disingenuous. That it was - that I was like, overshooting. That just because masculinity is toxic and oppressive and terrible doesn't mean that I need to be that guy. That part of being trans is making these new masculinities, and feeling, I just, I feel so solidly, immutably male that most of my, a lot of my anxieties around being out in public and everything like that really diminished the more I ended up being treated like a guy in society so, I just feel like, even though queer people have so much baggage around men and around masculinity, I just, at the end of the day I had to be me.

(Carlos, 30, Trans man, Filipino, 2nd Generation)

So my White ex-partner, like, I had said something like, "Maybe after I go on T, I'll grow my hair out. Why not? It might be fun." I had nice long hair, but maybe it'll be different when it's not attached to femininity in the same way and when I get to feel differently in my body, I can have lushes hair again. And she was like, "I don't understand why you would do that." And she was like, "It was so important to you to cut it." And I was like, "But it's not just like I cut it and now that gets to be it forever just because it was so important." Hair is important, period, you know. Like, 
regardless of how it specifically is going to show up. And so it can be just as important for me to then grow it back out. I don't know if I would. I was just thinking, you know. Who knows how I'm going to feel about my body in like 2 years, even. Um, like I feel like everything is constantly changing, and that is the only thing I know to be the only constant fact, right. But I kind of was like playing with that idea and I think, because hair can be such an important signifier, like I like the idea of being an Indian guy with long hair. Like, that could be really cool.

(Z, 25, Genderqueer, South Asian, 2nd Generation)

Priya (41, Trans woman, Indian American, 2nd Generation) shared that making

meaning of her identity as a trans South Asian person allowed her to better understand

her other intersecting identities.

And so, what's been beautiful about...uh... what's been beautiful about understanding my identities as an Indian American is that it poised me...it put me in a better place to understand my identity as a woman. What's beautiful about understanding my identity as a woman is put me in a better place to understand my Indian-ness, too, and my American-ness. And so, I think that that's the beautiful thing about spending time and doing work understanding our identities is that we get an invite into all these other aspects of who we are, too.

(Priya, 41, Trans woman, Indian American, 2nd Generation)

Overall, the acknowledgement that trans API identities and expressions are

diverse and multifaceted was empowering.

The beautiful thing about trans-ness to me is that it looks so different for so many of us. And I think that I wish more trans people got that. And that my story is my story, and I don't expect anybody else to like, be like, "Oh yeah, I had the exact same thing!" If they do, that's great, and we can talk about it. If it's different for you, I respect that and I would never doubt that that you're not trans because you didn't experience what I experienced. I wish more trans people got that. And I think more and more people are really getting how different it can be, but yeah, I wish we had more love and respect for how trans-ness shows up.

(Z, 25, Genderqueer, South Asian, 2nd Generation)

Negotiation. While not all participants noted having a negotiation process with their trans and API identities, there were many participants who did. Participants who experienced an integration process, they described actively engaging in the process of 
negotiation between their identities and values to come to a compromise between their selves in order form a whole self. The diversity and vastness of the human experiences, again, showcased a spectrum of negotiation processes. Some participants attempted to come to a compromise between their identities and values. For RS (34, Transmasculine queer, Taiwanese Chinese, 2nd Generation), the ability to compromise came at the realization that he was more acculturated towards individualism:

I think that the reason why I feel more American than Chinese, Taiwanese-Chinese, is because, like being an individual means that I can express my individual self. So I feel like it was easy to reconcile being trans and the individual expression of myself. Gender and that, versus trying to compromise and let myself be persuaded how I should act socially with other people. So, I think that's partly why I see myself as more individualistic as opposed to Chinese-Taiwanese, like more integrated.

(RS, 34, Transmasculine queer, Taiwanese Chinese, 2nd Generation)

Others were able to negotiate between their identities and values by acknowledging their own self-worth and valuing themselves over any single value system.

In the beginning, my father was supportive of it so I felt like if I have, you know, some kind of support from my family I could do it. But then after, after my mother knew I was trans I pretty much experienced this like lessening of support from my father and from my mother. And that made me realize that I had to make this decision for myself and that it didn't really matter how much I couldn't keep thinking about how much I'm hurting other people how much, because for me the value of myself was worth more than this idea that I was irrevocably changing this relationship between myself and my parents.

(Clark, 22, Transmasculine, Chinese, 1.5 Generation)

Negotiation identities and navigating spaces were also a matter of life-or-death for some participants.

I think when it starts to feel, like when it feels like life-or-death. I don't say that to sound dramatic, but just to be honest. I think when it gets to the point where if I have to do another summer of binding, that's going to 
be incredibly painful and it triggers a lot of my mental health stuff and it gets me to the point where I don't want to be alive. Or like, I can't live in this body anymore. When I can remind myself of that, "Okay, I'm not okay the way things currently are. So, I need to make these changes." And that is so.....and when it gets to that level for me, that's when it becomes necessary for me to speak up.

(Z, 25, Genderqueer, South Asian, 2nd Generation)

Alex (33, Genderqueer, Chinese American, 2nd Generation) shared that

regardless of how they identified, negotiated their identities, and navigated their spaces,

they were who they have always been:

Um, and maybe this is more directed to my family and the family members, like, I'm so afraid of coming out to them, but you know, I'm still me. I haven't changed. I'm still annoying sometimes. Really loving and you know, goofball and all that stuff. And really, you know, being trans, not trans, taking $\mathrm{T}$, not taking $\mathrm{T}$, having top surgery, not having top surgery, I'm still the same person. Maybe that's- I feel more like myself and I'm comfortable with myself, which has nothing to do with anybody else...And try to just be as open and loving as possible because, you know, as a genderqueer person, that's the point of using that label is, who the fuck cares. Like, I'm a little bit of everything, and just trying to live life, I guess.

(Alex, 33, Genderqueer, Chinese American, 2nd Generation)

The synthetization of identities and values, believing that one can hold on to

both/all cultural identities and cultural values, was mentioned. How one synthetized their

cultural identities and values also varied. Kai (24, Genderqueer, Taiwanese American, 2nd Generation) described utilizing one set of cultural values to live out the values of the other culture:

I'm still trying to figure that out. And as a Taiwanese person, in terms of how I practice these values or how I hold these values I can see how being an individual and wanting to give back to the community, I've been able to do that. I've been able to, being an individual, work my way through higher education, kind of learn how to be respectable, in terms of this white standard of what is respectable and be stable enough on my own to give back to the community. (Kai, 24, Genderqueer, Taiwanese American, 2nd Generation) 
Mik (42, Nonbinary, Chinese, 1.5 Generation) realized that they did not have to pick-and-choose their cultural values or cultural identities. Rather, the intertwining of both was possible and was achieved for them.

I want to be there for my family, but also, I'm fulfilling my dream. And like, there's the individual. There's that family obligation. And those two values of, who you are as an identity, definitely had to battle each other out. And in the end... what I realized is, you can do both. Those two can intertwine. And you can be an individual and still fulfill the duties and the values of family and being Asian, being Chinese, while doing that...Sure, it makes life a little challenging, but it's doable. If you believe in yourself, you can do it.

(Mik, 42, Nonbinary, Chinese, 1.5 Generation)

Z (25, Genderqueer, South Asian, 2nd Generation) synthesized their cultural

identities and values by tweaking cultural traditions in a way that would still feel

authentic to all parts of their identities. They explained:

Especially knowing my parents, I think they're very willing to have a conversation about how to challenge those things, especially with me, because it's not like I'm altogether just dismissive of anything. My parents kind of assume when I came out. especially as trans, they were like, "Oh, you're not going to want to have an Indian wedding." And I'm like, "No, I do. If we can make it work, I'll do it." You know, like, I don't feel like, "Oh, I can't do that because it's so, you know, old fashioned and gendered." Like, I don't feel like that at all. I feel like it can be really beautiful and special, as long as the traditions don't have to feel super triggering as someone who has dealt with gender identity stuff. And I think there are definitely ways to work around the gendered stuff. (Z, 25, Genderqueer, South Asian, 2nd Generation)

A few participants' negotiation process consisted of self-definition and acknowledging their own journey in their identity processes. Their understanding of themselves and their intersectional identities was up to them and them alone.

I know that fitting in to traditional society, traditional gender roles is not a part of who I am. And being kind of a strong-headed individual, I'm not afraid of that. I mean, I am, 'cause it's scary! But at the same time I'm like, I could choose to hide this part of myself and be kind of like, muffled and almost tortured for the rest of my life because I'll always have to hide 
that part of me, or I can just forge on ahead, on a path not quite many people take, but it's my path. Just because other people doesn't agree [sic] with my life decisions doesn't make them any less right or wrong, it's just, my decisions, part of who I am.

(John, 24, FTM Trans, Mixed, 2nd Generation)

I think one of the main ways has been in this sort of like, "I see the vision of who it is that I want to be and I want to just get there." Or especially mainstream trans narratives, it's like, "I woke up and realized I was trans and then I realized...." Or like, "I realized I was trans since I was a baby. And so now I'm 21 and I'm going to go to the doctor and inject myself with hormones and schedule my top surgery tomorrow." It's incredible that people can do that and that people feel solid enough in their identities to get there, and I have had to remind myself that my journey is mine for a lot of reasons and not just because it's mine and I get to decide everything. But also because there's a lot of factors that I consider differently than other people...I know it's because I've had to go at my pace, so it feels right for me. And that is okay. You know, I didn't want to go on hormones before I'm ready. And I don't want to make these huge decisions and be super anxious about them, because that's how it would be for me if I made them faster. Like, at this point, I know what I want and I'm going to get what I want. You know, if that makes sense. So, I think this sort individualism stuff makes for mainstream trans narratives, like has been really hard to get out of my head. And I think, too, because I wasn't somebody that has actively known I was in the wrong body since I was 4 years old, like, for a long time, I had to train myself to be like, "Just because I didn't know doesn't mean I'm not trans." (Z, 25, Genderqueer, South Asian, 2nd Generation)

I mean, I think with nowadays, I feel like it's up to me to define that. Like, had I been asked 10 years ago, I was...I was struggling to sort of carve out an Indian sliver of who I am. And now, like, I moved kind of away from thinking about myself as a coconut, and sort of think of myself as "I am who I am." And I maybe experience a sense of Indian-ness in a different way than, at this moment, like I maybe don't eat Indian food that often and I don't listen to Indian music or watch Indian TV or speak Tamil that well. But, I am also more aware of the deeper sort of level in which my way of thinking is an Indian way of thinking. And an American way of thinking. And, um, and so, I feel like a stronger sense that whatever I do is Indian American, rather than having to be Indian American in some way. Sort of in same sense that I don't have anything to prove to anybody as a woman. Like, I'm just who I am. And being a woman isn't something I do, it's just who I am. Being an Indian American now finally is who I am as well, and not something that I do.

(Priya, 41, Trans woman, Indian American, 2nd Generation) 
Authenticity. Ultimately, for many participants, individuals negotiated their

identities to find and achieve a sense of authenticity. The power of feeling and being authentic in themselves allowed participants to express themselves and experience their lives fully, and with compassion.

I feel like...I feel like there is a sense in which, like....being thought of as an Indian man never felt right at all. And there's this sort of sense in which our intersectional identities aren't compartmentalized. Meaning that, because the man piece didn't work, the Indian piece also didn't work. I'm much more comfortable being an Indian woman. Like, it makes sense to me and in that kind of sense, like it makes me feel more, like I think I feel more connected and less of a sense of ambivalence.... That as I'm navigating all of this, I have finally reached a place where I have a sense of who I am on multiple levels. And I can rely on that and from various input, from Indian culture, from American culture, from other things, but I know who I am and I feel able in my ability to find the right answers. (Priya, 41, Trans woman, Indian American, 2nd Generation)

It has allowed me to come through as myself in a different way than I had before. And now, I'm more like, I get to be the person that I have been, but more, like, I feel more comfortable as myself and so we all sort of, you know, as we get older, we're all coming more into our own... I'm getting to a point now where I look back at my life and love all the different versions of myself that has shown up and get that each of those versions in their genderedness has come to mean who I am now... And getting to a point where I can still say, "I love myself and deserve to be here on this Earth." Which, I don't feel every day. There's, you know, even in the last month, I've had some really bad episodes of just like, "It's too hard to be this person in this world." And like, that is because of the constant negotiation of all of those things that you were just laying out. So, I think it certainly have an effect. It's not like negotiation that necessarily leads to depression, but I think for me, it's been a long time of putting a lot of effort into saying, "I'm okay. I'm okay. I'm okay. I'm okay." And then kind of over compensate for where I'm at. And then now I'm at this place where I get that that negotiation is hard and I'm trying to be more softer and more compassionate with myself about it. And part of that being compassionate with myself about it is to say, like, "I have depression and that's okay, and I've dealt with it for a long time." And perhaps I even had it before I even understood myself as queer, but coming to terms with my queerness and my trans-ness and all of that stuff, like, has allowed to name depression as a reality for myself and not feel like I did something wrong...Like, everything about myself is connected to those things, for the most part. I'm really grateful to have gotten to a point in my life 
where I can name those things and nothing feels painful or incongruent. (Z, 25, Genderqueer, South Asian, 2nd Generation)

Compassionate healing was a consequence of authenticity for Kai (24,

Genderqueer, Taiwanese American, 2nd Generation):

I think struggling and trying to recover from an eating disorder, when I was a teenager up until my early 20 s I was trying to find, was trying to find a home in my own body and coming out as queer allowed me to find love and allowed me to feel intimacy. Not necessarily romantically not necessarily sexual but that allowed me to coming into myself a little more. And when I came out as, or when I started identifying as trans, I think that allowed me to forgive myself for a lot of the self-destructive things I did to myself when I was younger and didn't understand that I should take care of myself rather than punish myself. 'Cause I think a lot of the, I think, I think growing up punishment was actually a huge deal. Like, being punished for something was like a big thing and it was only when I turned 18 and 19 when my parents were like we're not going to punish you anymore because you're an adult. But even then, it's kind of fucked up. It's like, someone else is going to do it. I don't know. Yeah, I think definitely once I started identifying as trans I was able to forgive myself and try to be more gentle with my body. (Kai, 24, Genderqueer, Taiwanese American, 2nd Generation)

They expressed comfort in not only the sense of being who they were but also in

the flexibility to continue to explore themselves wholly.

So, like, learning the terminology, I think, as it was coming out, I think because I was so involved with the community that I was kind of privy to the growth of the terminology as they were developing. So, I got to learn them pretty much right away, because I was part of so many different communities. And I've always been very involved with the trans community and, like, the lesbian community, because I felt very much a part of all of them. But I think that coming out as someone who is nonbinary at, what, like 40 at the time, it was very much like...like another coming out again. But it was cool, because I finally felt like, "Okay, this, I'm getting closer, finally closer to the part of me that is completely authentic and genuine."

(Mik, 42, Nonbinary, Chinese, 1.5 Generation) 


\section{CHAPTER V \\ DISCUSSION}

The literature on API experiences has been long-established and plentiful, including research on LGBQ API experiences and identities. On the other hand, the field of trans research continues to be in its infancy, with the majority of its scholarship produced in the last decade (Moradi et al., 2016). Even during this time, trans research has been able to grow and expand into examining trans people of color experiences and identities. However, trans API experiences continued to be underrepresented in both fields. This study is the first of its kind in that it examines the identity process and phenomena of trans API individuals. In total, eight themes were found related to the navigation and negotiation process for this population: Navigating Belongingness ( \& Lack Thereof), American Cultural Values, API Cultural Values, Cultural Influences on Gender Identity, Hesitancy, Coming Out, Personal Values, and Integration. These findings were especially important in complicating existing hegemonic trans narratives dominating the trans research, which have been predominantly of White trans experiences as well as those of non-API people of color trans people. The findings further expanded and enhanced the existing literature. This study illuminated the complex process of navigating spaces and negotiating values in the gender identity processes of trans API individuals.

This chapter summarizes the findings in three components consistent to 
Moustakas' (1994) transcendental phenomenology methodology: Composite Textural Description (the "what"); Composite Structural Description (the "how"); and TexturalStructural Synthesis (the "essence"). The chapter concludes with exploring the study's clinical implications, limitations, and future research considerations.

\section{Composite Textural Description: The "What"}

The Composite Textural Description explained the "what" of the phenomenon of navigating spaces and negotiating cultural values among the trans API participants. Results discovered a spectrum of experiences with isolation and resiliency. Some participants experienced conflict between their cultural identities as they attempt to navigate and negotiate, some participants reported not experiencing any conflicts, and others described that their process continues to be ongoing. This finding alone was important in revealing the complexity of the trans API experience and challenges assumptions of a unified, homogenous experience for this community, as frequently implied in the literature that groups API folks and trans people of color together (e.g., Bith-Melander et al., 2010; Nemoto et al., 2004; Singh, 2013; Singh \& McKleroy, 2011; Xavier et al., 2005). That being said, results found that all participants mentioned struggling with some form of isolation. This was consistent with the literature on bicultural individuals and experiences with cultural isolation (Benet-Martinez \& Haritatos, 2005; Rudmin, 2003; Vivero \& Jenkins, 1999).

Experiences of navigating and negotiating the gender identity process was isolating for participants. Literature on bicultural individuals have found experiences of acculturative stress, part of which was due to the pressures of determining which culture to identify with more (Rudmin, 2003; Vivero \& Jenkins, 1999). Historically, 
acculturative stress has referred to racial/ethnic identities. This study broadened the definition of acculturative stress to other different cultural identities. This process of not knowing which culture or community they belonged to was found in the narratives, such that participants often questioned their belongingness in the communities in which their identities are situated. They noted not feeling like they belonged in either the trans community as an API person or their API culture of origin due to being a trans person. One participant, Alex (33, Genderqueer, Chinese American, 2nd Generation), often questioned their belongingness in their communities and whether they belonged to these spaces; they knew from their personal experiences that the answer was, "No." This was especially true and further complicated for one participant who identified as mixed race. John (24, FTM Trans, Mixed, 2nd Generation) shared his experiences of not feeling like he belonged in neither the Korean or White communities, let alone the trans community. Vivero and Jenkins (1999) referred to this phenomenon as cultural homelessness.

Based on identity theory, social identity is formed based on identifying and having membership with social groups (Burke \& Tully, 1977; Stets \& Serpe, 2013; Stryker \& Burke, 2000; Ting-Toomey, 2005). Identity negotiation theory also posited that individuals seek mutual affirmation of their identities in interpersonal interactions and community (Stets \& Burke, 2000; Ting-Toomey, 2005). However, identity formation and negotiation for trans API individuals may be a difficult process due to not being able to have membership or receive affirmation of their identities from either cultural group. This questioning of belongingness exacerbated their experiential isolation and was perpetuated by a number of experiences.

Vivero and Jenkins (1999) referenced discrimination from both cultural (i.e., 
majority and minority) communities as part of the bicultural acculturative stress. Similar to the acculturative stress literature, Vivero and Jenkin's study was addressing bicultural identities from a racial/ethnic lens. However, participants in this study also described experiences of discrimination and microaggressions on multiple fronts. They shared instances of transphobia from their API family and communities of origin, as well as racism from their trans communities. John (24, FTM Trans, Mixed, 2nd Generation) additionally experienced racism from both the White (towards his Korean identity) and Korean (towards his White identity) communities as a mixed-race person. Additionally, nonbinary and genderqueer participants described discrimination and microaggressions from the trans community due to its binary-centric nature. Unsurprisingly, these instances of discrimination were not uncommon and were consistent with the literature on racism, transphobia, and binary-centrism in these respective communities (Erich et al., 2010; Grant et al. 2011; Hendricks \& Testa, 2012; James, Herman, Rankin, Keisling, Mottet, \& Anafi, 2016; Lombardi, 2009).

Contributing to the isolation was also the experience of questioning themselves, their identities, and their belongness as a product of comparing themselves to others around them and noting their differences. Some participants described comparing themselves to their cis API and White trans counterparts, particularly related to their experiences in navigating spaces and bodies. Social cognitive theory and social comparison theory both suggested that individuals often self-monitor and compare themselves to others who are in similar situations or who have similar cultural identities (Bandura, 1991; Decety \& Sommerville, 2003; Festinger, 1954). Additionally, interpersonal comparisons were also a part of their childhood, such that they were 
comparing themselves to their White peers. The sense of isolation appeared to be rooted in their experiences at a young age and can continue to be a life-long process. The lack of representation and resources for trans API individuals provided them with instances of inaccurate comparisons, which further intensified their sense of isolation.

The results overwhelmingly revealed a lack of representation and resources, such as role models, visual/social representations, and information of people who not only identified like them but also looked like them. This impacted the degree to which participants believed they belonged to these communities. This appeared to be consistent with social representation theory, which posited that exposure to social representations and communities influences the opportunities to engage and communicate information with those groups (Moscovici, 1984; Rateau, Moliner, Guimelli, \& Abric, 2011). There is a plethora of sources people can draw social representation from, such as families, culture, communities, and media (traditional and social). It was difficult for individuals to determine their own membership without such representation. Within this study, the lack of role models and visual representation created a sense of isolation in which they were unsure of their memberships to their cultural groups. Group membership was further complicated when they are simultaneously experiencing discrimination and microaggression towards an "outside" identity. Social representation theory and social identity theory both supported the belief that group membership and representation facilitates the communication and transferring of information within the group. Conversely, having a lack of such group membership can hinder the collection of information necessary for understanding oneself within the group context. Thus, it was of no surprise that the lack of family support, access to community, and overall 
information made it difficult for participants to process and make sense of their experiences. The lack of language/terminology and overall language barriers further made it difficult to communicate their experiences to their families and communities. Language barriers and difficulty with communication between generations, often leading to family conflicts, have been consistently found in the literature on immigrant families and intergenerational familial dynamics (Salant \& Lauderdale, 2003; Shin, 1994). These experiences that facilitated isolation were related to the cultural identities and values that participants held, making them apparent throughout the process.

Part of the navigation and negotiation process for trans API participants was the awareness and acknowledgement of their cultural identities and respective values. Participants shared experiences with cultural values that were consistent with the literature on individualistic (e.g., individualism, putting self before others, being an individual) and collectivist (e.g., family first, filial piety, saving face) values among American society and API family/community of origin, respectively. Both individualistic and collectivistic values impacted how participants understood and negotiated their trans and API identities, as well as how they navigated their environments. For example, the collectivistic values of putting family first before the self and saving face delayed the gender exploration and coming out process for many participants, such that they were concerned about embarking on the process and shaming/embarrassing their families. Some participants also described avoiding certain API spaces to not bring attention to themselves as a trans person or shame to their family. These values were consistent with the literature on collectivistic values, such that family is the central pillar of one's life and there is pressure to make personal sacrifices for the sake and face of the family (Kim, 
Atkinson, et al. 2001; Kim, Yang, et al. 2001; Triandis et al., 1990).

Also, consistent with the literature on the differences between behavioral and value-based acculturation (Kim et al., 1999; Szapocznik et al., 1978), these collectivistic values continued to be significant in their own personal values even though all participants identified as 1.5- and second-generation API individuals. Research has historically found 1.5-generation immigrants hold values closer to first-generation immigrants than second-generation individuals (Koh et al., 2009). This study's participants may have leaned more towards individualistic culture and values because of their need to better understand their gender identity. Acculturative behavior also changes at a faster rate than acculturative values, such that individuals may behave outwardly consistent with the mainstream culture while maintaining traditional values, such as centering family (e.g., Bacallao \& Smokowski, 2009; Kim \& Omizo, 2006; Ryder et al., 2000). Thus, it was possible that engaging in participants' gender identity process was more related to acculturative behavior, which may be more consistent with the mainstream American society (trans community), while holding traditional values. For example, social and physical transitioning may be considered acculturative behaviors, while simultaneously wanting to include family in the transition process and centering the family may reflect the slower rate of acculturative values. Both Carlos (30, Trans man, Filipino, 2nd Generation) and Z (25, Genderqueer, South Asian, 2nd Generation) explained their decisions to include their families in their medical transition process were due to their valuing and centering of the family. Individualism was described as a mechanism that allowed participants the physical, emotional, and psychological space to explore and come out as their gender identities. It gave them permission to focus on 
themselves, rather than solely on their families. Per Carlos (30, Trans man, Filipino, 2nd Generation), American culture gave him permission to finally live for himself. Participants seemed to utilize individualism as part of their identity process.

The power of representation and resources was evident. It was only when participants were able to experience social representation and have access to resources that they were able to feel empowered to begin exploring themselves and their identities. Participants were able to engage with trans API communities, as well as trans communities in general, to observe possibilities, explore experiences, exchange information, and determine the degree of their own membership. Representation and resources empowered participants to make decisions for themselves and to proceed in their identity process given the information they were able to obtain. Individuals often used the internet for social support and information during their transition process, which allowed them to feel empowered, connected, and a sense of self-discovery (e.g., Cavalcante, 2016; Ross \& Scholl, 2016). Cheng (2009) found that positive experiences, such as a sense of belongingness, increases identity integration. While Cheng's study was related to those who hold multiracial identities, it may hold true for the intersectionality of trans and racial identities as well. A sense of community and subsequent feelings of belongingness was found to be facilitative of trans men's positive emotional experiences (Budge et al., 2015). These positive experiences from the power of finding resources, representation, and belongingness could explain how trans API individuals are able to navigate and negotiate their identity process.

Protective factors were forms of resources that were stable and consistent throughout participants' lives. Some participants acknowledged the existence of 
protective factors provided additional safety nets for personal exploration, navigation of spaces, and negotiation of cultural identities. Some protective factors identified by participants included educational and socioeconomic status, familial/social support, and liberal geographical location, which appear to be consistent with the literature (Barr et al., 2016; Budge et al., 2013; Budge, Chin, \& Minero, 2017; Katz-Wise, Reisner, WhiteHughto, \& Budge, 2017; Moody, Fuks, Pelaez, \& Smith, 2015). In general, protective factors have been shown to positively impact psychological and emotional health, as well as develop resilience. For trans people, especially trans people of color, protective factors were crucial and critical in their survival and resilience (e.g., Singh, 2013; Singh \& McKleroy, 2011). The combination of resources, representation, and protective factors within an individualistic social context cultivated a space for participants to come into their authentic selves as trans API people.

The process of negotiation towards authenticity found in this study was further nuanced when examining participants' ability to make meaning out of their values and experiences. Making meaning out of their identities encompassed a spectrum of experiences, ranging from acknowledging their salient identification to individualism/American culture to putting their self-worth above either cultural value systems. For some participants like Carlos (30, Trans man, Filipino, 2nd Generation), the negotiation process meant life-or-death and necessitated survival. Moody and colleagues (2015) found several transition-related factors that helped decreased suicidal ideation among their trans participants, such as coming out as their authentic identity, having a hope for transitioning, and finally transitioning. The 2016 National Transgender Discrimination Survey (NTDS) found that $40 \%$ of trans respondents had attempted 
suicide at some point in their life time, which remained unchanged from their 2011 NTDS report of 41\% (Grant et al., 2011; James et al., 2016). Transitioning as survival can also mean protection from discrimination and violence (e.g., Begun \& Kattari, 2016; Levitt \& Ippolito, 2014).

Other participants were able to make meaning out of both individualistic and collectivistic cultural values in such a way that actually facilitates and enables each other. This challenged social identity theory such that individuals code-switch between identities based on their social environment and the salience of each identity in certain situations (e.g., Toomey-Ting, 2015). The process of making meaning out of their experiences, identities, and personal values (rather than the actual meaning being made) was the core of participants' negotiation process towards claiming self-authenticity.

\section{Composite Structural Description: The "How"}

The Composite Structural Description focused attention on the feelings and emotions of the phenomenon - the experiences of navigating and negotiating cultural identities. Similar to what trans API participants experienced, how they experienced their navigation and negotiation process was also spread across a spectrum. Participants expressed a range of emotions that encompassed throughout their process. As trans API participants were experiencing isolation, the overwhelming emotion that accompanied it was feeling of loneliness. The feeling of loneliness continued to be ever present as they experienced instances of discrimination and microaggressions, questioned their belongingness in the world, and internalized cultural messages about their identities. It was of no surprise that loneliness was elicited, which has been repeatedly found in the literature for both the trans (e.g., Grossman \& D’Augelli, 2006; Levitt \& Ippolito, 2014) 
and API (e.g., Salant \& Lauderdale, 2003; Shin, 1994) communities.

For participants whose cultural values and identities began to feel conflicted, particularly when they wanted to explore their identities and begin transitioning, some feelings of guilt also emerged. Differences in behavioral and value-based acculturation put stress on individuals' psychological and emotional experiences, such that guilt may be experienced when an individual behaves in a way that appears incongruent with one's cultural values (e.g., Bedford \& Hwang, 2003; Liem, Lim, \& Liem, 2000). In general, acculturative stress have been found to be positively related to depressive symptoms (Romero et al., 2007). For a couple of participants, they felt uncertain of how to navigate the feelings of guilt and negotiate their identities/values without access to role models, representation, or resources to support them in that process. It further intensified feelings of loneliness, as well as the belief that they had to embark on their process on their own.

On the other side of the spectrum, participants expressed feelings of relief, curiosity, and even shock when they first discovered resources and social representation. Budge \& colleagues (2015) found that trans men's positive emotional experiences related to finding community. Barr et al. (2016) found that community belongingness is an important and positive component of trans individuals' identity process. There was a sense of community belongingness, rather than loneliness, when participants found other trans people, regardless of race and ethnicity. Though, in the current study, connecting with other trans API people provided participants with a sense of being completely understood. For participants, who acknowledged their access to protective factors, expressed a sense of security and safety. The literature acknowledged the importance of protective factors in trans individuals' ability to feel safe in their environment and secure 
in their relationships as they navigate their surroundings, experience their identity process, and build resilience (McGuire, Anderson, \& Toomey, 2010; Singh, 2013; Singh \& McKleroy, 2011).

Ultimately, participants expressed an overall sense of comfort and wholeness when they were able to be authentic in both of their cultural identities. Goldman and Kernis (2002) have found that authenticity positively relates to self-esteem and life satisfaction, and negatively associated with negative affect. Minority stress model may suggest that this positive impact could be in part due to less stress from not having to be hypervigilant and hide oneself and one's identities (Hendricks \& Testa, 2012; Meyer, 2003). It is possible that participants' sense of authenticity also provided them with a sense of overall positive psychological well-being in addition to feeling comfort and wholeness.

\section{Textural-Structural Synthesis: The Essence}

The essence, or the textural-structural synthesis, integrated both the composite textural and composite structural descriptions to deliver a synthesized description of the phenomenological experience. In this section, the composite descriptions were used to form a complex, yet whole, narrative of the navigation and negotiation process for trans API participants. The phenomenological experience of navigating space and negotiation one's complex identities did not start at the questioning or exploration of gender identities. Rather, it transcended gender. The navigation and negotiation of all their identities was the byproduct of their existence in the U.S.

Participants initially felt a sense of isolation, loneliness, and othering beginning in childhood. Witnessing negative external perceptions and behaviors (e.g., discrimination 
and microaggressions) directed towards them made them question their belongingness in the spaces they were occupying or were attempting to occupy. Participants questioned who they were and where they belonged, which was a lonely experience. Without resources or representation in their life, navigating these spaces was confined within the boundaries of what was familiar, though not necessarily congruent or authentic. As participants were becoming more aware of their gender identities, the lack of resources and representation further made it a lonely start to their processes where they grappled with their sense of self and often questioned if their experiences were real/valid. Some noted that they did not even have the language to describe their experiences, let alone describe it to their family where language barriers exist. The acculturation gap also created interpersonal and intergenerational conflict due to different communication styles and needs, which is consistent with experiences of acculturation and acculturative stress in the literature (e.g., Lee \& Mjelde-Mossey, 2004; Mui \& Kang, 2006; Pyke, 2005; Sue $\&$ Sue, 2007; Xia et al., 2013). For some, the isolation and loneliness went hand-in-hand and led to the belief, feeling, and even necessity of having to embark on their identity processes on their own.

While not true for every participant, many described cultural values initially felt conflicting and made it difficult to make sense of their gender identity as API individuals. It felt like a struggle. With no resources or representation to refer to, the isolation and loneliness exacerbated these conflicting experiences and feelings. Part of their negotiation process was their hesitancy to explore their gender and preference in leaning on what was familiar to them, which was their API value of putting family first. This may be due to the Mere-Exposure Effect, which is the social psychological phenomenon 
that explains our preferences for the familiar; it posits that humans prefer things that are familiar because they are more readily understood, seemingly safer, and, consequently, quicker to process (e.g., Litt, Reich, Maymin, \& Shiv, 2011; Reber, Winkielman, \& Schwarz, 1998; Zajonc, 1968). However, familiarity may not always yield positive outcomes (Litt et al., 2011). For most participants, putting the family first continued to be a personal value. Going against, or wanting to go against this cultural value, of putting family first was difficult for many participants, sometimes creating feelings of guilt and shame that delayed their exploration and coming out process. The delay in initiating the identity process may be considered a form of personal sacrifice, which is consistent with the literature on collectivistic values. However, delaying the identity process and suppressing one's identity have been shown to be associated with depression, anxiety, and suicidality (e.g., Moody et al., 2015). The data showed a number of things helped facilitate and support participants in this process, such as finding and accessing resources and forms of representation. They provided the spark of curiosity, combined with being in an individualistic space, which gave them permission to begin their journey towards wholeness and authenticity. In a way, they gave participants references of different possible ways of being.

Participants, like RS (34, Transmasculine queer, Taiwanese Chinese, 2nd Generation), noted being further acculturated into American society and identifying with a sense of individualism while holding personal values that were more historically considered collectivistic. Engaging in behaviors more aligned to their individualistic environments and living out their family-oriented cultural values created some generational conflict within their family and a sense of internal guilt for some 
participants. Resources/social representation and protective factors, as well the environment which they are situated in, allotted for a space that provided a sense of possible freedom for authenticity. This may also include physical distance as space for freedom of exploration. In addition, Kim and Omizo (2005) found that adherence to individualistic cultural values was associated with greater cognitive flexibility and personal efficacy. Perhaps having more cognitive flexibility and self-efficacy also provided participants with the capability to negotiate their cultural identities and experiences. While it was an ongoing process for several participants, many were able to negotiate their cultural identities by making meaning out of their identities and integrated experiences.

Interestingly, individual participants making their own meaning out of their experiences could be considered a form of self-determination. The concept of selfdetermination, particularly within the counseling literature, has been defined by the idea of being able to define the self and one's own identity independent of external influences and norms (e.g., Collazo et al, 2013; Diamond et al., 2011; Israel, 2004, 2005). However, that definition was often viewed and understood through a Westernized lens and neglect to consider what cultural values may impact how one makes sense of self-determination. This study challenged Westernized notions of self-determination by expanding it to consider individuals' personal decision to embody (or not) cultural/social influences, norms, and values. Attempting to delicately balance personal and community/family needs may cause distress among collectivistic individuals. Chun, Moos, and Cronkite (2006) suggested that these individuals may engage in adaptive coping strategies that can be misinterpreted as individualistic in nature, such as self-reliance—or in the case of the 
current study, self-determination.

Some participants were able to make meaning out of their experiences by understanding their process as exclusively separate from one another, thus no negotiation process was necessary. Other participants were able to acknowledge that their values do not have to be either/or. Rather, they were able to make meaning out of their values that allowed them to be consistent with both, such as putting their family first in other ways. Some participants made meaning out of putting themselves first as a way of taking care of their family, which challenges traditionally Western conceptualization of selfdetermination. From a cultural context, Bandura (2002) argued that personal efficacy and self-directedness is not necessarily valued due to individualism, but rather, it is crucial for personal success and growth. Additionally, he posited that personal efficacy and selfdirectedness are not antithetical to community (or family); instead, they are just as important for individuals as group-directedness. This supported the current study's findings, such that determining meaning in one's own authenticity does not equate opposition to family values. Rather, it was just as equally important to both the families and the individuals, themselves.

Determining a way to negotiate their gender identities by making meaning of their cultural values and experiences facilitated a sense of comfort and authenticity. Consequently, the sense of comfort and authenticity helped built a sense of resilience and empowered participants to continue negotiating their identities and navigating cultural spaces in ways that feels genuine and authentic for them. Schwartz and colleagues (2012) would have argued further that regardless of which cultural identity/group individuals attach themselves to - any or all identities/groups - the attachment itself to 
said identity/group can promote positive psychological well-being. Participants were able to define authenticity for themselves, allowing them to be truer to themselves, their own personal values, and their families.

\section{Clinical Implications}

The goal of this study was to explore the lived experiences of trans API individuals and their process of navigating spaces and negotiation cultural identities, which has yet to be fully captured in the current literature. The findings made apparent the complicated and complex process of the identity formation for this population as it relates to culture, values, and spaces. Comprehensive understandings of cultural spaces including family and community, intersectionality of cultural values, acculturative influences, personal cultural values, and their various influences on one's external and internal experiences were central in working with this population. This understanding can assist clinicians and trans API individuals alike on the possible ways to embark on the gender identity process with intersectional cultural identities.

Clinical interventions should assist trans API individuals in navigating cultural spaces. In particular, clinicians should provide support in navigating experiences of discrimination/microaggressions that perpetuate feelings of loneliness and isolation. Based on the study's finding, it may be therapeutic for trans API clients to find resources and social representation, as well as develop communities of similar others. Communities of other trans API people are especially powerful in creating a sense of belongingness. Clinicians could assist trans API clients in finding and building connections and involvement with trans API-specific organizations. Processing emotional experiences related to being amongst and interacting with these communities 
can deepen their sense of belongingness. Having an understanding of how these external, interpersonal experiences can impact the identity process is vital. Clinicians should collaboratively engage with clients in discerning the degree to which these experiences are internalized and influencing their understanding of themselves, their identities, and their processes. For example, clinicians could help trans API clients contextualize their experiences of cultural values and the influences those values can have on how they understand their authentic selves. Similarly, clinicians could also support clients in their understanding of how experiences of discrimination may have impacted their sense of belongingness and how this lack of belongingness may influence their self-perceptions. Additionally, clinicians should support trans API clients in identifying and building protective factors, such as establishing systems of support. Depending on clients' needs, clinicians may explore with clients existing protective factors to continue to foster, as well as determine areas in which more support and protective factors are needed.

Furthermore, clinical interventions should focus on assisting trans API individuals in their own identity process by facilitating client-driven meaning-making processes of their cultural identities, values, and spaces. It is important for clinicians to recognize the complicated ways individuals may make meaning out of their identities and experiences, such that the choosing of family over some components of own's identity may not necessarily equate to personal sacrifices or a loss of self-autonomy that is harmful to the identity process. Rather, it may be clients' own way of making meaning of their values to best negotiate their cultural identities. Positive and open environments are crucial for trans API individuals to continually explore and make their own meaning of their identities, as they navigate their cultural spaces and negotiate the intersectionality of their 
cultural identities. Because a safe environment allows clients to take risks and be vulnerable, clinicians should be diligent in being affirming and validating of clients' identity processes and the meaning they are making out of their experiences. Collaboration throughout the therapeutic process can empower clients in their own personal growth. All clinicians make clinical biases and assumptions; however, cultural humility and being open to examining, challenging, and discarding these biases and assumptions to authentically engage with trans API clients is necessary and vital for the therapeutic alliance.

\section{Limitations and Future Research}

While the findings of the current study enlightened the process in which trans API individuals navigate spaces and negotiate cultural identities, there were several limitations. First, the study only collected a sample size of ten participants. While it is generally accepted as a modest sample size for a qualitative study, much like any study, its capability to generalize to the overall trans API population should be considered (Trotter, 2012).

The study recruited mostly individuals who were female assigned at birth and masculine-of-center. Only one participant identified as a trans woman. This limited the study's comprehensive understanding of API trans women's identity processes, as their experiences may differ from those who are trans masculine. In particular, API trans women encounter distinctive forms of othering and isolation beyond racism and general transphobia, such as discrimination and microaggressions related to misogyny and living in a patriarchal society. This is true for trans women of color, in general, such that they experience higher rates of discrimination, violence, and murder (e.g., Grant et al., 2011; 
Grossman, D'Augelli, Howell, et al., 2005; Grossman, D'Augelli, Salter, et al., 2005; James et al., 2015). API trans women representation in research would expand the literature even further. Future studies should examine the navigation and negotiation process of API trans women and feminine-of-center API trans individuals.

While the study attempted to recruit individuals across the API spectrum, the data was made up of entirely of Asian-identifying participants and no representation of individuals identifying as Pacific Islander (PI). Thus, based on the sample of the study, the study's use of API as the comprehensive nomenclature appears to be a misnomer. This limited the understanding of the full spectrum of the API community and continues to perpetuate the API literature that speaks for PI communities without extensive representation. Future studies should include, if not solely focus on, PI voices and lived experiences. PI-centered research, particularly related to PI individuals navigating Western spaces and negotiating individualistic values, is especially needed in the trans literature.

Additionally, all participants identified as 1.5 or second generation. An advantage of this sample was being able to get a glimpse of the impact of acculturation (behavior versus value) on the identity process of trans API individuals. However, it continued to limit the understanding of generational factors on the identity negotiation process beyond the second generation. The literature has shown vast differences between first and third generations (and later). Even amongst first and second generations, there are considerable differences (Koh et al., 2009). It was curious to see what and how the shifts in value and behavioral acculturation for later generations of trans API individuals. Future studies should investigate how later generational factors influence how one 
navigates spaces and make meaning of their experiences to negotiate their cultural identities and values. The impact of generational gaps on family dynamics and how family dynamics impact navigating cultural spaces/negotiating values should also be considered.

Other future research opportunities are vast and plentiful. Future research should consider cross comparison of different ethnic API cultures/values on navigation/negotiation experiences. Nuanced differences in socio-historical-political factors could contribute to the interpersonal and intrapersonal dynamics of the gender identity process. Examination of these possible differences would complicate the trans API narrative even further, as well as expand and enhance the diverse understanding of this population.

Much like nuanced differences in ethnic cultural experiences, future research should also examine how differences in geographical location may impact the identity process for trans API individuals. The impact of geography on cultural/family dynamics and access to resources/representation should also be assessed. While not formally reported in the results of this study, some variances in participants' experiences based on where they grew up in the U.S. was observed. For example, some participants noted growing up in an API enclave while others grew up in a pre-dominantly White area. Some participants shared varied degrees of access to resources and representation, as well as protective factors, based on their geographical location. A few participants even described how their families' established connection to API enclaves and communities impacted the ways in which they navigated those API spaces (i.e., distancing or withdrawing from API spaces, families, and events; building community in other API 
spaces).

It is further important to consider research related to the family of trans API individuals, as the family unit is the main transmission of cultural values and is a major component in the negotiation process. From a family systems perspective, future research should investigate family dynamics such as those that are hindering and facilitative of the identity process. Analysis of families' own emotional experiences throughout the identity process of their family member should also be considered. It may be valuable to examine how API families also navigate cultural spaces and negotiate their family's identities when they have a trans family member. Especially for clinicians working with families of trans API individuals, the clinical implications are numerous and potentially impactful.

Lastly, future research on trans API individuals and resilience is necessary. The power of resources (e.g., access to community and support), representation (e.g., role models), and protective factors (e.g., stable factors such as socioeconomic status) was present in the current study. Participants named these components as the catalyst of their negotiation process and/or the grounding element that provided a sense of comfort and safety during the process. However, little continues to be known how these components impacted trans API individual's resilience and mental well-being. Clinical implications resulting from these studies are multifold. Further research would be particularly important in building trans API clients' resilience during times of distress and environmental hostility. 


\section{REFERENCES}

Abraham, M. (1999). Sexual abuse in South Asian immigrant marriage. Violence Against Women, 5, 591-618.

American Counseling Association. (2010). American Counseling Association competencies for counseling with transgender clients. Journal of LGBT Issues in Counseling, 4, 135-159.

American Psychiatric Association. (1980). DSM-III: Diagnostic and statistical manual of mental disorders. American Psychiatric Association.

American Psychiatric Association. (1987). DSM-III-R: Diagnostic and statistical manual of mental disorders. American Psychiatric Association.

American Psychiatric Association. (1994). DSM-IV: Diagnostic and statistical manual of mental disorders. American Psychiatric Association.

American Psychiatric Association. (2000). DSM-IV-TR: Diagnostic and statistical manual of mental disorders. American Psychiatric Association.

American Psychiatric Association. (2013). DSM 5. American Psychiatric Association. American Psychological Association. (2015). Guidelines for psychological practice with transgender and gender nonconforming people. American Psychologist, 70, 832864.

Asakawa, K., \& Csikszentmihalyi, M. (2000). Feelings of connectedness and internalization of values in Asian American adolescents. Journal of Youth and 
Adolescence, 29, 121-145.

Bacallao, M. L., \& Smokowski, P. R. (2009). Entre dos mundos/between two worlds: Bicultural development in context. The Journal of Primary Prevention, 30, 421451.

Bandura, A. (1991). Social cognitive theory of self-regulation. Organizational Behavior and Human Decision Processes, 50, 248-287.

Bandura, A. (2002). Social cognitive theory in cultural context. Applied Psychology, 51, 269-290.

Bariola, E., Lyons, A., Leonard, W., Pitts, M., Badcock, P., \& Couch, M. (2015).

Demographic and psychosocial factors associated with psychological distress and resilience among transgender individuals. American journal of public health, 105, 2108-2116.

Barr, S. M., Budge, S. L., \& Adelson, J. L. (2016). Transgender community belongingness as a mediator between strength of transgender identity and wellbeing. Journal of Counseling Psychology, 63, 87-97.

Bedford, O., \& Hwang, K. K. (2003). Guilt and shame in Chinese culture: A crosscultural framework from the perspective of morality and identity. Journal for the Theory of Social Behaviour, 33, 127-144.

Beemyn, G. (2013). A presence in the past: a transgender historiography. Journal of Women's History, 25, 113-121.

Begun, S., \& Kattari, S. K. (2016). Conforming for survival: Associations between transgender visual conformity/passing and homelessness experiences. Journal of Gay \& Lesbian Social Services, 28, 54-66. 
Benet-Martínez, V., \& Haritatos, J. (2005). Bicultural identity integration (BII):

Components and psychosocial antecedents. Journal of Personality, 73, 10151050.

Berry, J. W. (1980). Acculturation as varieties of adaptation. In A. M. Padilla (Ed.), Acculturation: Theory, models, and some new findings (pp. 9-25). Boulder, CO: Westview.

Berry, J. W. (1997). Immigration, acculturation, and adaptation. Applied Psychology: An International Review, 46, 5-34.

Bess, J. A., \& Stabb, S. D. (2009). The experiences of transgendered persons in psychotherapy: Voices and recommendations. Journal of Mental Health Counseling, 31, 264.

binaohan, b. (2014). Decolonizing trans/gender 101. USA: biyuti publishing.

Bith-Melander, P., Sheoran, B., Sheth, L., Bermudez, C., Drone, J., Wood, W., \& Schroeder, K. (2010). Understanding sociocultural and psychological factors affecting transgender people of color in San Francisco. Journal of the Association of Nurses in AIDS Care, 21, 207-220.

Blackwood, E., \& Johnson, M. (2012). Queer Asian subjects: Transgressive sexualities and heteronormative meanings. Asian Studies Review, 36, 441-451.

Blumer, M. L., Green, M. S., Knowles, S. J., \& Williams, A. (2012). Shedding light on thirteen years of darkness: Content analysis of articles pertaining to transgender issues in marriage/couple and family therapy journals. Journal of Marital and Family Therapy, 38, 244-256.

Bockting, W. O., Miner, M. H., Swinburne Romine, R. E., Hamilton, A., \& Coleman, E. 
(2013). Stigma, mental health, and resilience in an online sample of the US transgender population. American Journal of Public Health, 103, 943-951.

Bockting, W., Robinson, B., Benner, A., \& Scheltema, K. (2004). Patient satisfaction with transgender health services. Journal of Sex \& Marital Therapy, 30, 277-294

Bowleg, L., Huang, J., Brooks, K., Black, A., \& Burkholder, G. (2003). Triple jeopardy and beyond: Multiple minority stress and resilience among Black lesbians. Journal of Lesbian Studies, 7, 87-108.

Broadus, K. W. \& Minter, S. P. (2014). Sex and gender development. In L. EricksonSchroth (Ed.) Trans Bodies, Trans Selves: A Resource for the Transgender Community, (pp. 174-211). New York City, New York: Oxford University Press.

Budge, S. L., Adelson, J. L., \& Howard, K. A. (2013). Anxiety and depression in transgender individuals: the roles of transition status, loss, social support, and coping. Journal of Consulting and Clinical Psychology, 81, 545.

Budge, S. L., Chin, M. Y., \& Minero, L. P. (2017). Trans individuals' facilitative coping: An analysis of internal and external processes. Journal of counseling psychology, 64, 12-25.

Budge, S. L., Orovecz, J. J., \& Thai, J. L. (2015). Trans men's positive emotions: The Interaction of gender identity and emotion labels. The Counseling Psychologist, 43, 404-434.

Budge, S. L., Rossman, H. K., \& Howard, K. A. (2014). Coping and psychological distress among genderqueer individuals: The moderating effect of social support. Journal of LGBT Issues in Counseling, 8, 95-117.

Burke, M. C. (2011). Resisting pathology: GID and the contested terrain of diagnosis in 
the transgender rights movement. Advances in Medical Sociology, 12, 183-201.

Burke, P. J., \& Tully, J. C. (1977). The measurement of role identity. Social forces, 55, 881-897.

Butler, E. A., Lee, T. L., \& Gross, J. J. (2007). Emotion regulation and culture: are the social consequences of emotion suppression culture-specific? Emotion, 7, 30-48.

Cahill, S., Battle, J., \& Meyer, D. (2003). Partnering, parenting, and policy: Family issues affecting Black lesbian, gay, bisexual, and transgender (LGBT) people. Race and Society, 6, 85-98.

Carrera, M. V., DePalma, R., \& Lameiras, M. (2012). Sex/gender identity: Moving beyond fixed and 'natural' categories. Sexualities, 15(8), 995-1016.

Cavalcante, A. (2016). "I did it all online:" Transgender identity and the management of everyday life. Critical Studies in Media Communication, 33, 109-122.

Chaney, C., \& Robertson, R. V. (2013). Racism and police brutality in America. Journal of African American Studies, 17, 480-505.

Cheng, C. Y., \& Lee, F. (2009). Multiracial identity integration: Perceptions of conflict and distance among multiracial individuals. Journal of Social Issues, 65, 51-68.

Choudhury, P. P., Badhan, N. S., Chand, J., Chhugani, S., Choskey, R., Husainy, S., ... Wat, E. C. (2009). Community alienation and its impact on help-seeking behavior among LGBTIQ South Asians in Southern California. Journal of Gay \& Lesbian Social Services, 21, 247-266.

Chun, C. A., Moos, R. H., \& Cronkite, R. C. (2006). Culture: A fundamental context for the stress and coping paradigm. In Handbook of multicultural perspectives on stress and coping (pp. 29-53). Springer, Boston, MA. 
Chung, G., Oswald, R. F., \& Wiley, A. (2006). Good daughters: Three different ways of being Korean American queer women. Journal of GLBT Family Studies, 2, 101124.

Chung, R. H. G., Kim, B. S., \& Abreu, J. M. (2004). Asian American multidimensional acculturation scale: Development, factor analysis, reliability, and validity. Cultural Diversity and Ethnic Minority Psychology, 10, 66.

Chung, Y. B., \& Katayama, M. (1998). Ethnic and sexual identity development of AsianAmerican lesbian and gay adolescents. Professional School Counseling, 1, 21-25.

Chung, Y. B., \& Szymanski, D. M. (2006). Racial and sexual identities of Asian American gay men. Journal of LGBT Issues in Counseling, 1, 67-93.

Chyten-Brennan, J. (2014). Sex and gender development. In L. Erickson-Schroth (Ed.) Trans Bodies, Trans Selves: A Resource for the Transgender Community, (pp. 265-290). New York City, New York: Oxford University Press.

Clements-Nolle, K., Marx, R., \& Katz, M. (2006). Attempted suicide among transgender persons: The influence of gender-based discrimination and victimization. Journal of homosexuality, 51, 53-69.

Collazo, A., Austin, A., \& Craig, S. L. (2013). Facilitating transition among transgender clients: Components of effective clinical practice. Clinical Social Work Journal, 41, 228-237.

Creswell, J. W. (2013). Qualitative inquiry and research design: Choosing among five approaches (3rd ed.). SAGE Publications, Inc.

Creswell, J. W., \& Miller, D. L. (2000). Determining validity in qualitative inquiry. Theory into Practice, 39, 124-130. 
Daley, A., Solomon, S., Newman, P. A., \& Mishna, F. (2007). Traversing the margins: Intersectionalities in the bullying of lesbian, gay, bisexual and transgender youth. Journal of Gay \& Lesbian Social Services, 19, 9-29.

Dang, A. A. T., \& Hu, M. (2005). Asian Pacific American Lesbian, gay, Bisexual, and Transgender People: A Community Portrait. National Gay and Lesbian Task Force Policy Institute.

Dargie, E., Blair, K. L., Pukall, C. F., \& Coyle, S. M. (2014). Somewhere under the rainbow: Exploring the identities and experiences of trans persons. The Canadian Journal of Human Sexuality, 23, 60-74.

Dasgupta, S. D. (1998). Gender roles and cultural continuity in the Asian Indian immigrant community in the US. Sex roles, 38, 953-974.

DeBlaere, C., Brewster, M. E., Sarkees, A., \& Moradi, B. (2010). Conducting research with LGB people of color: Methodological challenges and strategies.The Counseling Psychologist, 38, 331-362.

Decety, J., \& Sommerville, J. A. (2003). Shared representations between self and other: A social cognitive neuroscience view. Trends in Cognitive Sciences, 7, 527-533. Delgado-Romero, E. A., Galván, N., Maschino, P., \& Rowland, M. (2005). Race and ethnicity in empirical counseling and counseling psychology research a 10-year review. The Counseling Psychologist, 33, 419-448.

Deutsch, M. (2014). Sex and gender development. In L. Erickson-Schroth (Ed.) Trans Bodies, Trans Selves: A Resource for the Transgender Community, (pp. 241-264). New York City, New York: Oxford University Press.

Diamond, L. M., Pardo, S. T., \& Butterworth, M. R. (2011). Transgender experience and 
identity. In S. J. Schwartz, K. Luyckx, and V. L. Vignoles (Eds.) Handbook of identity theory and research (pp. 629-647). New York: Springer.

Dietert, M., \& Dentice, D. (2013). Growing up trans: socialization and the gender binary. Journal of GLBT Family Studies, 9, 24-42.

Eckhert, E. (2016). Focus: sex and gender health: A case for the demedicalization of queer bodies. The Yale Journal of Biology and Medicine, 89, 239-246.

Erich, S., Tittsworth, J., Dykes, J., \& Cabuses, C. (2008). Family Relationships and Their Correlations with Transsexual Well-Being. Journal of GLBT Family Studies, 4, 419-432.

Erich, S., Tittsworth, J., Meier, S. L. C., \& Lerman, T. (2010). Transsexuals of color: Perceptions of discrimination based on transsexual status and race/ethnicity status. Journal of GLBT Family Studies, 6, 294-314.

Erickson-Schroth, L., Gilbert, M. A., \& Smith, T. E. (2014). Sex and gender development. In L. Erickson-Schroth (Ed.) Trans Bodies, Trans Selves: A Resource for the Transgender Community, (pp. 80-101). New York City, New York: Oxford University Press.

Factor, R., \& Rothblum, E. (2008). Exploring gender identity and community among three groups of transgender individuals in the United States: MTFs, FTMs, and genderqueers. Health Sociology Review, 17, 235-253.

Fay, B. (1987). Critical social science. Ithaca, NY: Cornell University Press.

Festinger, L. (1954). A theory of social comparison processes. Human Relations, 7, 117140.

Fontaine, J. H. (2002). Transgender issues in counseling. In L. Burlew \& D. Capuzzi 
(Eds.), Sexuality counseling (pp. 177-194). New York: Nova Science Publishers, Inc.

Fuligni, A. J., Tseng, V., \& Lam, M. (1999). Attitudes toward family obligations among American adolescents with Asian, Latin American, and European backgrounds. Child development, 70, 1030-1044.

Giorgi, A. (2009). The descriptive phenomenological method in psychology: A modified Husserlian approach. Pittsburgh, PA: Duquesne University Press.

Glesne, C. (2015). Becoming qualitative researchers: An introduction (5th ed.). Boston: Pearson.

Goldman, B. M., \& Kernis, M. H. (2002). The role of authenticity in healthy psychological functioning and subjective well-being. Annals of the American Psychotherapy Association, 5(6), 18-20.

Goffman, E. (1955). On face-work: An analysis of ritual elements in social interaction. Psychiatry, 18, 213-231.

Graham, L. F. (2014). Navigating community institutions: Black transgender women's experiences in schools, the criminal justice system, and churches. Sexuality Research and Social Policy, 11, 274-287.

Grant, J.M., Mottet, L.A., Tanis, J., Harrison, J., Herman, J.L., \& Keisling, M. (2011). Injustice at every turn: National transgender discrimination survey. Washington, D.C.: National Center for Transgender Equality and National Gay and Lesbian Task Force.

Greene, B. (2000). Beyond heterosexism and across the cultural divide: Developing an inclusive lesbian, gay, and bisexual psychology A look into the future. In B. 
Greene \& G. L. Croom (Eds.), Education, research, and practice in lesbian, gay, bisexual, and transgendered psychology: A resource manual (pp. 1-45). Thousand Oaks, CA: Sage.

Grossman, A. H., \& D'augelli, A. R. (2006). Transgender youth: Invisible and vulnerable. Journal of homosexuality, 51, 111-128.

Grossman, A. H., D'Augelli, A. R., \& Frank, J. A. (2011). Aspects of psychological resilience among transgender youth. Journal of LGBT Youth, 8, 103-115.

Grossman, A. H., D'Augelli, A. R., Howell, T. J., \& Hubbard, S. (2005). Parents' reactions to transgender youths' gender nonconforming expression and identity. Journal of Gay \& Lesbian Social Services, 18, 3-16.

Grossman, A. H., D'Augelli, A. R., Salter, N. P., \& Hubbard, S. M. (2005). Comparing gender expression, gender nonconformity, and parents' responses of female-tomale and male-to-female transgender youth: Implications for counseling. Journal of LGBT Issues in Counseling, 1, 41-59.

Guba, E. G. (1981). Criteria for assessing the trustworthiness of naturalistic inquiries. Educational Communication and Technology, 29, 75-91.

Hamburger, C., Stürup, G. K., \& Dahl-Iversen, E. (1953). Transvestism: Hormonal, psychiatric, and surgical treatment. Journal of the American Medical Association, 152, 391-396.

Han, C. S. (2010). One gay Asian body: A personal narrative for examining human behavior in the social environment. Journal of Human Behavior in the Social Environment, 20, 74-87.

Harper, G. W., Jernewall, N., \& Zea, M. C. (2004). Giving voice to emerging science and 
theory for lesbian, gay, and bisexual people of color. Cultural Diversity and Ethnic Minority Psychology, 10, 187-199.

Hayes, J. A., Chun-Kennedy, C., Edens, A., \& Locke, B. D. (2011). Do double minority students face double jeopardy? Testing minority stress theory. Journal of College Counseling, 14, 117-126.

Hendricks, M. L., \& Testa, R. J. (2012). A conceptual framework for clinical work with transgender and gender nonconforming clients: An adaptation of the Minority Stress Model. Professional Psychology: Research and Practice, 43, 460-467.

Hitlin, S. (2003). Values as the core of personal identity: Drawing links between two theories of self. Social psychology quarterly, 66, 118-137.

Ho, D. Y. (1976). On the concept of face. American Journal of Sociology, 81, 867-884.

Ho, M. K., Rasheed, J. M., \& Rasheed, M. N. (2004). Family therapy with ethnic minorities (2nd ed.). Sage Publications.

Hoeffel, E. M., Rastogi, S., Kim, M. O., \& Hasan, S. (2012). The Asian population: 2010. US Department of Commerce, Economics and Statistics Administration, US Census Bureau.

Hotta, J., \& Ting-Toomey, S. (2013). Intercultural adjustment and friendship dialectics in international students: A qualitative study. International Journal of Intercultural Relations, 37, 550-566.

Hune, S. (2002). Demographics and diversity of Asian American college students. New directions for student services, 2002, 11-20.

Hwang, W. C., \& Ting, J. Y. (2008). Disaggregating the effects of acculturation and acculturative stress on the mental health of Asian Americans. Cultural Diversity 
and Ethnic Minority Psychology, 14, 147-154.

Ishikawa, M. E. (2013). Nisei, sansei, and yonsei: Acculturation, ethnic identity, and subjective well-being among three generations of Japanese Americans (Doctoral dissertation, WASHINGTON STATE UNIVERSITY).

Israel, G. E. (2004). Supporting transgender and sex reassignment issues: Couple and family dynamics. Journal of Couple \& Relationship Therapy, 3, 53-63.

Israel, G. E. (2005). Translove: Transgender persons and their families. Journal of GLBT Family Studies, 1, 53-67.

Iwamoto, D. K., Liao, L., \& Liu, W. M. (2010). Masculine norms, avoidant coping, Asian values, and depression among Asian American men. Psychology of men \& masculinity, 11, 15-24.

Jackson, N. C., Johnson, M. J., \& Roberts, R. (2008). The potential impact of discrimination fears of older gays, lesbians, bisexuals and transgender individuals living in small-to moderate-sized cities on long-term health care. Journal of Homosexuality, 54, 325-339.

James, S. E., Herman, J. L., Rankin, S., Keisling, M., Mottet, L., \& Anafi, M. (2016). The Report of the 2015 U.S. Transgender Survey. Washington, DC: National Center for Transgender Equality.

Jauk, D. (2013). Gender violence revisited: Lessons from violent victimization of transgender identified individuals. Sexualities, 16, 807-825.

Johnson, E. P. (2001). " Quare" studies, or (almost) everything I know about queer studies I learned from my grandmother. Text and Performance Quarterly, 21, 125. 
Jones, P. S., Lee, J. W., \& Zhang, X. E. (2011). Clarifying and measuring filial concepts across five cultural groups. Research in nursing \& health, 34, 310-326.

Kalra, G. (2012). Hijras: the unique transgender culture of India. International Journal of Culture and Mental Health, 5, 121-126.

Katz-Wise, S. L., Reisner, S. L., Hughto, J. M. W., \& Budge, S. L. (2017). Self-reported changes in attractions and social determinants of mental health in transgender adults. Archives of Sexual Behavior, 46, 1425-1439.

Kim, B. S. K., \& Abreu, J. M. (2001). Acculturation measurement: Theory, current instruments, and future directions. In J. G. Ponterotto, J. M. Casas, L. A. Suzuki, \& C. M. Alexander (Eds), Handbook of multicultural counseling (2nd ed., pp. 394-424). Thousand Oaks, CA: Sage.

Kim, B. S. K., Atkinson, D. R., \& Umemoto, D. (2001). Asian cultural values and the counseling process: Current knowledge and directions for future research. The Counseling Psychologist, 29, 570-603.

Kim, B. S. K., Atkinson, D. R., \& Yang, P. H. (1999). The Asian values scale: Development, factor analysis, validation, and reliability. Journal of Counseling Psychology, 46, 342-352.

Kim, B. S. K, \& Omizo, M. M. (2003). Asian cultural values, attitudes toward seeking professional psychological help, and willingness to see a counselor. The Counseling Psychologist, 31, 343-361.

Kim, B. S. K., \& Omizo, M. M. (2006). Behavioral acculturation and enculturation and psychological functioning among Asian American college students. Cultural Diversity and Ethnic Minority Psychology, 12, 245-258. 
Kim, B. S. K., Yang, P. H., Atkinson, D. R., Wolfe, M. M., \& Hong, S. (2001). Cultural values similarities and differences among Asian American ethnic groups. Cultural Diversity and Ethnic Minority Psychology, 7, 343-361.

Kim, H., \& Markus, H.R. (1999). Deviance or uniqueness, harmony or conformity? A cultural analysis. Journal Personality and Social Psychology, 77, 785-800.

Kim, J. Y., \& Nam, S. H. (1998). The concept and dynamics of face: Implications for organizational behavior in Asia. Organization Science, 9, 522-534.

Kim, S. Y., \& Wong, V. Y. (2002). Assessing Asian and Asian American parenting: A review of the literature. In K. Kurasaki, S. Okazaki, \& S. Sue (Eds.) Asian American mental health: Assessment methods and theories (pp. 185-219). Netherlands: Kluwer Academic Publishers.

Kincheloe, J. L., \& McLaren, P. (2002). Rethinking critical theory and qualitative research. In Y. Zou \& E. T. Trueba (Eds.), Ethnography and schools: Qualitative approaches to the study of education (pp. 87-138). Lanham, MD: Rowman \& Littlefield Publishers, Inc.

Koh, J. B. K., Shao, Y., \& Wang, Q. (2009). Father, mother and me: Parental value orientations and child self-identity in Asian American immigrants. Sex Roles, 60, 600-610.

Krafft-Ebing, R. V. (1892). Psychopathia sexualis, with especial reference to the contrary sexual instinct: A medico-legal study. Philadelphia, PA: F.A. Davis and Co.

Kuper, L. E., Nussbaum, R., \& Mustanski, B. (2012). Exploring the diversity of gender and sexual orientation identities in an online sample of transgender individuals. Journal of sex research, 49, 244-254. 
Kwak, K., \& Berry, J. W. (2001). Generational differences in acculturation among Asian families in Canada: A comparison of Vietnamese, Korean, and East-Indian groups. International journal of psychology, 36, 152-162.

LaFromboise, T., Coleman, H., \& Gerton, J. (1993). Psychological impact of biculturalism: Evidence and theory. Psychological Bulletin, 114, 395-412.

Lamble, S. (2008). Retelling racialized violence, remaking White innocence: The politics of interlocking oppressions in Transgender Day of Remembrance. Sexuality Research \& Social Policy, 5, 24-42.

Laurent, E. (2005). Sexuality and human rights: An Asian perspective. Journal of Homosexuality, 48, 163-225.

Lee, E. \& Mock, M. R. (2005). Asian families: An overview. In M. McGoldrick, J. Giordano, \& N. Garcia-Preto (Eds.), Ethnicity \& family therapy (3rd ed., pp. 269289). New York: The Guildford Press.

Lee, M. Y., \& Mjelde-Mossey, L. (2004). Cultural dissonance among generations: a solution-focused approach with East Asian elders and their families. Journal of Marital and Family Therapy, 30, 497-513.

Lee, R. M., Choe, J., Kim, G., \& Ngo, V. (2000). Construction of the Asian American family conflicts scale. Journal of Counseling Psychology, 47, 211-222.

Leong, F. T., \& Lau, A. S. (2001). Barriers to providing effective mental health services to Asian Americans. Mental health services research, 3, 201-214.

Levitt, H. M., \& Ippolito, M. R. (2014). Being transgender: The experience of transgender identity development. Journal of Homosexuality, 61, 1727-1758.

Liem, R., Lim, B. A., \& Liem, J. H. (2000). Acculturation and emotion among Asian 
Americans. Cultural Diversity and Ethnic Minority Psychology, 6, 13-31.

Lincoln, Y. S., \& Guba, E. G. (1985). Naturalistic inquiry. Newbury Park, California: Sage Publications.

Litt, A., Reich, T., Maymin, S., \& Shiv, B. (2011). Pressure and perverse flights to familiarity. Psychological Science, 22, 523-531.

Liu, J. H., Ng, S. H., Weatherall, A., \& Loong, C. (2000). Filial piety, acculturation, and intergenerational communication among New Zealand Chinese. Basic and Applied Social Psychology, 22, 213-223.

Lombardi, E. (2009). Varieties of transgender/transsexual lives and their relationship with transphobia. Journal of homosexuality, 56, 977-992.

Lum, T. Y., Yan, E. C., Ho, A. H., Shum, M. H., Wong, G. H., Lau, M. M., \& Wang, J. (2015). Measuring Filial Piety in the 21st Century Development, Factor Structure, and Reliability of the 10-Item Contemporary Filial Piety Scale. Journal of Applied Gerontology, 1-13.

Maguen, S., \& Shipherd, J. C. (2010). Suicide risk among transgender individuals. Psychology \& Sexuality, 1, 34-43.

Mak, W. W., \& Chen, S. X. (2006). Face concern: Its role on stress-distress relationships among Chinese Americans. Personality and Individual Differences, 41(1), 143153.

Mao, L., McCormick, J., de Ven, P. V. (2002). Ethnic and gay identification: gay Asian men dealing with divide. Culture, Health, \& Sexuality, 4, 419-430.

Marín, G. \& Gamba, R. J. (2003). Acculturation and changes in cultural values. In K. M. Chun, P. Balls Organista, \& G. Marin (Eds), Acculturation: Advances in theory, 
measurement, and applied research (pp. 83-93). Washington, D.C.: American Psychological Association.

Marksamer, J. (2008). And by the way, do you know he thinks he's a girl? The failures of law, policy and legal representation for transgender youth in juvenile delinquency courts. Sexuality Research \& Social Policy, 5, 72-92.

Markus, H. R., \& Kitayama, S. (1991). Culture and the self: Implications for cognition, emotion, and motivation. Psychological review, 98, 224.

Mathy, R. M. (2002). A nonclinical comparison of transgender identity and sexual orientation: A framework for multicultural competence. Journal of Psychology \& Human Sexuality, 13, 31-54.

McGuire, J. K., Anderson, C. R., Toomey, R. B., \& Russell, S. T. (2010). School climate for transgender youth: A mixed method investigation of student experiences and school responses. Journal of Youth and Adolescence, 39, 1175-1188.

McPhail, B. A. (2004). Questioning gender and sexuality binaries: What queer theorists, transgendered individuals, and sex researchers can teach social work. Journal of Gay \& Lesbian Social Services, 17, 3-21.

Meyer, I. H. (2003). Prejudice, social stress, and mental health in lesbian, gay, and bisexual populations: Conceptual issues and research evidence. Psychological Bulletin, 129, 674-697.

Meyer, I. H. (2010). Identity, stress, and resilience in lesbians, gay men, and bisexuals of color. The Counseling Psychologist, 38, 442-454.

Meyer, O., Dhindsa, M., \& Zane, N. (2011). Psychology of Asian American adults: Challenges and Strengths. In E. C. Chang \& C. A. Downey (Eds.), Handbook of 
race and development in mental health (pp. 169-187). New York: Springer.

Monro, S. (2000). Theorizing transgender diversity: Towards a social model of health. Sexual and Relationship Therapy, 15, 33-45.

Moody, C., Fuks, N., Peláez, S., \& Smith, N. G. (2015). “Without this, I would for sure already be dead": A qualitative inquiry regarding suicide protective factors among trans adults. Psychology of sexual orientation and gender diversity, 2, 266-280.

Moradi, B., DeBlaere, C., \& Huang, Y. P. (2010). Centralizing the experiences of LGB people of color in counseling psychology. The Counseling Psychologist, 38, 322330.

Moradi, B., Tebbe, E. A., Brewster, M. E., Budge, S. L., Lenzen, A., Ege, E.,...Flores, M. (2016). A content analysis of literature on trans people and issues: 2002-2012. The Counseling Psychologist, 1-32.

Morrow, R. D. (1987). Cultural differences: Be aware. Academic Therapy, 23, 143-149.

Morrow, S. L. (2005). Quality and trustworthiness in qualitative research in counseling psychology. Journal of Counseling Psychology, 52, 250-260.

Moscovici, S. (1984). The phenomenon of social representations. In Robert M. Farr und Serge Moscovici (Eds.): Social representations, (pp. 3-70). Cambridge, London, New York, New Rochelle.

Moustakas, C. (1994). Phenomenological research methods. Thousand Oaks, CA: Sage. Mui, A. C., \& Kang, S. Y. (2006). Acculturation stress and depression among Asian immigrant elders. Social Work, 51, 243-255.

Nadal, K. L., \& Corpus, M. J. (2013). “Tomboys” and "Baklas": Experiences of lesbian and gay Filipino Americans. Asian American Journal of Psychology, 4, 166-175. 
Nadal, K. L., Davidoff, K. C., Davis, L. S., Wong, Y., Marshall, D., \& McKenzie, V. (2015). A qualitative approach to intersectional microaggressions: understanding influences of race, ethnicity, gender, sexuality, and religion. Qualitative Psychology, 2, 147-163.

Nadal, K. L., Skolnik, A., \& Wong, Y. (2012). Interpersonal and systemic microaggressions toward transgender people: Implications for counseling. Journal of LGBT Issues in Counseling, 6, 55-82.

National Queer Asian Pacific Islander. (2011). Injustice at every turn: A look at Asian American, South Asian, Southeast Asian, and Pacific Islander respondents in the national transgender discrimination survey. Washington, D.C.: National Center for Transgender Equality and National Gay and Lesbian Task Force.

Nemoto, T., Operario, D., Keatley, J., Han, L., \& Soma, T. (2004). HIV risk behaviors among male-to-female transgender persons of color in San Francisco. American Journal of Public Health, 94, 1193-1199.

Nicolazzo, Z. (2016). 'It's a hard line to walk': black non-binary trans* collegians' perspectives on passing, realness, and trans*-normativity. International Journal of Qualitative Studies in Education, 1-16.

Norwood, K. (2012). Transitioning meanings? Family members' communicative struggles surrounding transgender identity. Journal of Family Communication, 12, 75-92.

Ohbuchi, K. I., Fukushima, O., \& Tedeschi, J. T. (1999). Cultural values in conflict management goal orientation, goal attainment, and tactical decision. Journal of Cross Cultural Psychology, 30, 51-71. 
Ohbuchi, K. I., \& Takahashi, Y. (1994). Cultural Styles of Conflict Management in Japanese and Americans: Passivity, Covertness, and Effectiveness of Strategies1. Journal of Applied Social Psychology, 24, 1345-1366.

Ohnishi, H., Ibrahim, F. A., \& Grzegorek, J. L. (2007). Intersections of identities: Counseling lesbian, gay, bisexual, and transgender Asian-Americans. Journal of LGBT Issues in Counseling, 1, 77-94.

Okazaki, S. (2002). Influences of culture on Asian Americans' sexuality. Journal of sex research, 39, 34-41.

Park, M., \& Chesla, C. (2007). Revisiting Confucianism as a conceptual framework for Asian family study. Journal of Family Nursing, 13, 293-311.

Park, Y. S., Kim, B. S., Chiang, J., \& Ju, C. M. (2010). Acculturation, enculturation, parental adherence to Asian cultural values, parenting styles, and family conflict among Asian American college students. Asian American Journal of Psychology, 1, 67-79.

Poasa, K. (1992). The Samoan fa'afafine: One case study and discussion of transsexualism. Journal of psychology \& human sexuality, 5, 39-51.

Pollock, L., \& Eyre, S. L. (2012). Growth into manhood: Identity development among female-to-male transgender youth. Culture, health \& sexuality, 14, 209-222.

Poteat, T., German, D., \& Kerrigan, D. (2013). Managing uncertainty: a grounded theory of stigma in transgender health care encounters. Social Science \& Medicine, 84, 22-29.

Pyke, K. (2005). "Generational deserters" and "black sheep" acculturative differences among siblings in Asian immigrant families. Journal of Family Issues, 26, 491- 
517.

Rateau, P., Moliner, P., Guimelli, C., \& Abric, J. C. (2011). Social representation theory. Handbook of Theories of Social Psychology, 2, 477-497.

Reber, R., Winkielman, P., \& Schwarz, N. (1998). Effects of perceptual fluency on affective judgments. Psychological Science, 9, 45-48.

Redfield, R., Linton, R., \& Herskovits, M. (1936). Memorandum on the study of acculturation. American Anthropologist, 38, 149-152.

Reisner, S. L., White Hughto, J. M., Gamarel, K. E., Keuroghlian, A. S., Mizock, L., \& Pachankis, J. E. (2016). Discriminatory Experiences Associated With Posttraumatic Stress Disorder Symptoms Among Transgender Adults. Journal of Counseling Psychology, 1-11.

Reynolds, H. M. \& Goldstein, Z. G. (2014). Sex and gender development. In L. EricksonSchroth (Ed.) Trans Bodies, Trans Selves: A Resource for the Transgender Community, (pp. 124-154). New York City, New York: Oxford University Press.

Riley, E. A., Clemson, L., Sitharthan, G., \& Diamond, M. (2012). Counseling support for the forgotten transgender community. Journal of Gay \& Lesbian Social Services, $23,395-410$.

Riley, E. A., Clemson, L., Sitharthan, G., \& Diamond, M. (2013). Surviving a gendervariant childhood: The views of transgender adults on the needs of gender-variant children and their parents. Journal of sex \& marital therapy, 39, 241-263.

Roen, K. (2001). Transgender theory and embodiment: The risk of racial marginalisation. Journal of Gender Studies, 10, 253-263.

Romero, A. J., Carvajal, S. C., Valle, F., \& Orduña, M. (2007). Adolescent bicultural 
stress and its impact on mental well-being among Latinos, Asian Americans, and European Americans. Journal of Community Psychology, 35, 519-534.

Rosenthal, D., Ranieri, N., \& Klimidis, S. (1996). Vietnamese adolescents in Australia: Relationships between perceptions of self and parental values, intergenerational conflict, and gender dissatisfaction. International Journal of Psychology, 31, 8191.

Ross, K. A., \& Scholl, J. C. (2016). Socially supported transition: How transgender individuals use the internet to navigate medical transition. Iowa Journal of Communication, 48(1-2), 35-38.

Rosser, B. S., Oakes, J. M., Bockting, W. O., \& Miner, M. (2007). Capturing the social demographics of hidden sexual minorities: An Internet study of the transgender population in the United States. Sexuality Research \& Social Policy, 4, 50-64.

Rudmin, F. W. (2003). Critical history of the acculturation psychology of assimilation, separation, integration, and marginalization. Review of general psychology, 7, 337.

Ryder, A. G., Alden, L. E., \& Paulhus, D. L. (2000). Is acculturation unidimensional or bidimensional? A head-to-head comparison in the prediction of personality, selfidentity, and adjustment. Journal of personality and social psychology, 79, 49.

Salant, T., \& Lauderdale, D. S. (2003). Measuring culture: a critical review of acculturation and health in Asian immigrant populations. Social science \& medicine, 57, 71-90.

Sandelowski, M. (1995). Sample size in qualitative research. Research in nursing \& health, 18(2), 179-183. 
Schilt, K. (2006). Just one of the guys? How transmen make gender visible at work. Gender \& Society, 20, 465-490.

Schwartz, S. H. (1992). Universals in the content and structure of values: Theoretical advances and empirical tests in 20 countries. Advances in experimental social psychology, 25, 1-65.

Schwartz, S. H. (1994). Are there universal aspects in the structure and contents of human values? Journal of social issues, 50, 19-45.

Shenton, A. K. (2004). Strategies for ensuring trustworthiness in qualitative research projects. Education for Information, 22, 63-75.

Shim, Y. R., \& Schwartz, R. C. (2008). Degree of acculturation and adherence to Asian values as correlates of psychological distress among Korean immigrants. Journal of Mental Health, 17, 607-617.

Shin, K. R. (1994). Psychosocial predictors of depressive symptoms in Korean-American women in New York City. Women \& Health, 21, 73-82.

Shon, S. P., \& Ja, D. Y. (1982). Asian families. In M. McGoldrick, J. K. Pearce, \& J. Giordano (Eds.). Ethnicity and family therapy (pp. 208-228). New York: The Guildford Press.

Singelis, T. M., Triandis, H. C., Bhawuk, D. P. S., \& Gelfand, M. J. (1995). Horizontal and vertical dimensions of individualism and collectivism: A theoretical and measurement refinement. Cross-Cultural Resarch, 29, 240-275.

Singh, A. A. (2013). Transgender youth of color and resilience: Negotiating oppression and finding support. Sex Roles, 68, 690-702.

Singh, A. A., \& Burnes, T. R. (2010). Shifting the counselor role from gatekeeping to 
advocacy: Ten strategies for using the Competencies for Counseling with Transgender Clients for individual and social change. Journal of LGBT Issues in Counseling, 4, 241-255.

Singh, A. A., Hays, D. G., \& Watson, L. S. (2011). Strength in the face of adversity: Resilience strategies of transgender individuals. Journal of Counseling and Development, 89, 20-27.

Singh, A. A., \& McKleroy, V. S. (2011). "Just getting out of bed is a revolutionary act": The resilience of transgender people of color who have survived traumatic life events. Traumatology, 17, 34-44.

Singh, A. A., \& Shelton, K. (2011). A content analysis of LGBTQ qualitative research in counseling: A ten-year review. Journal of Counseling and Development, 89, 217226.

Stets, J. E., \& Burke, P. J. (2000). Identity theory and social identity theory. Social psychology quarterly, 63, 224-237.

Stets, J. E., \& Serpe, R. T. (2013). Identity theory. In Handbook of social psychology (pp. 31-60). Netherlands: Springer.

Stotzer, R. L. (2009). Violence against transgender people: A review of United States data. Aggression and Violent Behavior, 14, 170-179.

Stryker, S., \& Burke, P. J. (2000). The past, present, and future of an identity theory. Social psychology quarterly, 63, 284-297.

Su, D., Irwin, J. A., Fisher, C., Ramos, A., Kelley, M., Mendoza, D. A. R., \& Coleman, J. D. (2016). Mental Health Disparities Within the LGBT Population: A Comparison Between Transgender and Nontransgender Individuals. Transgender 
Health, 1(1), 12-20.

Sue, D., \& Sue, D. W. (1993). Ethnic identity: Cultural factors in the psychological development of Asians in America. In D. R. Atkinson, G. Morten, \& D.W. Sue (Eds.), Counseling American minorities: A cross-cultural perspective (4th ed., pp. 199-210). Dubuque, IA: W. C. Brown.

Sue, D. W., \& Sue, D. (2003). Barriers to effective multicultural counseling/therapy. In D. W. Sue \& D. Sue (Eds), Counseling the culturally diverse: Theory and practice (pp.95-121). New York: John Wiley and Sons.

Suinn, R. M., Rickard-Figueroa, K., Lew, S., \& Vigil, P. (1987). The Suinn-Lew Asian self-identity acculturation scale: An initial report. Educational and Psychological Measurement, 47, 401-407.

Sung, M. R., Szymanski, D. M., Henrichs-Beck, C. (2015). Challenges, coping, and benefits of being an Asian American lesbian or bisexual women. Psychology of Sexual Orientation and Gender Diversity, 2, 52-64.

Szapocznik, J., Scopetta, M. A., Kurtines, W., \& Aranalde, M. D. (1978). Theory and measurement of acculturation. Interamerican Journal of Psychology, 12, 113-130.

Szymanski, D. M., \& Sung, M. R. (2010). Minority stress and psychological distress among Asian American sexual minority persons. The Counseling Psychologist, 38, 848-872.

Tang, T. N., \& Dion, K. L. (1999). Gender and acculturation in relation to traditionalism: Perceptions of self and parents among Chinese students. Sex Roles, 41, 17-29.

Taylor, E. (2010). Cisgender privilege: On the privileges of performing normative gender. In K. Bornstein \& S. B. Bergman (Eds.), Gender outlaws: The next 
generation, (pp. 268-272). Berkeley, California: Seal Press.

Testa, R. J., Sciacca, L. M., Wang, F., Hendricks, M. L., Goldblum, P., Bradford, J., \& Bongar, B. (2012). Effects of violence on transgender people. Professional Psychology: Research and Practice, 43, 452.

Ting-Toomey, S. (1994). Face and facework: An introduction. In S. Ting-Toomey (Ed.). The challenge of facework: Cross-cultural and interpersonal issues (pp. 114). Albany, NY: State University of New York Press.

Ting-Toomey, S. (2005). Identity negotiation theory: Crossing cultural boundaries. In W. B. Gudykunst (Ed.), Theorizing about intercultural communication (pp. 211-233). Thousand Oaks, CA: Sage.

Transgender Law Center. (2016). National equality map. Retrieved from http://transgenderlawcenter.org/equalitymap

Tremble, B., Schneider, M., \& Appathurai, C. (1989). Growing up gay or lesbian in a multicultural context. Journal of Homosexuality, 17, 253-267.

Triandis, H. C. (2001). Individualism-collectivism and personality. Journal of Personality, 69, 907-924.

Triandis, H. C., McCusker, C., \& Hui, C. H. (1990). Multimethod probes of individualism and collectivism. Journal of Personality and Social Psychology, 59, 1006-1020.

Trotter II, R. T. (2012). Qualitative research sample design and sample size: Resolving and unresolved issues and inferential imperatives. Preventive Medicine, 55, 398400.

Trubisky, P., Ting-Toomey, S., \& Lin, S. L. (1991). The influence of individualism- 
collectivism and self-monitoring on conflict styles. International journal of intercultural relations, 15, 65-84.

Tsai-Chae, A. H., \& Nagata, D. K. (2008). Asian values and perceptions of intergenerational family conflict among Asian American students. Cultural Diversity and Ethnic Minority Psychology, 14, 205-214.

Tuli, M., \& Chaudhary, N. (2010). Elective interdependence: Understanding individual agency and interpersonal relationships in Indian families. Culture \& Psychology, 16, 477-496.

Ungar, M. (2005). Pathways to resilience among children in child welfare, corrections, mental health and educational settings: Navigation and negotiation. Child and Youth Care Forum, 34, 423-444.

VanderLaan, D. P., Petterson, L. J., Mallard, R. W., \& Vasey, P. L. (2015). (Trans) gender role expectations and child care in Samoa. The Journal of Sex Research, 52, 710-720.

Vera, E. M., \& Speight, S. L. (2003). Multicultural competence, social justice, and counseling psychology: Expanding our roles. The Counseling Psychologist, 31, 253-272.

Vivero, V. N., \& Jenkins, S. R. (1999). Existential hazards of the multicultural individual: Defining and understanding" cultural homelessness.". Cultural Diversity and Ethnic Minority Psychology, 5, 6-25.

Wertz, F. J. (2005). Phenomenological research methods for counseling psychology. Journal of counseling psychology, 52, 167.

White, T., \& Ettner, R. (2004). Disclosure, risks and protective factors for children whose 
parents are undergoing a gender transition. Journal of Gay \& Lesbian Psychotherapy, 8, 129-145.

Wight, J. (2011). Facing gender performativity: How transgender performances and performativity trouble facework research. Kaleidoscope: A Graduate Journal of Qualitative Communication Research, 10, 6 .

Winter, S. (2006). Thai transgenders in focus: Demographics, transitions and identities. International Journal of Transgenderism, 9, 15-27.

Winter, S., Rogando-Sasot, S., \& King, M. (2007). Transgendered women of the Philippines. International Journal of Transgenderism, 10, 79-90.

Witten, T. M., Benestad, E. E. P., Berger, I., Ekins, R., Ettner, R., Harima, K., ... \& Sharpe, A. N. (2003). Transgender and transsexuality. In Encyclopedia of Sex and Gender (pp. 216-229). Springer US.

Wong, F. Y., Chng, C. L., Ross, M. W., \& Mayer, K. H. (1998). Sexualities as social roles among Asian-and Pacific Islander American gay, lesbian, bisexual, and transgender individuals: Implications for community-based health education and prevention. Journal of the Gay and Lesbian Medical Association, 2, 157-166.

Wooden, W. S., Kawasaki, H., \& Mayeda, R. (1983). Lifestyles and identity maintenance among gay Japanese-American males. Alternative Lifestyles, 5, 236-243.

Xavier, J. M., Bobbin, M., Singer, B., \& Budd, E. (2005). A needs assessment of transgendered people of color living in Washington, DC. International Journal of Transgenderism, 8, 31-47.

Xia, Y. R., Do, K. A., \& Xie, X. (2013). The adjustment of Asian American families to the US context: The ecology of strengths and stress. In G. W. Peterson \& K. R. 
Bush (Eds.) Handbook of Marriage and the Family (3rd ed., pp. 705-722). Springer US.

Yee, B. W. K., DeBaryshe, B. D., Yuen, S., Kim, S. Y., \& McCubbin, H. I. (2007). Asian American and Pacific Islander families: Resiliency and life-span socialization in a cultural context. In F. T. L. Leong et al. (Eds), Handbook of Asian American Psychology (pp. 379-405). Thoadsand Oaks, CA: Sage.

Zajonc, R. B. (1968). Attitudinal effects of mere exposure. Journal of Personality and Social Psychology, 9(2, Pt.2), 1-27.

Zea, M. C., Reisen, C. A., \& Díaz, R. M. (2003). Methodological issues in research on sexual behavior with Latino gay and bisexual men. American journal of community psychology, 31, 281-291.

Zhang, N., \& Dixon, D. N. (2003). Acculturation and attitudes of Asian international students toward seeking psychological help. Journal of Multicultural Counseling and Development, 31, 205-223. 


\section{APPENDIX A}

Demographic Questionnaire

1. What is your current gender identity?

a. Male/Man/Transmale/Transman

b. Female/Woman/Transfemale/Transwoman

c. Gender Nonbinary (e.g., Genderqueer, Genderfluid, etc.)

2. Please indicate any or all label(s) you use to describe your gender identity.

3. How old were you when you first started understanding your trans identity?

4. How old were you when you began identifying as trans?

5. What is your sex assigned at birth?
a. Male
b. Female
c. Intersex

6. Please indicate any or all label(s) you currently use to describe your sexual identity.

7. Please indicate any or all label(s) you use to describe your gender expression. (e.g., femme, masculine, androgynous, stud, etc.)

8. What is your age?

9. What is your ethnicity and/or nationality? Please list all that apply. (e.g., Chinese, Japanese American, Korean American, Hmong, Hawaiian, Samoan, etc.)

10. What generation Asian, Asian American, and/or Pacific Islander are you? (i.e., First Generation = immigrated as an adult; 1.5 Generation $=$ immigrated as a child; Second Generation = born in the U.S. to immigrant parents; etc.) 
a. If First or 1.5 Generation, please indicate (in years) how long since you have been in the U.S.:

b. If Second Generation, please indicate (in years) how long your parent(s) have been in the U.S.:

11. In miles, roughly estimate how far away does your family lives from you:

12. From a range of 0 to 100 , where do you believe you are in your identity (or transition) process, with 0 being "I haven't begun" to 100 being "I've reached the furthest point I can in my process." Please circle your response:

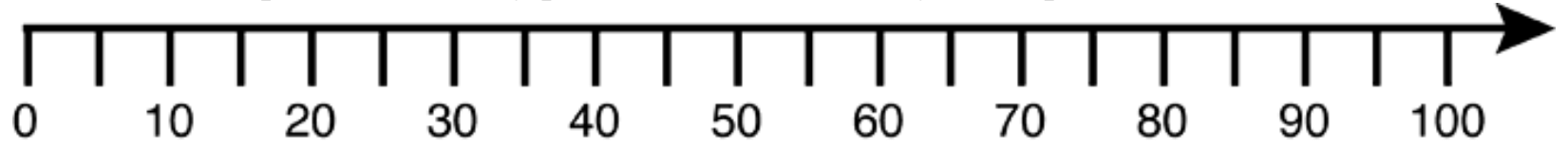

a. Please briefly describe why you chose the number above.

13. Where do you live? Please indicate city, state, and zip code.

14. Would you define where you live as urban, rural, or suburban?

15. Please indicate your highest level of completed education:
a. Did not complete high school
b. High school diploma/GED
c. Some college
d. Associates Degree
e. Bachelors Degree
f. Masters Degree
g. Doctoral/Professional Degree

16. Please indicate your current individual yearly income:
a. $\$ 0-\$ 10,000$
b. $\$ 10,001-\$ 20,000$
c. $\$ 20,001-\$ 30,000$ 

d. $\$ 40,001-\$ 50,000$
e. $\$ 50,001-\$ 60,000$
f. $\$ 60,001-\$ 70,000$
g. $\$ 70,001-\$ 80,000$
h. $\$ 80,001-\$ 90,000$
i. $\$ 90,001-\$ 100,000$
j. \$100,001 and above 


\section{APPENDIX B}

Interview Protocol

1. How do you identify in terms of your gender identity? What are your pronouns? How long have you identified as trans?

a. What does it mean to you to be [gender identity]?

2. How do you identify in terms of your race and ethnicity? What generation are you?

a. Who is in your family?

b. What does it mean to you to be [ethnicity]?

3. Are you out to your family and [ethnicity] community about your trans identity?

a. Yes: Please describe your coming out experience with your family and [ethnicity] community? How has coming out to your family and [ethnicity] community as trans influenced your relationships with them?

b. No: Please describe your decision to not come out to your family and [ethnicity] community? How has not coming out to them influenced your relationships with them?

4. Describe the cultural values that were instilled into you by your family.

a. How do you perceive these values influenced your [ethnicity] identity? Trans identity?

5. Describe the cultural values that were instilled into you by mainstream American society.

a. How do you perceive these values influenced your trans identity? [Ethnicity] identity?

6. Describe your experiences being a trans [ethnicity] person in the trans community and in trans spaces.

7. Describe your experiences being a trans [ethnicity] person in your family and in [ethnic identity] spaces.

8. Describe times when you had to negotiate your [ethnicity] and mainstream American cultural values for your trans [ethnicity] identities.

a. What helped the negotiation process?

b. What things do you wish had been in place to make this process easier?

c. How do you think this process affected your emotional and/or psychological well-being, if at all?

9. What do you wish the [ethnicity] community knew about you? What do you wish the trans community knew about you?

10. What does being a trans [ethnicity] mean to you?

11. What would you like to see come out of this study? 


\title{
CURRICULUM VITA
}

Jayden Lee Thai, M.Ed.

\author{
Department of Education and Counseling Psychology \\ College of Education and Human Development \\ University of Louisville \\ Louisville, KY 40292 \\ jayden.thai13@gmail.com
}

\section{EDUCATION}

University of Louisville, Ph.D.

2018 (Anticipated)

APA-Accredited Counseling Psychology Program

Doctoral Candidate

Dissertation: Transgender and racial identities negotiation process of transgender Asians, Asian Americans, and Pacific Islanders: A transcendental phenomenological examination

University of Utah, University Counseling Center

2017-Present APA-Accredited, APPIC-listed Predoctoral Internship

University of Louisville, M.Ed.

APA-Accredited Counseling Psychology Program

University of Louisville, B.S.

2010

Major: Psychology

\section{PUBLICATIONS}

Puckett, J., Wadsworth, L., Barr, S. M., \& Thai, J. L. (In press). Considerations for clinical work and research with trans individuals. The Behavioral Therapist.

Budge, S. L. \& Thai, J. L. (2017). Coming out processes for transgender people. In K. Nadal (Ed.) The SAGE Encyclopedia of Psychology and Gender (pp. 357-360). Thousand Oaks, CA: SAGE.

Budge, S. L., Thai, J. L., Tebbe, E. N., \& Howard, K. H. (2016) The intersection of socioeconomic status, race, sexual orientation, transgender identity, and mental 
health outcomes. The Counseling Psychologist, 44, 1025-1049.

Sawyer, K. B., Thai, J. L., Martinez, L. R., Smith, N. A., \& Discont, S. (2016). Trans issues in the workplace 101. The Industrial-Organizational Psychologist.

Budge, S. L., Orovecz, J., \& Thai, J. L. (2015) Trans men and positivity: Emotional processes related to gender identity. The Counseling Psychologist, 43, 404-434.

\section{PUBLICATIONS IN REVISION AND UNDER REVIEW}

Thai, J. L. \& Budge, S. L. (In Revision). Suicidality and substance abuse in trans Asian and Pacific Islander Individuals. The Counseling Psychologist.

Barr, S. M., Thai, J. L., Rey, E. A., Adelson, J. L., Sharp, C., \& Keo-Meier, C. (Under Review). Direct effects of testosterone therapy on the mental health of transgender men continue over the first year of treatment. Transgender Health.

\section{MANUSCRIPTS IN PROGRESS}

Thai, J. L., Orovecz, J., \& Budge, S. L. (In progress). Negative emotional processes related to gender identity among trans men.

Thai, J. L., Dholakia, J., \& Snow, L. (In progress). Health care accessibility and barriers among trans individuals in Kentucky.

\section{PROFESSIONAL PRESENTATIONS}

Thai, J. L. (2018, August). Examination of Identity Negotiation Among Transgender Asians, Asian American, and Pacific Islanders. Poster accepted for the American Psychological Association Convention, San Francisco, California.

Bradford, N. J., Catalpa, J., Mackenzie, L., \& Thai, J. L. (2017, November). Applications of the master narrative framework to the transgender community. Symposium presented at the National Transgender Health Conference, Oakland, California.

Thai, J. L. (2017, April). Identity negotiation processes of transgender Asian, Asian Americans, and Pacific Islanders. Poster presented at the Great Lakes Conference, Muncie, Indiana.

Thai, J. L., Orovecz, J., \& Budge, S. L. (2017, January). "I was pretty sure I was doing the wrongest thing a wrong thing could be done": A qualitative examination of trans men's experiences of negative emotions. Presented at the United States 
Professional Association for Transgender Health Conference, Los Angeles, California.

Barr, S. M., Thai, J. L, \& Keo-Meier, C. (2017, January). Examining White and POC transgender men's non-linear decrease in depression, anxiety, and stress symptoms across the first year of testosterone treatment. Presented at the United States Professional Association for Transgender Health Conference, Los Angeles, California.

Rossman, K. \& Thai, J. L. (2016, March). Trans issues in psychiatric care. Presented at the Kentucky Psychiatric Medical Association Conference, Louisville, Kentucky.

Thai, J. L., From, A. E., \& Nash, B. (2015, November). Understanding and working with trans individuals in educational and therapeutic settings. Presented at the Kentucky Psychological Association Convention, Lexington, Kentucky.

Thai, J. L., Dholakia, J., \& Snow, L. (2015, June). Health care accessibility among trans individuals in Kentucky: A descriptive examination. Presented at the WPATH Graduate Student Research Symposium of the Philadelphia Trans Health Conference, Philadelphia, Pennsylvania.

Dholakia, J., Snow, L., Willenbrink, C., \& Thai, J. L. (2015, February). Trans health matters: A case study from Kentucky. Presented at the Take Root: Red State Perspectives on Reproductive Justice Conference, Norman, Oklahoma.

Thai, J. L. \& Budge, S. L. (2014, August). The impact of outness and family acceptance on outcomes among Asian Pacific Islander trans people. Presented at the American Psychological Association Conference, Washington, D.C.

Thai, J. L., Orovecz, J., \& Budge, S. L. (2014, August). Trans* men's experiences of positive emotions: An examination of gender identity and emotion labels. Presented at the American Psychological Association Conference, Washington, D.C.

Thai, J. L. \& Nguyen, Y. (2014, April). Trans ally 101: Be a better trans ally. Presented at the National Queer Asian Pacific Islander Alliance Southern Regional Conference, Atlanta, Georgia.

Thai, J. L. \& Budge, S. L. (2014, March). Outness, family acceptance, and mental health outcomes of Asian Pacific Islander transgender individuals. Presented the Division 44 Committee on Racial and Ethnic Diversity sponsored symposium at the Counseling Psychology Conference, Atlanta, Georgia.

Budge, S. L., Thai, J. L., Sinnard, M., Rossman, K., \& Orovecz, J. (2014, February). Developmental processes of positive emotions for trans* individuals: The interplay of interpersonal emotions and transition appraisal. Paper presented at 
the World Professional Association for Transgender Health Biannual Conference, Bangkok, Thailand.

Thai, J. L., Rossman, K., Orovecz, J., Garrison, K., \& Determann, R. (2013, June). Demystifying therapy: Guild to help trans* clients find the right therapist. Presented at the Philadelphia Trans Health Conference, Philadelphia, Pennsylvania.

Rossman, K., Thai, J. L., Stahl, A., Nguyen, Y., Sinnard, M. (2013, June). Navigating the social transition. Presented at the Philadelphia Trans Health Conference, Philadelphia, Pennsylvania.

Orovecz, J., Thai, J. L., \& Budge, S. L. (2013, April). I'm stoked about life: The emotional processes of trans men through a qualitative lens. Presented at the Spring Research Conference, Lexington, Kentucky.

Orovecz, J., Thai, J. L., \& Budge, S. L. (2013, March). “I'm me, and I'm proud to be me": A grounded theory analysis of trans men's emotional processes. Presented at the Kentucky Psychological Association Foundation Spring Academic Conference, Louisville, Kentucky.

Thai, J. L. \& Rossman, K. (2013, March). Advocating for yourself: Self-care skills building for those in the LGBT community. Presented at the Come Together Kentucky Conference, Berea, Kentucky.

Thai, J. L. (2013, March). Queer people of color. Presented at the Come Together Kentucky Conference, Berea, Kentucky.

Thai, J. L. (2013, March). Trans 101. Presented at the Come Together Kentucky Conference, Berea, Kentucky.

Thai, J. L. (2013, January). Where do we belong? A space for trans folks and their partners. Presented at the National Gay and Lesbian Task Force's Creating Change Conference, Atlanta, Georgia.

Thai, J. L., Orovecz, J., \& Budge, S. L. (2012, November). Trans men and positivity: Emotional processes related to gender identity. Paper presented at the Chicago LGBTQ Health and Wellness Conference, Chicago, Illinois.

Budge, S. L., Thai, J. L., Rossman, K. (2012, August). Intersecting identities and mental health outcomes for transsexual, cross-dressing, and genderqueer individuals. Poster presented at the Annual Meeting for the American Psychological Association, Orlando, Florida.

Budge, S. L., Rossman, K., \& Thai, J. L. (2012, May). "I just want to deal with it on my own": Learning helpful coping strategies related to LGBTQ stressors. Presented 
at the Come Together Kentucky Conference, Louisville, Kentucky.

Thai, J. L. \& Budge, S. L. (2012, April). The Intersection of SES, Race, Sexual

Orientation, Transgender Identity, and Mental Health Outcomes. Paper presented at the Spring Research Conference, Louisville, Kentucky.

\section{CLINICAL EXPERIENCE}

University Counseling Center, University of Utah

8/2017 - Present

APA-Accredited, APPIC-listed predoctoral internship

Supervisor: Karen Cone-Uemura, Ph.D.

- Provide individual, couple, and group therapy, crisis counseling, intake consultations, and outreach services to a diverse population of university students.

- Provide clinical supervision to doctoral-level practicum students, including reviewing and providing feedback on recorded session videos and case notes.

- Interdisciplinary collaboration with intake team to determine optimal services for new clients.

- Conduct full battery assessments.

- Co-facilitate an interpersonal therapy group for individuals living with mental health diagnoses.

- Co-facilitate a support and empowerment group for LGBTQ+ students.

- Co-facilitate psychoeducational workshops for a variety of topics, such as mindfulness and work-life balance.

- Engage in campus outreach activities and presentations.

- Member of UCC's (a) Intern Selection Committee and (b) Training Committee.

Indiana University Southeast, New Albany, IN $8 / 2016-4 / 2017$ Supervisor: Michael Day, Psy.D.

- Provide individual therapy to university students from various identities and life experiences.

- Ages range from 19-years-old to 56-years-old.

- Participated in various outreach events and workshops around campus.

Cardinal Success Program @ Nia Center, Louisville, KY Supervisor: Eugene Foster, Ed.D.

- Provided individual therapies to individuals of various ages and racial/ethnic identities. Ages ranged between 10-years-old to 63-years-old.

- Conducted one psychological evaluation on adolescent transgender individual in order to assess for appropriateness for hormonal therapy.

- Collaborated in writing psychological report and letters to physician for psychological appropriateness for medical interventions related to gender transition.

Spalding University CAPS Center, Louisville, KY

$8 / 2014-8 / 2016$

Supervisor: Allison From, Psy.D.

- Provide individual, couples, and group therapies to adult college students using various therapeutic interventions and theories including but not limited to, behavioral and cognitive modification, mindfulness/meditation exercises, progressive relaxation 
exercises, and motivational interviewing techniques.

- Lead weekly mindfulness/meditation groups.

- Co-developed an anxiety and stress management group.

- Participated in outreach events and opportunities around campus.

- Facilitated multiple transgender-related training to Spalding University's Psy.D. students

- Guest lectured for an undergraduate psychology course

Dennis Wagner, Ed.D., Private Practice, Louisville, Kentucky $\quad$ 1/2012 - 7/2012 Supervisor: Dennis Wagner, Ed.D.

- Administered psychological evaluations to adults and adolescents using a variety of assessment tools.

- Conduct clinical interviews, mini-mental health exams, and numerous psychometric assessment tests (e.g. WAIS-IV, WISC-IV, MMPI-2, PAI, IVA+Plus, MACI).

- Score psychometric assessment tests and write psychological assessment reports.

- Clients were referred to Dr. Wagner's office for diverse of reasons, such as filing for disabilities, ADHD evaluation, and diagnostic assessment. Based on results of the psychological evaluation, recommendations were made to facilitate and guide treatment.

- Conducted feedback sessions with clients to go over results of the psychological evaluation and further recommendations.

Communicare, Inc.,

$7 / 2011-12 / 2011$

Hardin County Main Clinic, Elizabethtown, Kentucky

Supervisor: Doug Olds, Psy.D.

- Provided outpatient individual, family, couples, and group psychotherapy to diverse population of adults, adolescents, and children at rural community mental health clinic in Elizabethtown, Kentucky.

- Worked with individuals of all ages.

- Clients were referred to clinic from the community due to low income.

- Individual, family, and couples therapy typically meet biweekly for 45 minutes to one hour. Conducted intake assessments, treatment plans, and treatment updates.

- Co-facilitated group therapy that meets once a week for two hours, during the entire duration of internship. Each group consisted of between five to twelve adult individuals of a variety of diagnosis, including but not limited to schizophrenia, bipolar disorder, depression, and anxiety.

- Led group therapy for new and non-compliant consumer with each group consisting between one to six adult individuals.

- Assisted clients in learning and utilizing coping skills, communication and interpersonal skills, and life skills among other skills and resources.

- Received one hour of individual supervision per week. Participated in weekly treatment team and staff meetings.

Communicare, Inc., Hardin County Clinic, Radcliff, Kentucky $5 / 2011-12 / 2011$ Supervisor: Doug Olds, Psy.D.

- Co-therapist providing outpatient group therapy to adult individuals of a variety of diagnosis (i.e. schizophrenia, depression, anxiety) in rural Radcliff, Kentucky.

- Co-facilitated group therapy that meets twice a week for two hours, during the entire duration of internship. Each group consisted of between five to seven adult individuals.

- Assisted group members in learning and utilizing a variety of coping skills and life skills (i.e. progressive relaxation techniques, cognitive reframing exercises, behavioral 
management).

- Led group therapy for new and non-compliant consumer with each group consisting between one to six adult individuals.

\section{WORKSHOPS/TRAINING PROVIDED}

2/2018 Facilitate 1.5-hour long training, entitled "Bleached: Beyond the whitewashed trans narrative", for the members of the LGBT-Affirmative Psychotherapist Guild of Utah.

8/2016 Facilitated 2-hour long Trans 101 training for the staff of Kentucky Youth Career Center in Louisville, Kentucky.

1/2016 Facilitated 3-hour long Trans 101 training for psychological nursing students at the University of Louisville.

11/2015 Co-facilitated LGBTQ cultural competency training for first year medical, nursing, dental, public health, and social work students at the University of Louisville.

9/2015 Facilitated a one and a half hour long Trans 101 for practicum students at the college counseling center at Spalding University.

3/2015 Co-facilitated a two hour long Trans 101 for Therapist training for the students and faculty of the Psy.D Clinical Psychology program of Spalding University, as well as the university's President, Vice-Present, Deans, Provosts, and athletic coaches, held at Spalding University.

3/2014 Co-facilitated an hour and a half long presentation for a graduate-level higher administration class on gender, sexuality, and trans identity, disparities, and allyship, held at the University of Louisville.

2/2014 Co-facilitated an hour-long training for University of Louisville's medical students and faculties on needs and challenges of, appropriate interpersonal interactions with, and how to advocate for their trans patients in the medical setting, held at the University of Louisville.

11/2013 Co-facilitated an hour and a half-long workshop for students, faculties, and community members to discuss the topic of the intersectionality between race and trans identity, held during Pride Week at the University of Louisville.

10/2013 Co-facilitated a two-hour workshop for the grassroots organization Kentucky Health Justice Network, on WPATH Standards of Care and the impact of the Affordable Care Act for transgender individuals.

9/2013 Assisted advisor in training University of Louisville graduate and 
undergraduate level students on how to code and analysis qualitative data using Charmaz (2006) Grounded Theory, held at the University of Louisville.

3/2013 Co-facilitated an hour-long training to students, staffs, faculties, and administrators of University of Louisville's College of Education on learning appropriate interactions with, unique needs and challenges of, and how to advocate for, transgender students and faculty, held at the University of Louisville.

12/2012 Co-facilitated an hour-and-a-half long workshop designed to empower LGBTQ-identified students at the University of Louisville to utilize effective self-care strategies over the winter break, held at the University of Louisville.

11/2012 Co-facilitated an hour-long training to students, staffs, faculties, and administrators of University of Louisville's College of Education on learning appropriate interactions with, unique needs and challenges of, and how to advocate for, transgender students and faculty, held at the University of Louisville.

10/2012 Facilitated six (6) fifteen-minute long workshop to individuals on learning adaptive coping strategies related to LGBTQ stressors, at the University of Louisville's Pride Week held at the University of Louisville.

\section{SUPERVISION EXPERIENCE}

\section{University of Utah}

8/2017 - Present

University Counseling Center

- Provide clinical supervision to two doctoral-level counseling psychology students.

- Review and provide feedback on recorded session videos and case notes.

- Facilitate supervisee's client conceptualization development, emphasizing multicultural considerations.

ECPY 629 -

Theories and Technique in Counseling and Psychotherapy

$1 / 2017-4 / 2017$ Laurie "Lali" D. McCubbin, Ph.D.

- As a teaching assistant for the course, assisted in supervising graduate/masters level students in their development of counseling and psychotherapy techniques.

\section{ECPY 755 - Supervision}

- Supervised a master's Counseling Psychology practicum student as part of a supervision training course. Met with the supervisee for six supervision sessions. Supervision sessions covered case management, skills development, and selfawareness. Reviewed recorded videos of sessions and received feedback on supervision performance from peers and supervisor. 
ECPY 629 -

Theories and Technique in Counseling and Psychotherapy

$1 / 2015-4 / 2015$

Sarah Kolb, Ph.D.

- As a teaching assistant, assisted in supervising graduate level students in their development of counseling techniques.

\section{MENTORSHIP EXPERIENCE}

Mentoring First Year Doctoral Student

$9 / 2014-8 / 2015$

University of Louisville

- Mentor a first year doctoral student in the Counseling Psychology program.

- Help the student transition into the program, answer questions the student had about the program and the community, help student process experiences, and provide support.

Mentoring Freshman Student

$9 / 2014-12 / 2014$

University of Louisville

- Mentored a LGBTQ freshman relating to life on campus as an LGBTQ student.

- Helped the student process experiences of college, find LGBTQ community, and utilize campus resources.

\section{KEYNOTE \& INVITED PRESENTATIONS}

Thai, J. L. (2015, November). Trans visibility in the media. Invited speaker at DePauw University, Greencastle, Indiana.

Thai, J. L. (2014, March). In Solidarity. Invited keynote speaker at the Asian Pacific Islander Issues Conference, Berkeley, California.

Thai, J. L. (2013, January). Queer AAPIs in the South. Invited panelist speaker at the Asian American Pacific Islander Institute at the National Gay and Lesbian Task Force's Creating Change Conference, Atlanta, Georgia.

\section{TEACHING EXPERIENCE}

\section{Teaching Assistantships}

ECPY 629

Theories and Techniques of Counseling and Psychotherapy

University of Louisville

Laurie "Lali" D. McCubbin, Ph.D.

- Supervised live and recorded mock therapy sessions; provided feedback for growth and development. 
- Developed lesson plans and facilitated class lectures, multicultural considerations and discussions, and interactive activities.

- Developed and graded quizzes, exams, and mock client conceptualization papers; provided feedback.

- Held office hours to meet with students and provide extra guidance.

ECPY 621

$1 / 2016-4 / 2016$

Differential Diagnosis

University of Louisville

Laurie "Lali" D. McCubbin, Ph.D.

- Developed lesson plans and facilitated class lectures, multicultural considerations and discussions, and interactive activities.

- Developed and graded quizzes, exams, and mock client diagnostic conceptualization and treatment papers; provided feedback.

\section{ECPY 629}

Theories and Techniques in Counseling and Psychotherapy

University of Louisville

Sarah Kolb, Ph.D.

- Supervised live and recorded mock therapy sessions, provided feedback for growth and development.

- Developed lesson plans and facilitated class lectures and discussions.

- Graded mock client conceptualization papers.

ECPY 663

$5 / 2014-5 / 2014$

\section{Multicultural and Diversity Issues}

University of Louisville

Stephanie L. Budge, Ph.D.

- Developed lesson plans and facilitated class lectures and discussions related to multiculturalism in the counseling context.

- Graded and provided weekly feedback regarding class assignments and reflection papers.

\section{Invited Guest Lectures}

ECPY 663 - Multicultural and Diversity Issues

$6 / 2016$

University of Louisville

Ahmad Washington, Ph.D.

ECPY 621 - Differential Diagnosis

$4 / 2016$

University of Louisville

Katy Hopkins, Ph.D.

Multicultural Class

$7 / 2015$

Spalding University, Psy.D. Program

Norah Chapman, Psy.D. 
WGST 491 - Transgender Studies

University of Louisville

Ryan Combs, Ph.D.

WGST 201 - Women in American Culture

$12 / 2014$

University of Louisville

Yasmeen Chism, B.A.

ECPY 629 - Differential Diagnosis

$11 / 2014$

University of Louisville

Katy Hopkins, Ph.D.

Careers in Psychology Class

$10 / 2014$

Spalding University, Undergraduate

Barbara Beauchamp, Psy.D.

Cultural Appreciation Class

$6 / 2014$

National College

Yolanda Williams, M.Ed.

\section{DEPARTMENTAL SERVICE WORK}

Training Committee, University of Utah Counseling Center

$8 / 2017-4 / 2018$

Intern Selection Committee, University of Utah Counseling Center $\quad 11 / 2017-2 / 2018$

Doctoral Student Representative, CPY

$9 / 2016-7 / 2017$

Interviewer, Masters Student Search Committee for CPY

$2 / 2015,3 / 2016$

Interviewer, Doctoral Student Search Committee for CPY

2016

Member, Faculty Search Committee for CPY

$11 / 2014-3 / 2015$

Member, Doctoral Student Organization

$2013-2015$

\section{COMMUNITY/UNIVERSITY SERVICE WORK (Selected)}

Facilitator, Transformation

$9 / 2014-5 / 2016$

University of Louisville

Brian Buford, M.Ed.

- Facilitated a trans social/peer support group for trans students at the University of Louisville. 
Panelist, Inter-professional Solutions to LGBT Care

University of Louisville

Stacie Steinbock, M.Ed. and Chaz Briscoe, M.A.

- Participated as panelist on an inter-professional/interdisciplinary panel to discuss LGBT issues and care in various health care settings.

Panelist, Speak Out Team

$10 / 2012-6 / 2017$

University of Louisville

Katy Garrison, B.A.

- Participate as part of a panel of LGBTQ-identifying individuals, sharing personal stories related to identity development, intersecting identities, the coming out processes, social experiences, and interactions with medical and mental healthcare professionals, and answering questions from the audience. The audience varied, including Safe Zone trainings for students, faculty members, professors, staff, and administrators at the medical school, the medical school students and personnel, masters and doctoral level multicultural classes, sexual health classes, and communication classes at the University of Louisville. Participated in 19 Speak Out events so far.

Guest Blogger, Human Rights Campaign (HRC)

$11 / 2014$ Hyacinth Alvaran

- Invited to write a blog post in honor for Trans Week of Awareness for the Human Rights Campaign, a national LGBTQ rights advocacy organization.

- Blog was posted on HRC's multiple social media outlets and reached HRC's over two million followers.

Standardized Patient Training

$10 / 2014$

University of Louisville

Stacie Steinbock, M.Ed.

- Participated in UofL's LGBT Center's training of medical students on how to take inclusive, respectful, affirming intakes of LGBTQ-identified patients at their student-run medical clinic.

Member, Trans Week of Awareness Planning Committee

$9 / 2014$ Louisville, KY

- Collaborated with community leaders to develop and plan events during Trans Week of Awareness in the Louisville, KY.

Board Member, National Queer Asian Pacific Islander Alliance

$2 / 2014-3 / 2015$ Executive Directors: Ben de Guzman \& Glenn Magpantay

- Program Committee and Board Development Committee

- Collaboratively oversaw and assisted in the development and execution of NQAPIA programs and work with member groups, assist in defining and shaping the committee's new structure and goals so that it can more efficiently support the work of NQAPIA's board and staff, assist in the performance evaluation process for NQAPIA staff, develop board member applications, and recruit new board 
members.

Member, Conference Planning Committee

$1 / 2014-4 / 2014$

National Queer Asian Pacific Islander Alliance Southern Regional Conference

Executive Directors: Ben de Guzman \& Glenn Magpantay

- Helped plan, organize, and advertise the Southern Regional Conference for the National Queer Asian Pacific Islander Alliance, held on April 4-6 in Atlanta, Georgia.

- Specific responsibilities included participating in conference calls, contacting local agencies and community members about advertisement, registrations, and recruitment, and making decisions for speakers, location, housing, and programming.

Panelist, Intersections of Identities

University of Louisville

- Assisted in creating and conceptualizing the event, as part of Pride Week at the University of Louisville.

- Panelist and co-facilitator of the Pride Week event at the University of Louisville, talking about personal and community experiences of intersecting identities including but not limited to race/ethnic identity, gender identity, sexual identity, and geographic identity.

Introductory Speech for Keynote Speaker

$10 / 2013$

University of Louisville

Brian Buford, M.Ed. \& the LGBT Center

- Invited to give an introductory speech for the University of Louisville's Pride Week keynote speaker, Janet Mock - a well-known trans rights activist.

Judge, KPA Research Poster Presentation

University of Louisville

- Judged undergraduate research poster presentations as part of the Kentucky Psychological Association Student Research Conference.

\section{AWARDS AND SCHOLARSHIPS}

\section{Division 17 LGBTI Outstanding Graduate Student Award}

- Section of Lesbian, Gay, Bisexual, and Transgender Issues of APA Division 17 (Counseling Psychology) award to a graduate student in counseling psychology based on several criteria inclusive of scholarship, training, practice, service, and prevention/interventions.

Ethnic Minority Fellowship

$8 / 2013-6 / 2017$

Asian, Asian American, Pacific Islander Institute Scholarship 
$\begin{array}{lr}\text { Eric Rofes Memorial Scholarship } & 1 / 2013\end{array}$

$\begin{array}{ll}\text { NQAPIA Travel Scholarship Award } & \text { 7/2012 }\end{array}$

Come Together Kentucky Scholarship Award 3/2012

College of Education and Human

Development Scholarship Award

1/2011, 5/2011, $8 / 2011,1 / 2012$, $5 / 2012$

\section{MEMBERSHIP IN PROFESSIONAL ORGANIZATIONS}

American Psychological Association (APA)

Society of Counseling Psychology (Division 17)

Society for the Psychological Study of Lesbian, Gay, Bisexual, and Transgender Issues (Division 44)

American Psychological Association of Graduate Students (APAGS)

TSTAR (Trans and Sexuality Teaching, Advocacy, and Research) 